\title{
Eder Acquisition 2007 Habitat Evaluation Procedures Report
}

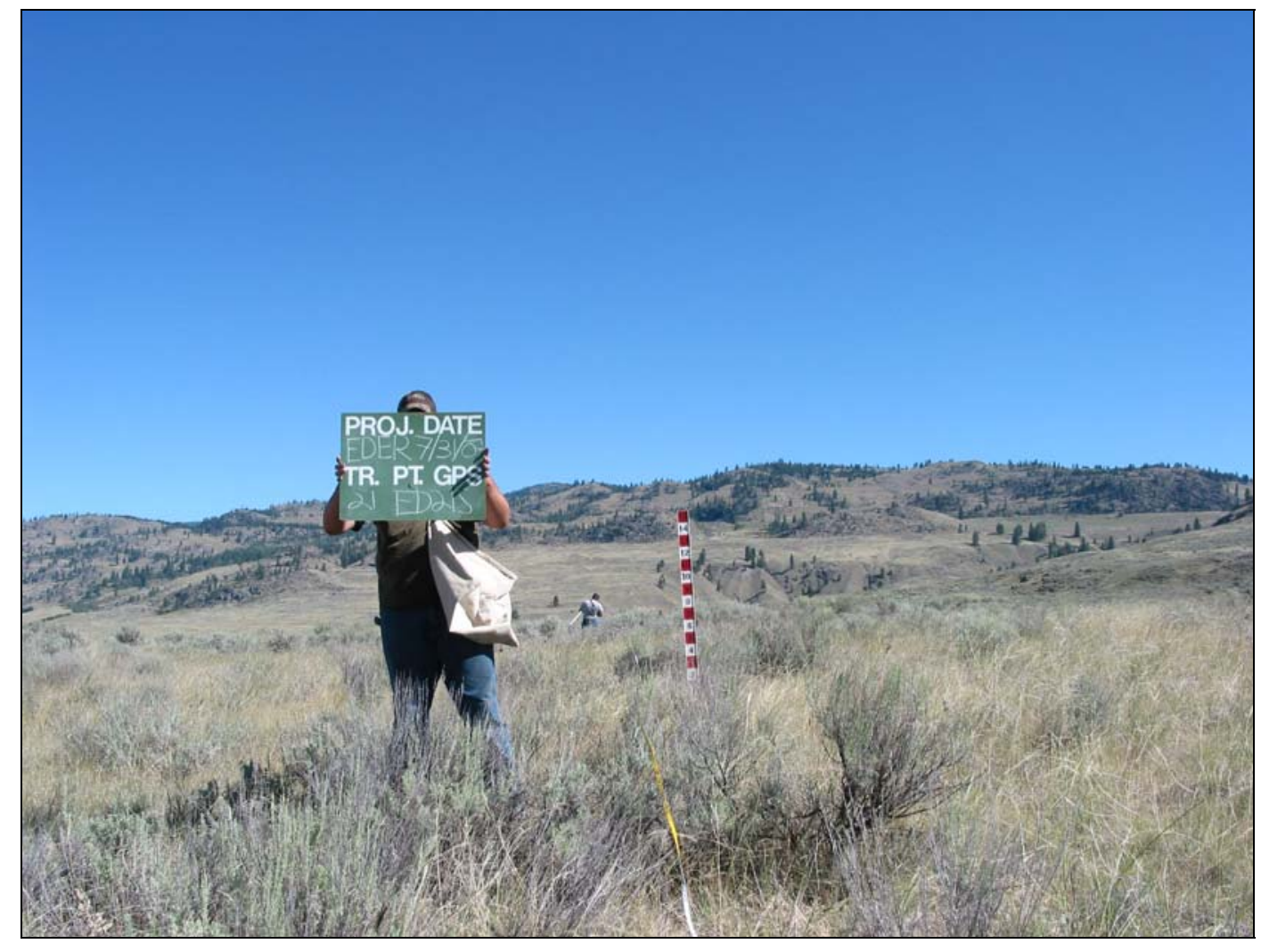

Compiled By

Paul R Ashley

Regional HEP Team Coordinator

For

Joe DeHerrera

Bonneville Power Administration

And

Nathan Pamplin

Washington Department of Fish and Wildlife

January 2008 


\section{Table of Contents}

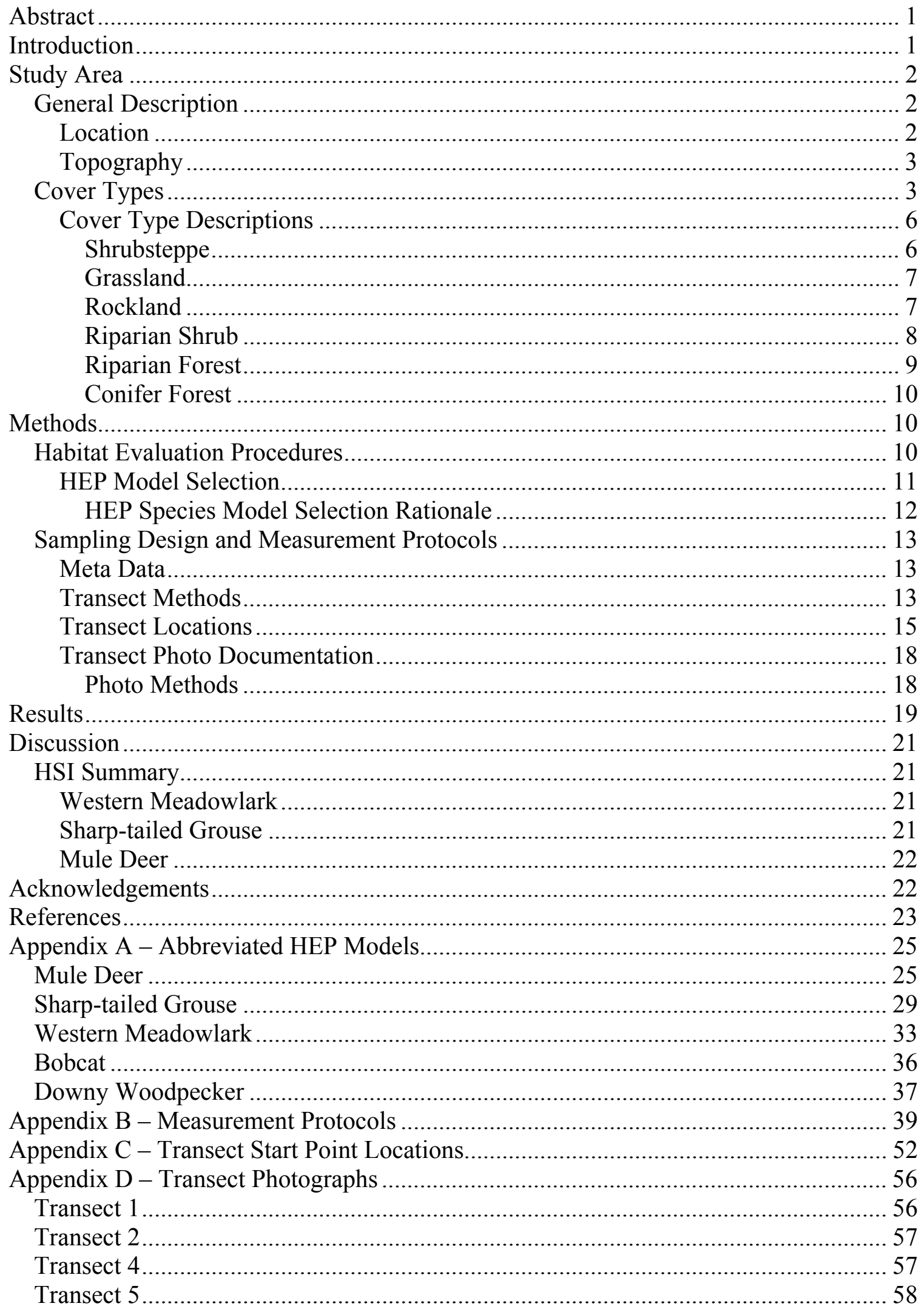


Eder Acquisition 2007 HEP Report

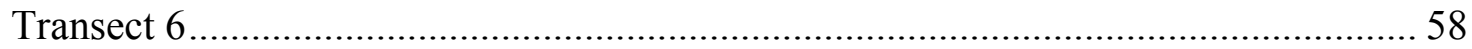

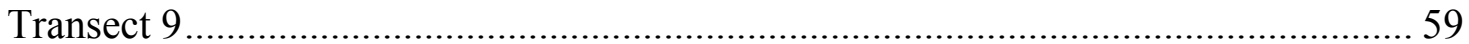

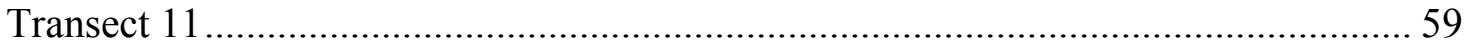

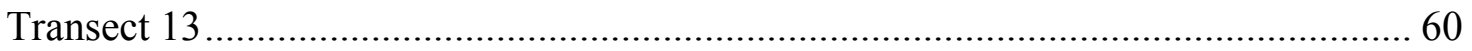

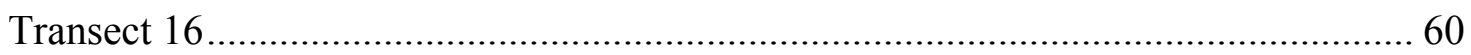

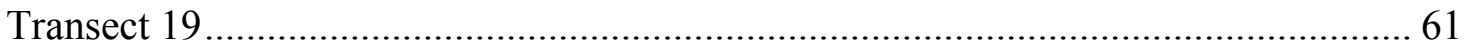

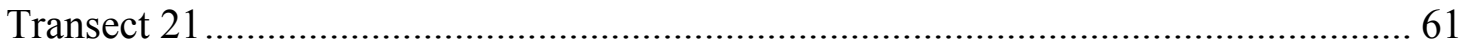

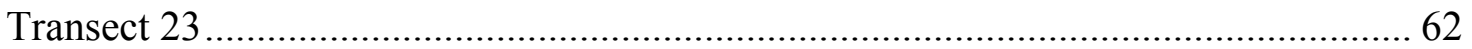

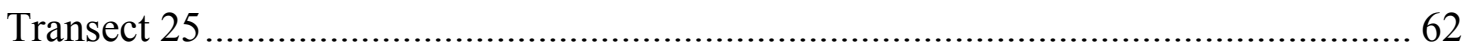

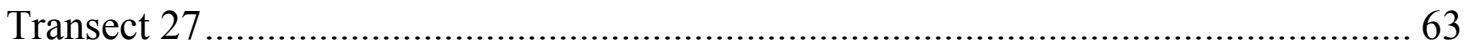

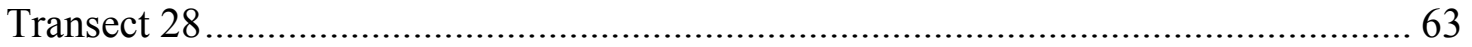

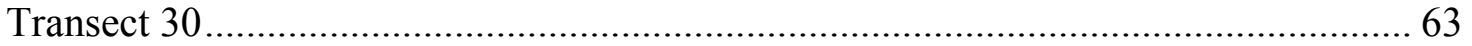

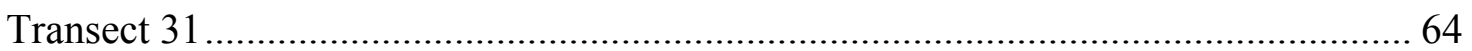

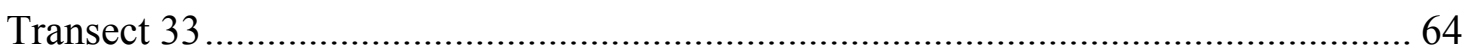

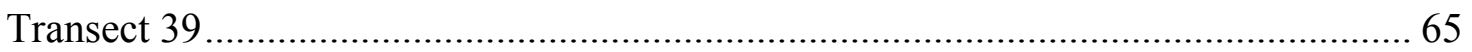

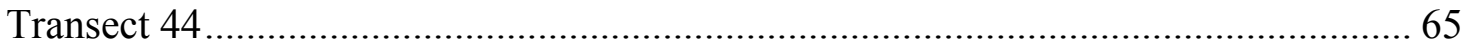

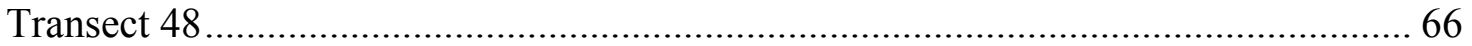

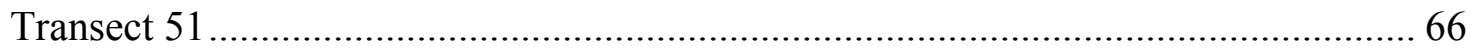

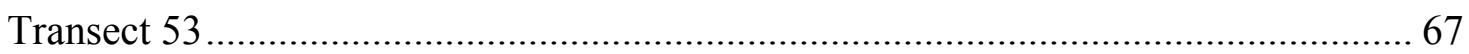

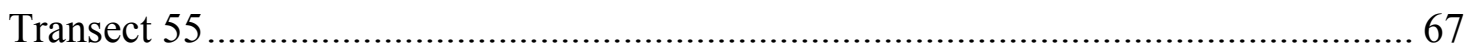

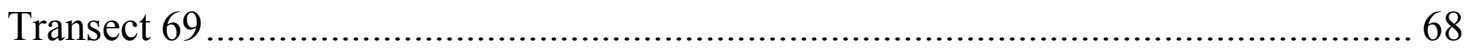


Eder Acquisition 2007 HEP Report

\section{List of Tables}

Table 1. Eder property cover types, acres, and relative percent of area. ............................ 4

Table 2. Habitat suitability index verbal equivalency table........................................... 11

Table 3. Eder project 2007 HEP loss assessment matrix................................................ 12

Table 4. HEP model species selection rationale table. ................................................... 12

Table 5. Eder HEP transect UTM coordinates, magnetic azimuths, and transect lengths. 17

Table 6. Eder acquisition 2007 HEP results summary. ................................................. 20 
Eder Acquisition 2007 HEP Report

\section{Table of Figures}

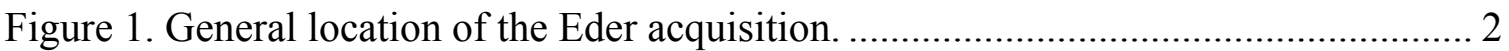

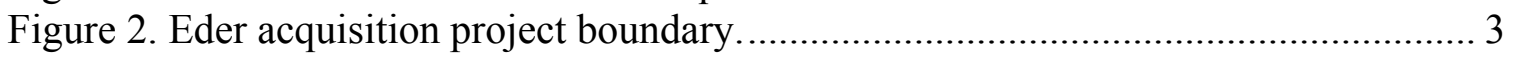

Figure 3. Eder property coarse filter cover type map. …………………...................... 5

Figure 4. An example of the shrubsteppe cover type....................................................... 6

Figure 5. An example of the grassland cover type...................................................... 7

Figure 6. An example of the rockland cover type......................................................... 8

Figure 7. An example of riparian shrub understory................................................ 9

Figure 8. Riparian forest cover type photo. ........................................................... 10

Figure 9. HEP data collection and processing flow chart.............................................. 14

Figure 10. Eder project transect initial points.......................................................... 15

Figure 11. Eder property 2007 HEP transect start points. ............................................ 16

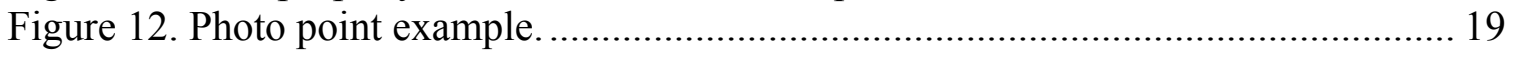




\section{Abstract}

A habitat evaluation procedures (HEP) analysis was conducted on the Eder acquisition in July 2007 to determine how many protection habitat units to credit Bonneville Power Administration (BPA) for providing funds to acquire the project site as partial mitigation for habitat losses associated with construction of Grand Coulee and Chief Joseph Dams. Baseline HEP surveys generated 3,857.64 habitat units or $1.16 \mathrm{HUs}$ per acre.

HEP surveys also served to document general habitat conditions. Survey results indicated that the herbaceous plant community lacked forbs species, which may be due to both livestock grazing and the late timing of the surveys. Moreover, the herbaceous plant community lacked structure based on lower than expected visual obstruction readings (VOR); likely a direct result of livestock impacts. In addition, introduced herbaceous vegetation including cultivated pasture grasses, e.g. crested wheatgrass and/or invader species such as cheatgrass and mustard, were present on most areas surveyed.

The shrub element within the shrubsteppe cover type was generally a mosaic of moderate to dense shrubby areas interspersed with open grassland communities while the "steppe" component was almost entirely devoid of shrubs. Riparian shrub and forest areas were somewhat stressed by livestock. Moreover, shrub and tree communities along the lower reaches of Nine Mile Creek suffered from lack of water due to the previous landowners "piping" water out of the stream channel.

\section{Introduction}

The Washington Department of Fish and Wildlife (WDFW) purchased the 3,337 acre Eder acquisition on June 28, 2007 (D. Budd and P. Dahmer, pers. comm.) in partnership with Bonneville Power Administration as partial fulfillment of BPA's mitigation obligation from construction of Grand Coulee Dam (Howerton et. al. 1986) and Chief Joseph Dam (Berger and Kuehn 1992). Both Memorandum of Agreement (MOA) (BPA/WDFW 1996) and Washington State Wildlife funds were used to purchase the property. MOA funds totaled $\$ 3,033,832$ while State expenditures equaled \$31,000 (P. Dahmer, pers. comm.). The 2007 acquisition included only the east portion of the Eder Ranch. WDFW intends to purchase the west half of the property in the near future (J. Olson, pers. comm.).

WDFW acquired the Eder Ranch primarily to protect critical winter deer range and sharp-tailed grouse (Typanuchus phasianellus columbianus) habitat (J. Olson, pers. comm.). A myriad of other wildlife species, however, will benefit from protection and enhancement measures.

A Habitat Evaluation Procedures (HEP) (USFWS 1980) analysis was conducted by the Columbia Basin Fish and Wildlife Authority's (CBFWA) Regional HEP Team (RHT) in 2007 to determine the number of habitat units (HUs) to credit BPA for providing the funds to acquire the property. Details and results of the HEP analysis are described in this report. 


\section{Study Area}

\section{General Description}

\section{Location}

Located in north central Washington approximately three miles northeast of Oroville, the Eder property borders Canada on the north and lies one mile east of Lake Osoyoos (Figure 1). Universal Trans Mercator (UTM) coordinates are 11U 0328200E, 5427310N.

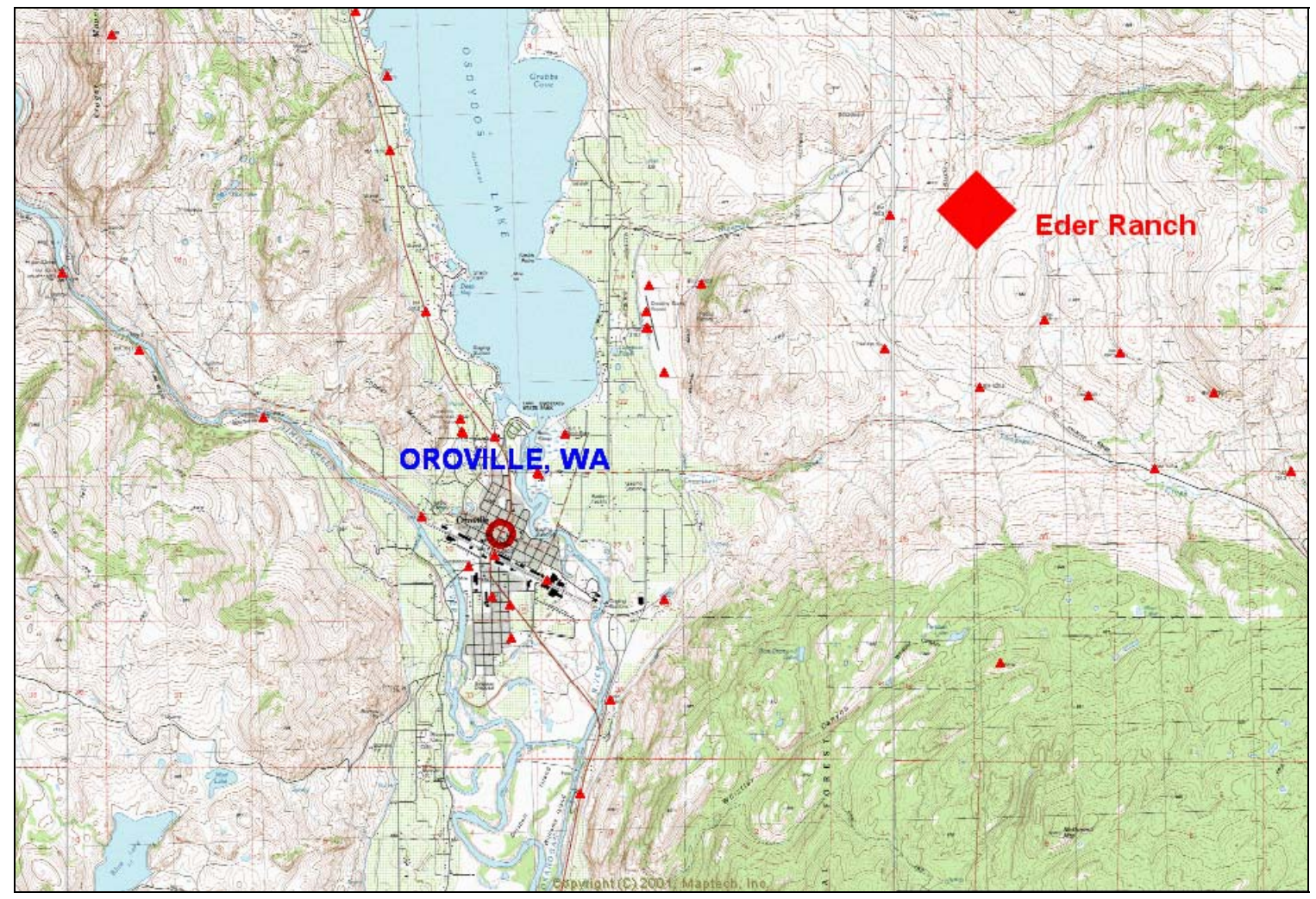

Figure 1. General location of the Eder acquisition.

Project boundaries, illustrated in Figure 2, were furnished by WDFW (J. Olson, pers. comm.) as "hard copy" maps. The boundaries were redrawn on Maptech ${ }^{\circledR}$ mapping software by Regional HEP Team staff. As a result, map boundaries depicted in Figure 2 may be up to \pm 300 feet in error except for the north boundary which is the international boundary with Canada. 


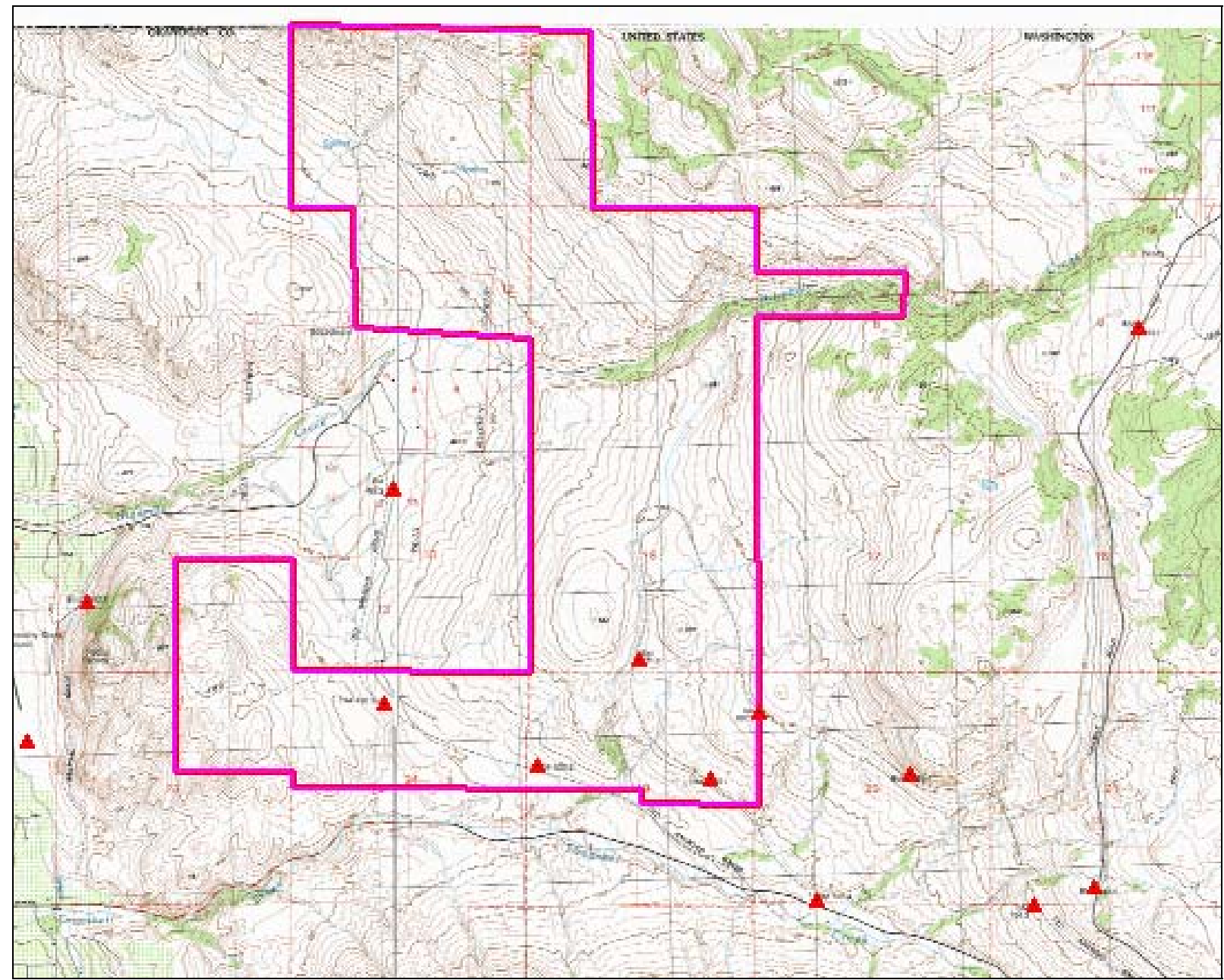

Figure 2. Eder acquisition project boundary.

\section{Topography}

Elevation ranges from approximately 1,500 feet to over 2,700 feet. Topography varies from flat pasture and rolling hills to steep stream channels and mountainous terrain dominated by rock outcrops.

\section{Cover Types}

Cover type maps were not available prior to initiation of the HEP analysis because there was insufficient time between the date of purchase and the HEP study for WDFW GIS staff to develop maps (P. Dahmer, pers. comm.). Therefore, RHT staff developed coarse cover type maps from aerial photographs and "ground-truthed" the maps while conducting HEP surveys.

Six primary cover types were delineated by Regional HEP Team staff i.e., shrubsteppe, grassland, rockland, riparian shrub, riparian forest, and conifer forest. Cover type acreages are shown in Table 1 and presented in Figure 3. 
Eder Acquisition 2007 HEP Report

Table 1. Eder property cover types, acres, and relative percent of area.

\begin{tabular}{|l|c|c|}
\hline \multicolumn{1}{|c|}{ Cover Type } & Acres & Percent of Area \\
\hline Shrubsteppe & 2,346 & 70 \\
\hline Grassland & 749 & 22 \\
\hline Rockland & 135 & 4 \\
\hline Riparian Shrub & 23 & $<1$ \\
\hline Riparian Forest & 66 & 2 \\
\hline Conifer Forest & 18 & $<1$ \\
\hline Total & $\mathbf{3 , 3 3 7}$ & $\approx \mathbf{1 0 0}$ \\
\hline
\end{tabular}


Eder Acquisition 2007 HEP Report

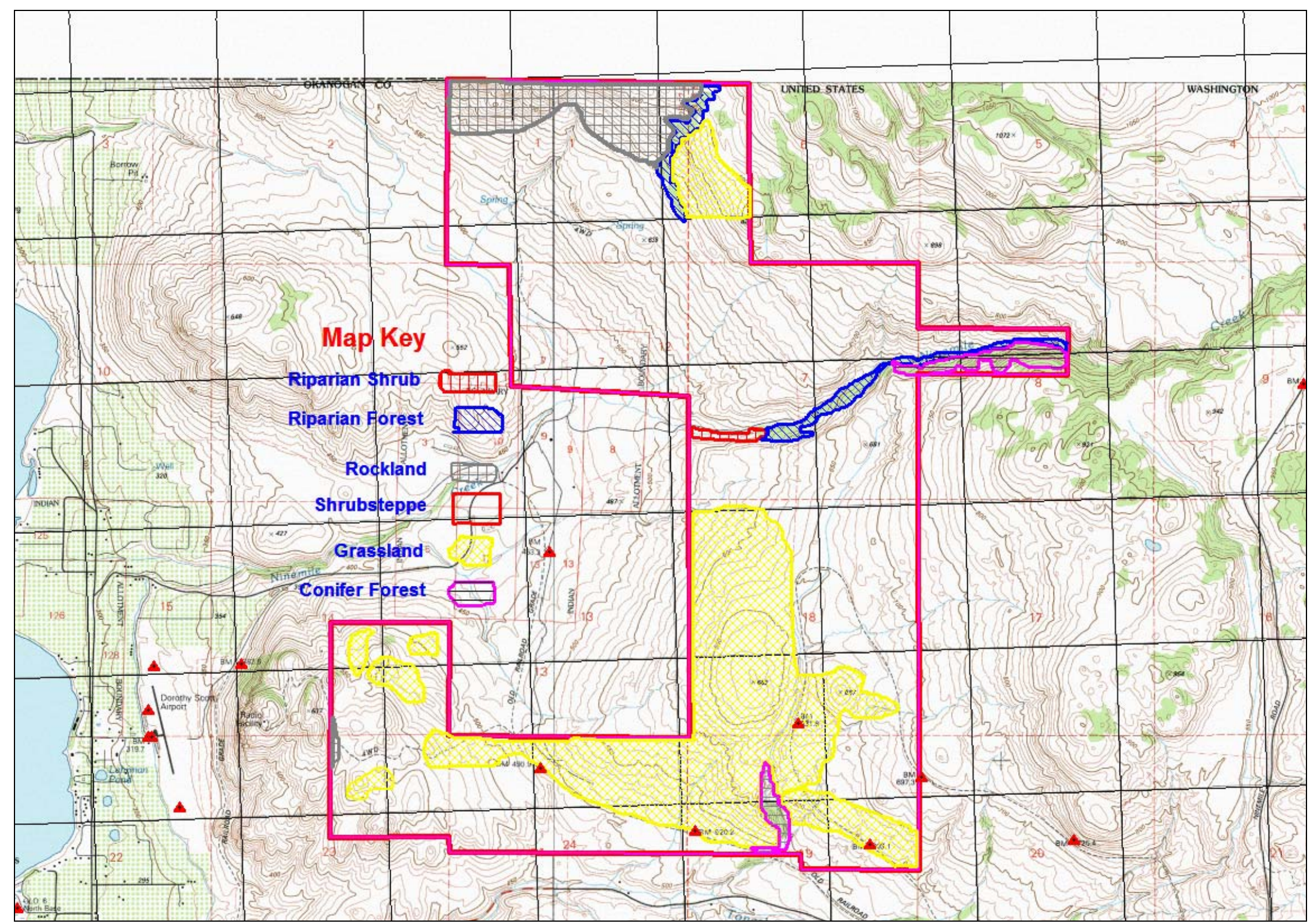

Figure 3. Eder property coarse filter cover type map.

Paul R Ashley

Page 5

$5 / 1 / 2008$ 


\section{Cover Type Descriptions}

Xeric uplands dominate the landscape with shrubsteppe comprising approximately $70 \%$ of the area. Grasslands cover 22\% while rockland, riparian shrub, riparian forest and conifer forest combined equal less than $8 \%$ of the project site. Rockland is found primarily along the north boundary with Canada. Riparian cover types occur either in conjunction with spring sites, or along Nine Mile Creek (Figure 3).

\section{Shrubsteppe}

The shrubsteppe cover type is comprised of xeric uplands with $\geq 5 \%$ shrub cover and $\leq 5 \%$ tree canopy. Although not typically observed all on the same transect, shrub species included big sagebrush (Artemisia tridentata), three-tip sagebrush (A. tripartite), bitterbrush (Purshia tridentata), green rabbitbrush (Chrysothamnus viscidiflorus), and currant (Ribes spp.). Shrubs were either dispersed relatively uniformly or as mosaics where shrubs were "clumped", interspersed with small openings dominated by herbaceous vegetation.

The herbaceous layer was comprised of both native and introduced species e.g., bluebunch wheatgrass (Pseudoroegneria spicata) and cheatgrass (Bromus tectorum) respectively. An example of shrubsteppe habitat is shown in Figure 4.

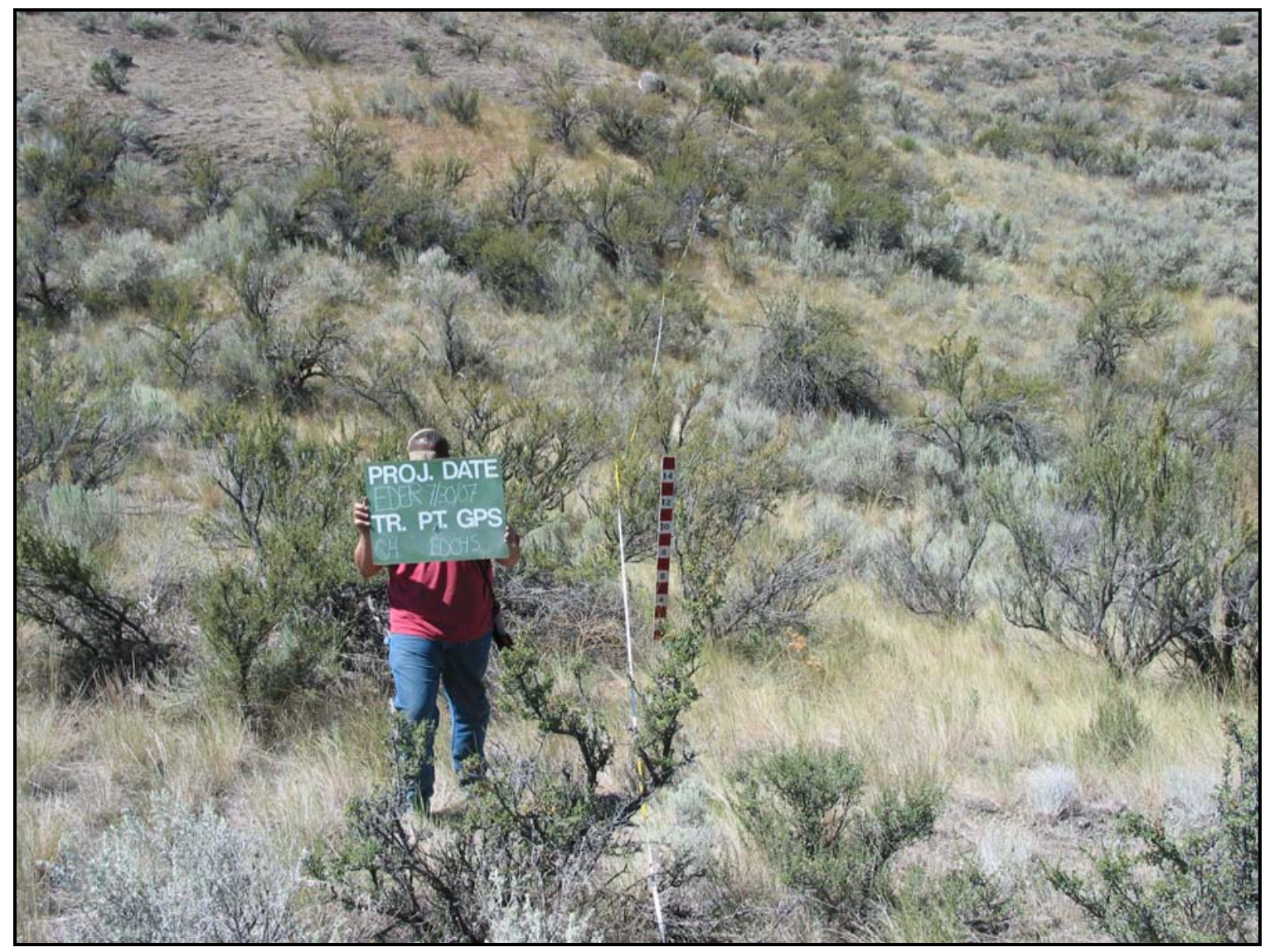

Figure 4. An example of the shrubsteppe cover type. 


\section{Grassland}

Grasslands generally occurred on upland sites and supported $<5 \%$ shrub and/or tree cover. Herbaceous cover was dominated by native and introduced grass species (few forbs were present most likely due to a combination of livestock grazing and the late timing of the surveys i.e., most forbs are desiccated by late July). Common native grass species observed were bluebunch wheatgrass, Sandberg bluegrass (Poa sandbergii), needle-and-thread (Stipa comata), and sand dropseed (Sporobolus cryptandrus).

Non-native herbaceous species included introduced pasture grasses, cheatgrass, and mustard (Sisymbrium spp.) to name a few. Transect results indicated that percent cover of exotic herbaceous species ranged from $<5 \%$ to more than $80 \%$. The grassland cover type is illustrated in Figure 5.

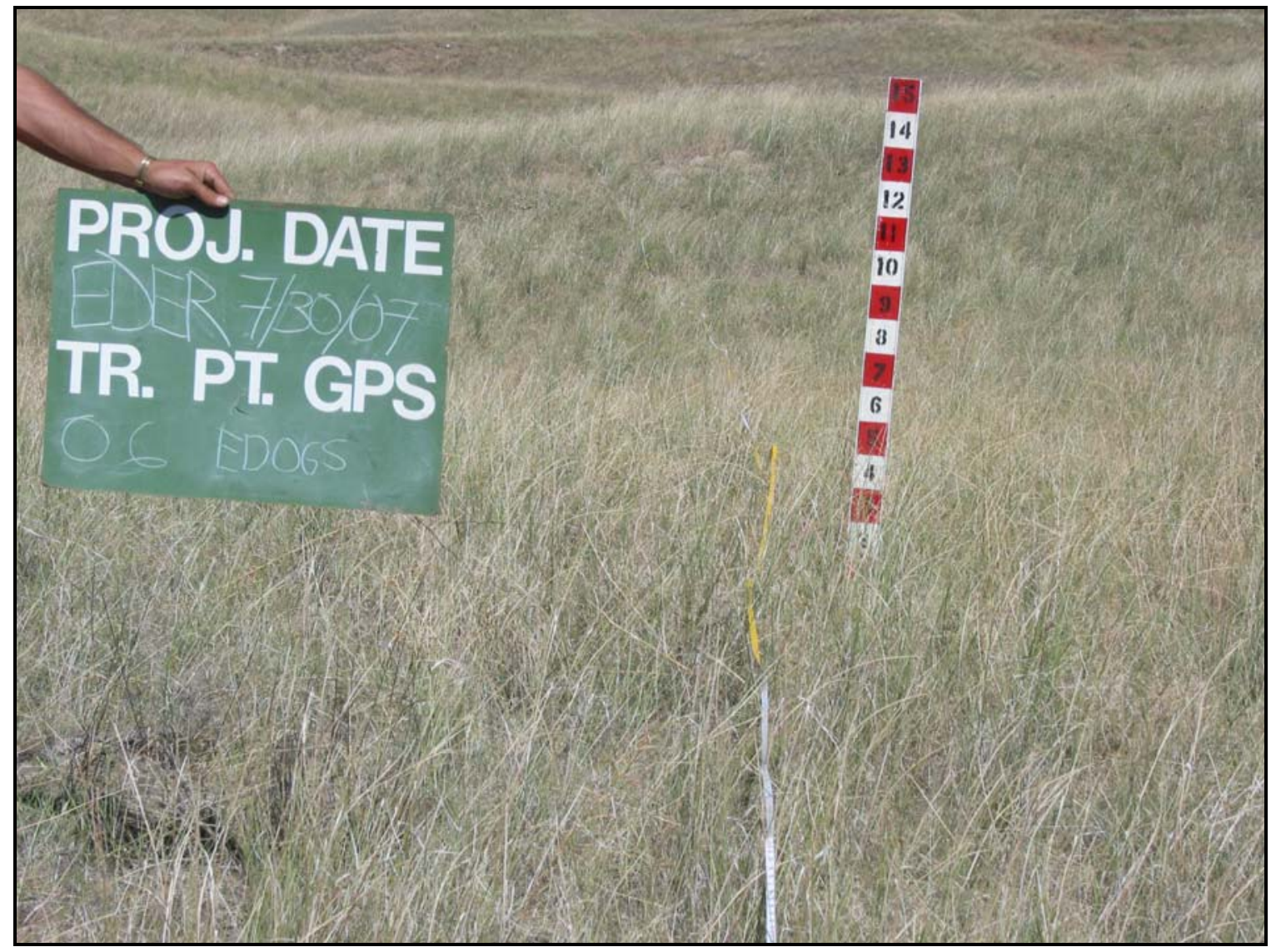

Figure 5. An example of the grassland cover type.

\section{Rockland}

The rockland cover type is characterized by boulders and cliffs interspersed with shrubs and/or trees. A herbaceous layer occurs where soils are present. Shrub cover is dominated by big sagebrush while ponderosa pine (Pinus ponderosa) trees are scattered throughout the cover type (Figure 6). 


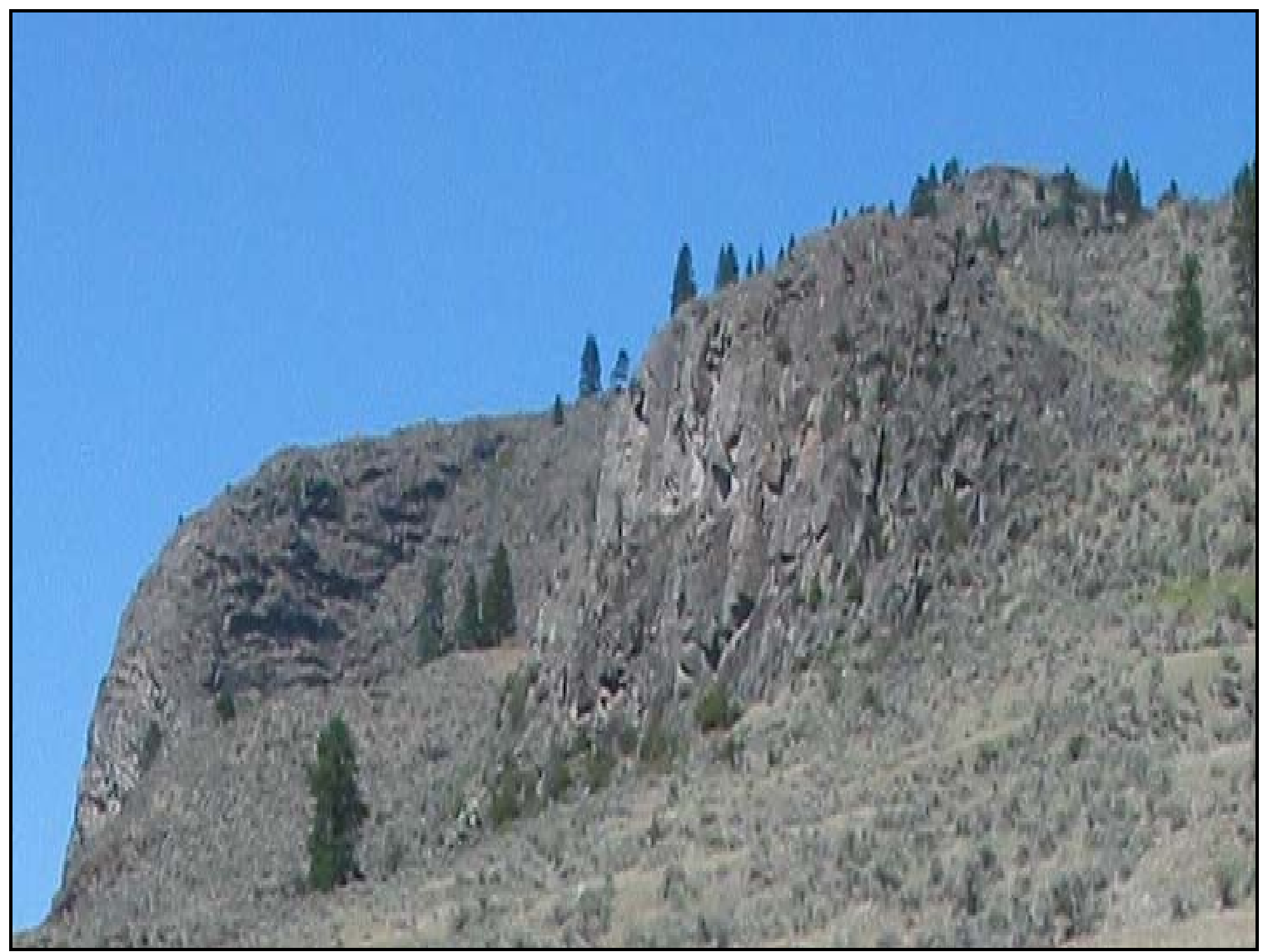

Figure 6. An example of the rockland cover type.

\section{Riparian Shrub}

Both hydrophytic and upland shrub species were present within the complex riparian shrub cover type. Shrub species varied by specific location, but all survey sites included rose (Rosa spp.) and snowberry (Symphoricarpus albus). Other shrubs ${ }^{1}$ observed were hawthorn (Crataegus douglassi), black cottonwood (Populus trichocarpa), mock orange (Philadelphus lewisii), chokecherry (Prunus virginiana), and clematis (Clematis ligusticifolia). In contrast, introduced Russian olive (Elaeagnus angustifolia) shrubs completely dominated one transect located in a mesic pasture (Transect 44). An example of the riparian shrub cover type is shown in Figure 7.

\footnotetext{
${ }^{1}$ All woody stemmed plants less than 16 feet in height were considered to function as shrubs regardless of species.
} 


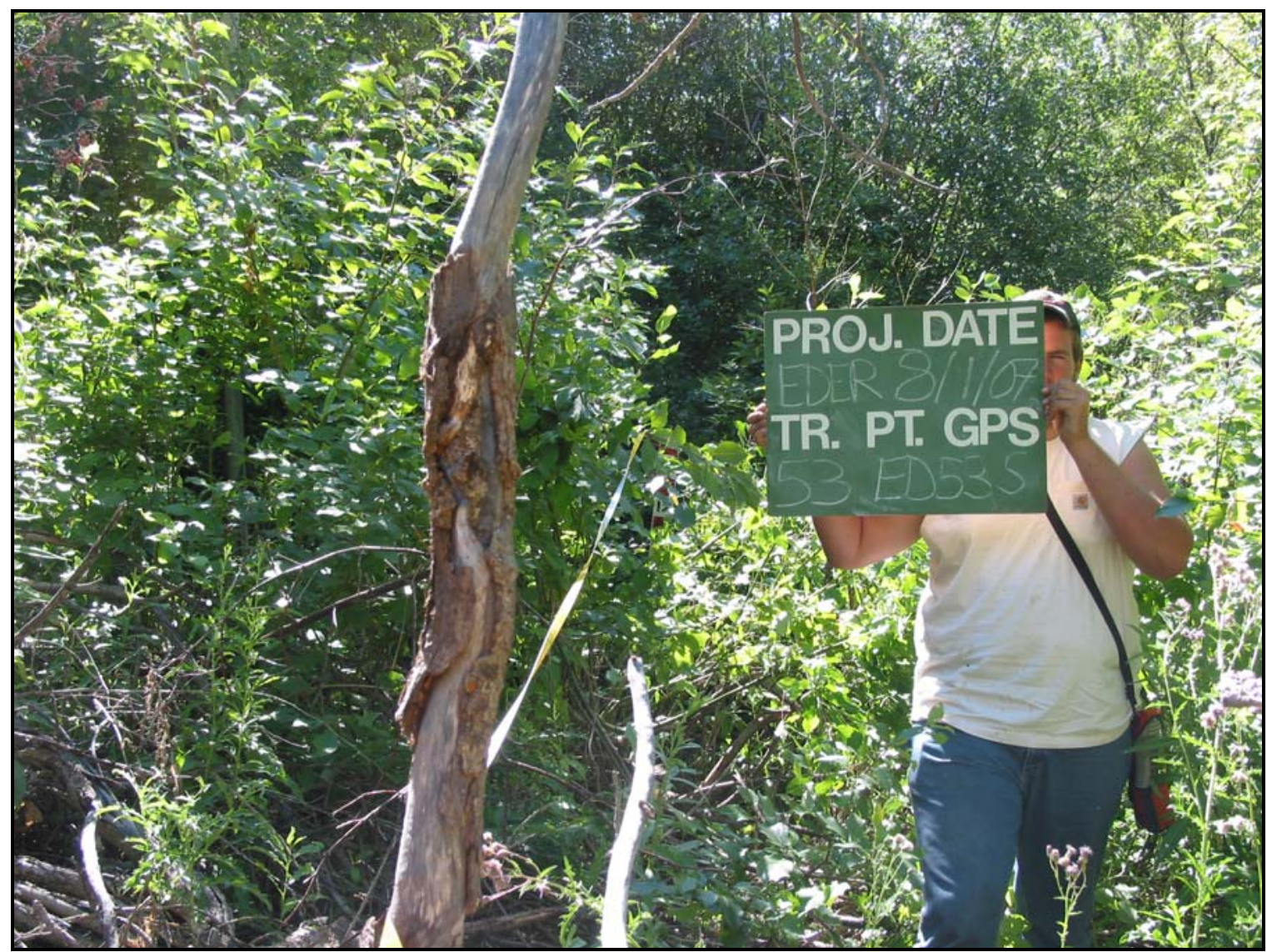

Figure 7. An example of riparian shrub understory.

\section{Riparian Forest}

Both hydrophytic and upland tree and shrub species were detected in the riparian forest cover type. Tree species included water birch (Betula occidentalis), aspen (Populus tremuloides), willow (Salix spp.), ponderosa pine, Douglas fir (Pseudotsuga menziesii), maple (Acer glabrum), black cottonwood, and alder (Alnus spp.). Tree species varied by transect with tree canopy cover ranging from $10 \%$ to $70 \%$.

Riparian forest shrub understory was similar to that described for the riparian shrub cover type. Understory shrub species included dogwood (Cornus stolonifera), aspen, rose, snowberry, maple, water birch, willow, mock orange, clematis, and serviceberry (Amelanchier alnifolia). The riparian forest cover type is illustrated in Figure 8. 


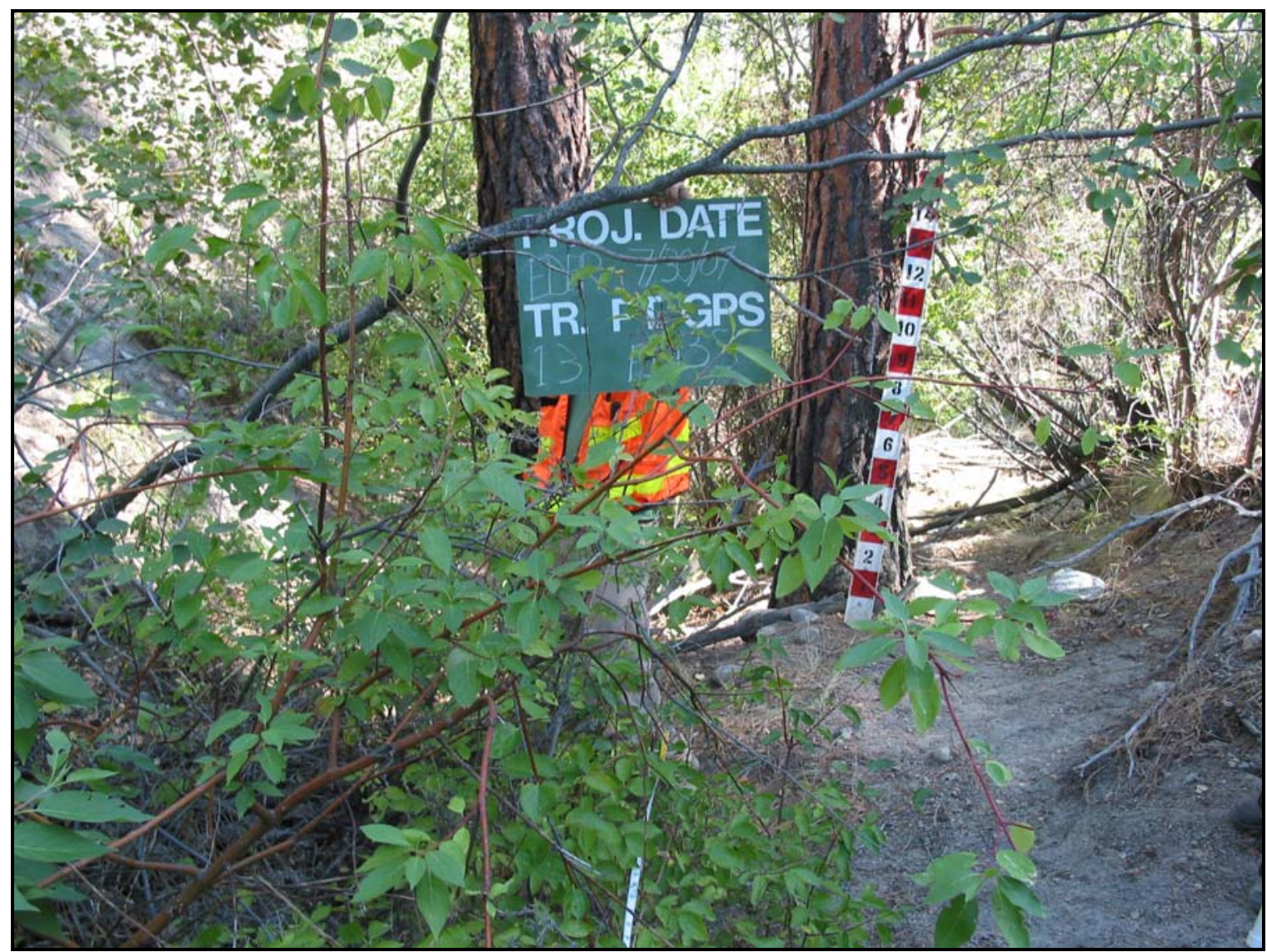

Figure 8. Riparian forest cover type photo.

\section{Conifer Forest}

The conifer forest cover type is dominated by ponderosa pine followed by Douglas fir trees. Shrubs may include sagebrush, bitterbrush, rose, snowberry, maple, mock orange, clematis, and serviceberry. This cover type was included with riparian forest in the HEP assessment because the same HEP evaluation species was applied to both cover types. No photograph is available for this cover type.

\section{Methods}

\section{Habitat Evaluation Procedures}

A habitat evaluation procedures analysis was conducted on the Eder acquisition to document baseline habitat conditions and to determine how many protection habitat units to credit BPA for providing funds to acquire the project site as partial mitigation for habitat losses associated with construction of Grand Coulee and Chief Joseph Dams. HEP, developed by the U.S. Fish and Wildlife Service (USFWS), is used to quantify the impacts of development, protection, and restoration projects/measures on terrestrial and aquatic habitats by assessing changes, both negative and positive, in habitat quality and quantity (USFWS 1980), (USFWS 1980a). 
HEP is a habitat based approach to impact assessment that documents change through use of a habitat suitability index (HSI). The HSI value is derived from an evaluation of the ability of key habitat components to provide the life requisites of selected wildlife and fish species.

The HSI value is an index to habitat carrying capacity for a specific species or guild of species based on a performance measure (e.g. number of deer per square mile) described in HEP species models. The index ranges from 0.0 to 1.0. A HSI of 0.3 indicates that habitat quality/carrying capacity is marginal while a HSI of 0.7 suggests that habitat quality/carrying capacity is relatively good for a particular species (Table 2 ).

Table 2. Habitat suitability index verbal equivalency table.

\begin{tabular}{|cc|}
\hline Habitat Suitability Index & Verbal Equivalent \\
\hline $0.0<0.2$ & Poor \\
$0.2<0.4$ & Marginal \\
$0.4<0.6$ & Fair \\
$0.6<0.9$ & Good \\
$0.9<1.0$ & Optimum \\
\hline
\end{tabular}

Each increment of change is identical. For example, a change in HSI from 0.1 to 0.2 represents the same magnitude of change as a change from 0.2 to 0.3 , and so forth. Habitat variables, suggested mensuration techniques, and mathematical aggregations of assessment results are included in HEP evaluation species models.

Habitat units are determined by multiplying the habitat suitability index by the number of acres of habitat (cover type) protected. For example, if the HSI output for a mule deer HEP model is 0.5 and the number of acres of shrubsteppe habitat protected is 100 , then the number of HUs are 50 (0.5 HSI x 100 acres $=50$ HUs $)$.

\section{HEP Model Selection}

HEP model selection was based on habitat types and species models identified in the Grand Coulee Dam (Howerton et al. 1986) and Chief Joseph Dam (Berger and Kuehn 1992) Loss Assessments. At Grand Coulee Dam, Howerton et al. (1986) did not clearly assign HEP species models to specific cover types making it difficult to develop a concise species/cover type matrix $^{2}$. In addition, contrary to HEP protocols two "cover type" HEP models ${ }^{3}$ (riparian shrub and riparian forest) were also included in the loss assessment.

Specific HEP models were not included in the Grand Coulee Dam Loss Assessment (Howerton et al. 1986) and were unavailable for the Eder HEP assessment. Therefore, models from other sources were used to evaluate the Eder wildlife mitigation site. In contrast, Berger and Kuehn (1992) included the bobcat HSI model in the Chief Joseph Dam Loss Assessment, which was used to evaluate the rockland cover type on the Eder parcel.

\footnotetext{
${ }^{2}$ The Coulee Dam species/cover type matrix is a draft document and subject to debate. It is, however, based on the best available data.

${ }^{3}$ By definition, cover types cannot be HEP models because HEP models must include a wildlife species.
} 
Consistent with other WDFW mitigation projects, HEP models selected by the Regional HEP Team to assess baseline habitat conditions included mule deer (Odocoileus hemionus) (Ashley and Berger 1996), western meadowlark (Sturnella neglecta) (Schroeder and Sousa 1982), sharptailed grouse, (Tympanuchus phasianellus) (Ashley 2003), Bobcat (Lynx rufus) (Bodurtha 1991) and Downy Woodpecker (Picoides pubescens) (Schroeder 1982). Abbreviated HEP models are included in Appendix A.

The 2007 Eder HEP evaluation cover type/species matrix shown in Table 3 is based primarily on information from the Grand Coulee Dam loss assessment (Howerton et al. 1986). The matrix also includes the rockland cover type and bobcat HSI model identified in the Chief Joseph Dam loss assessment (Berger and Kuehn 1992). As a result, bobcat habitat units were credited against Chief Joseph Dam while all other HU gains were credited against losses at Grand Coulee Dam.

Table 3. Eder project 2007 HEP loss assessment matrix.

\begin{tabular}{|c|c|c|c|c|c|c|c|}
\hline \multicolumn{8}{|c|}{ Eder 2007 HEP MODEL/COVER TYPE MATRIX } \\
\hline \multirow[b]{2}{*}{ HEP MODEL } & \multicolumn{7}{|c|}{ COVER TYPES } \\
\hline & Shrubsteppe & Grassland & Rockland & $\begin{array}{l}\text { Riparian } \\
\text { Shrub }\end{array}$ & $\begin{array}{c}\text { Riparian - } \\
\text { Conifer } \\
\text { Forest }\end{array}$ & $\begin{array}{l}\text { Coulee } \\
\text { Dam }\end{array}$ & $\begin{array}{c}\text { Chief } \\
\text { Joseph } \\
\text { Dam }\end{array}$ \\
\hline Mule deer & $\mathbf{x}$ & $\mathbf{x}$ & & & & $\mathbf{x}$ & \\
\hline Western meadowlark & $\mathbf{x}$ & $\mathbf{x}$ & & & & $\mathbf{x}$ & \\
\hline Sharp-tailed grouse & $\mathbf{x}$ & $\mathbf{x}$ & & $\mathbf{x}$ & & $\mathbf{x}$ & \\
\hline Bobcat & & & $\mathbf{x}$ & & & & $\mathbf{x}$ \\
\hline Downy Woodpecker & & & & & $\mathbf{x}$ & $\mathbf{x}$ & \\
\hline TOTAL & 3 & 3 & 1 & 1 & 1 & 4 & 2 \\
\hline
\end{tabular}

\section{HEP Species Model Selection Rationale}

Species selection rationale described in the Grand Coulee Dam Loss Assessment (Howerton et al. 1986) and from the Chief Joseph loss assessment (Berger and Kuehn 1992) is summarized in Table 4.

Table 4. HEP model species selection rationale table.

\begin{tabular}{|l|l|}
\hline \multicolumn{1}{|c|}{ HEP Model } & \multicolumn{1}{c|}{ Rationale } \\
\hline Mule deer & $\begin{array}{l}\text { This species represents wildlife dependent upon shrubsteppe and river } \\
\text { breaks. }\end{array}$ \\
\hline Western meadowlark & $\begin{array}{l}\text { Represents wildlife species dependent upon grassland and/or } \\
\text { shrubsteppe habitats. }\end{array}$ \\
\hline Sharp-tailed grouse & $\begin{array}{l}\text { Represents wildlife species dependent upon grasslands/shrubsteppe } \\
\text { habitat (includes riparian draws and limited agriculture). }\end{array}$ \\
\hline Bobcat & $\begin{array}{l}\text { Represents wildlife species dependent upon rocky areas and adjacent } \\
\text { grassland/shrubsteppe habitat. } \\
\text { The species represents wildlife dependent upon riparian forest habitats } \\
\text { and snags. }\end{array}$ \\
\hline
\end{tabular}


Eder Acquisition 2007 HEP Report

\section{Sampling Design and Measurement Protocols}

\section{Meta Data}

Level one meta data follows that suggested by Gotelli and Ellison (2004). Field surveys were conducted by the Columbia Basin Fish and Wildlife Authority Regional HEP Team with assistance from WDFW Wildlife Area staff Jim Olson and Bryan Dupont. Regional HEP Team members included Paul Ashley (RHT Coordinator), Mike Cantonese (Team Leader), Anthony Muse, Paul Walker, and Tiffany Baker (contact Paul Ashley@ lonepinebutte@,comcast.net, or through CBFWA at: [503] 229-0191).

Funding for the HEP analyses was provided by the Bonneville Power Administration with RHT administrative support provided by CBFWA. Specific measurement techniques and protocols are described in detail in Appendix B. Measurements were recorded in standard U.S. units except for the Robel pole (Robel et al. 1975), which was recorded in metric units.

\section{Transect Methods}

In most cases, the Regional HEP team used measurement techniques and protocols described in HEP models to evaluate habitat variables; however, ocular estimations were used when direct measurements could not be taken. Measured techniques were occasionally modified to meet unique habitat and/or physiographic conditions. Metrics generally followed those described by Hays et al. (1981) and/or Avery (1994).

Stratified (by cover type), random transects were established and documented using global positioning system (GPS) coordinates and, in many cases, rebar stakes. Ashley (2006) described the methods and protocols used by Regional HEP Team staff to collect HEP model variable data and additional floristic information (Appendix B). Field data was summarized and applied to HEP model variables to determine habitat suitability indices and habitat units for each HEP species model. Field data collection and processing procedures are illustrated in Figure 9 and summarized as follows.

HEP model variable field data was entered onto Allegro CE® data logger spreadsheets (1), or recorded on paper data sheets (2). The raw field data (3) was downloaded from the data loggers or manually entered from paper data sheets onto computers (transect photos were also downloaded and stored on field computers). The raw data and photos were compiled for each transect into three basic products/files (4) that are provided to project managers as report appendices and/or separate CD files.

Product files included raw field data downloaded from the data loggers (5), data summary spreadsheets (6) which are the results of compiling/processing the raw data, and transect photo files (7). Summarized/processed data from each transect was applied to appropriate HEP model variables to determine suitability index (SI) ratings that were combined on habitat suitability index (HSI) spreadsheets (8) to determine the HSI for a particular HEP species model/cover type. The habitat suitability index was then multiplied by the number of cover type acres to determine the number of habitat units (9). 


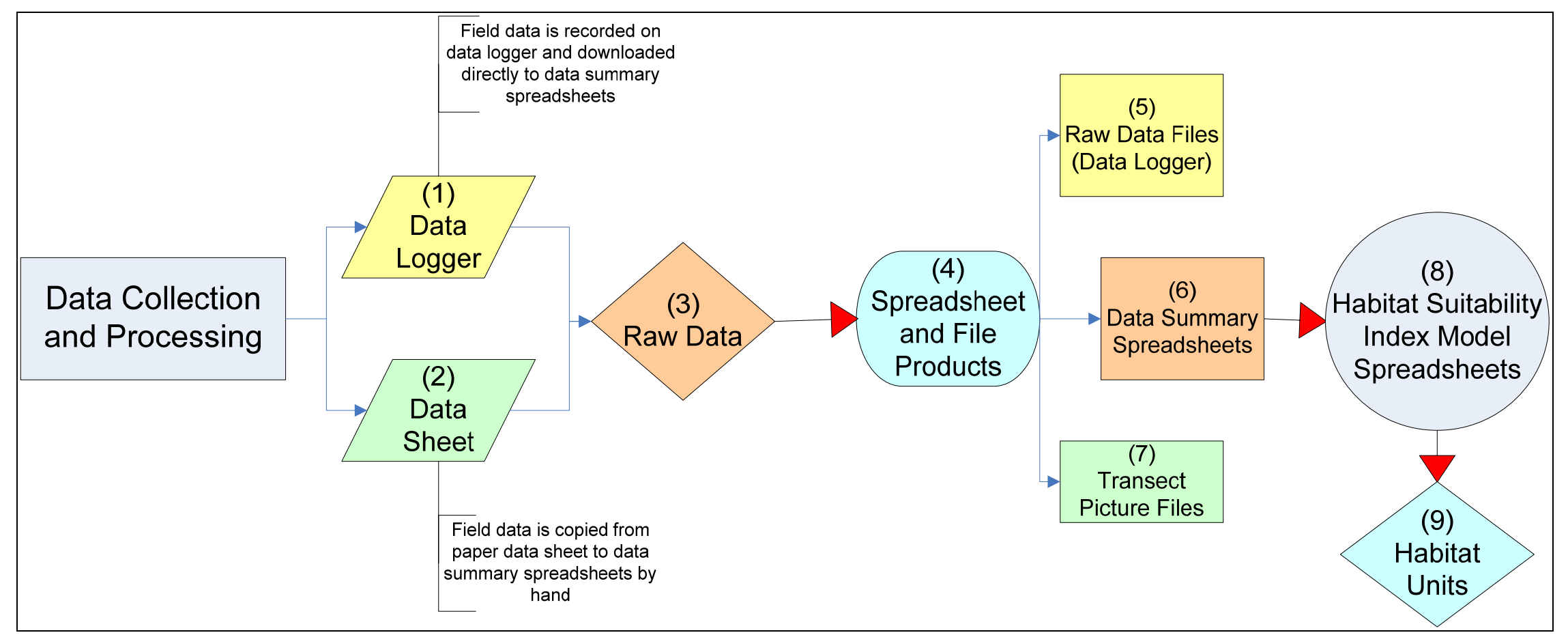

Figure 9. HEP data collection and processing flow chart. 
Eder Acquisition 2007 HEP Report

\section{Transect Locations}

Transect initial points (IPs) were established based on stratified random sampling protocols with cover types defining the strata. The number of samples initially allocated per cover type strata were determined based on a proportional allocation strategy (Husch et al. 2003). Specific IP locations were identified by overlaying a $100 \mathrm{~m} \times 100 \mathrm{~m}$ grid over cover types and selecting random numbers to identify "XY" point coordinates ( $\mathrm{P}$. Ashley, pers. comm.). Random IP locations are shown in Figure 10.

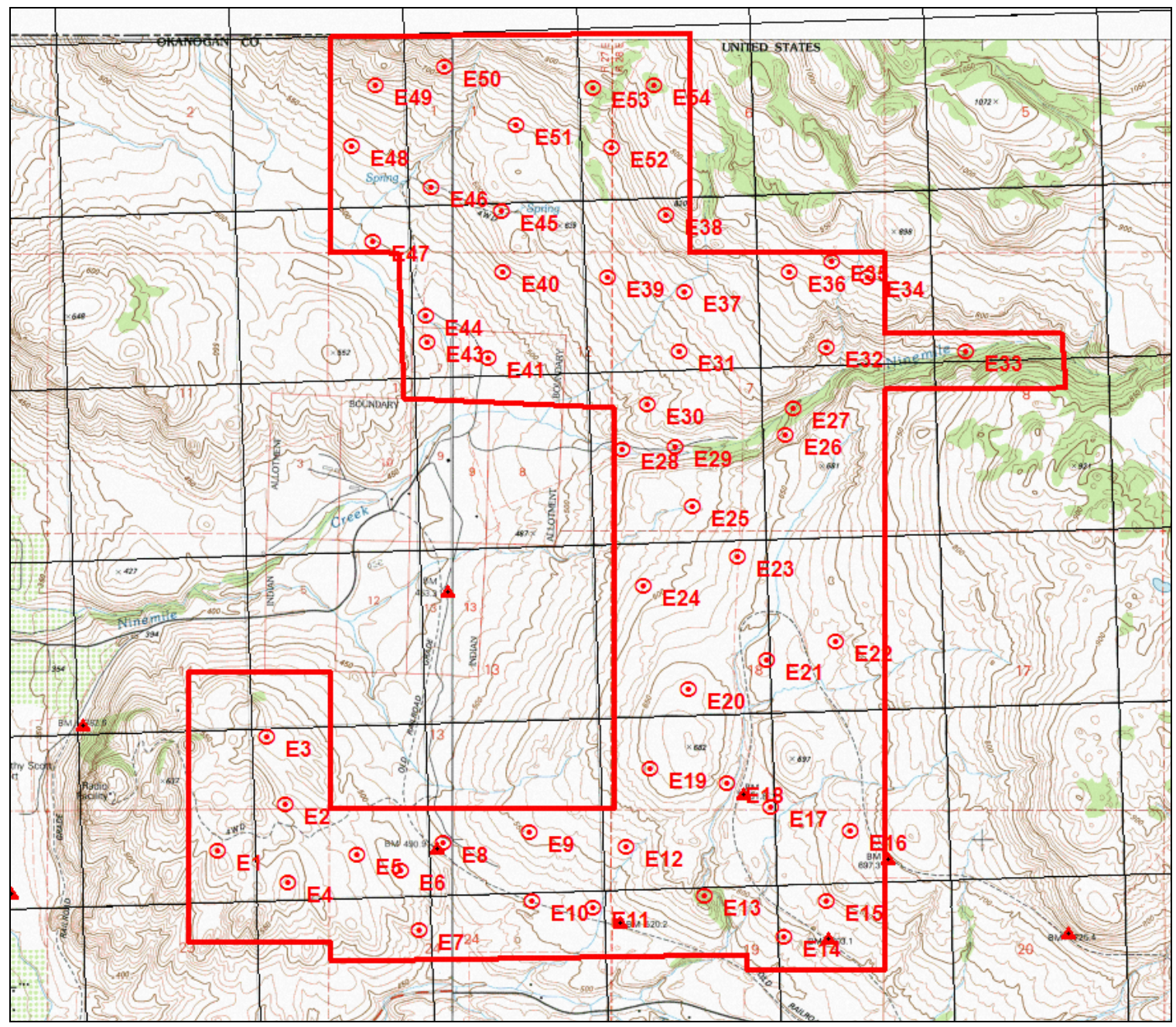

Figure 10. Eder project transect initial points.

The proportional allocation strategy was modified in the field as needed to compensate for the relative homogeneity of a particular cover type, to account for unanticipated access issues and/or physiographic restrictions, and/or to meet temporal considerations. In addition, initial points were moved when they did not fall within the cover type(s) of interest, or were in inaccessible areas such as the middle of a pond or cliff area (additional transect information is located in Appendix B). 
Transect UTM coordinates (NAD 27) for start, turn, and end points were recorded in the field on a Garmin IIIA ${ }^{\circledR}$ GPS unit. Surveyed transect start points are illustrated in Figure 11 (with UTM grid lines) and in Appendix C (aerial photographs). IP/transect UTM coordinates, transect magnetic azimuths, and transect lengths are summarized in Table 5.

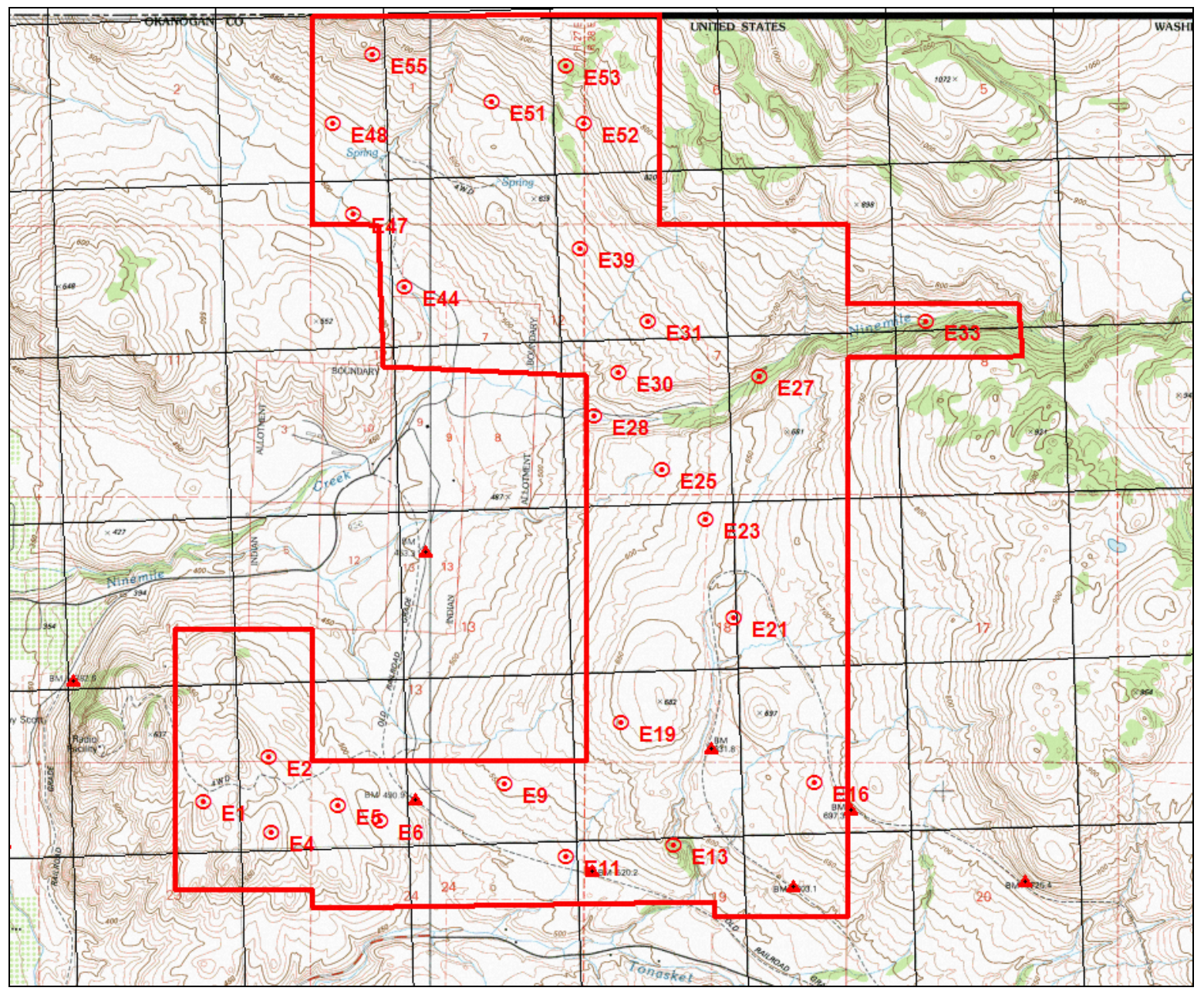

Figure 11. Eder property 2007 HEP transect start points. 
Eder Acquisition 2007 HEP Report

Table 5. Eder HEP transect UTM coordinates, magnetic azimuths, and transect lengths.

\begin{tabular}{|c|c|c|c|c|c|c|}
\hline \multirow{2}{*}{ Transect } & \multirow{2}{*}{ Point } & \multicolumn{2}{|l|}{ GPS } & \multirow{2}{*}{$\begin{array}{l}\text { Magnetic } \\
\text { Azimuth } \\
\text { (Degrees) }\end{array}$} & \multirow{2}{*}{$\begin{array}{l}\text { Length } \\
\text { (Feet) }\end{array}$} & \multirow{2}{*}{ Total Length } \\
\hline & & E & $\mathrm{N}$ & & & \\
\hline \multirow[t]{2}{*}{1} & start & $11 \cup 0324708$ & 5425250 & 240 & 300 & 300 \\
\hline & end & $11 \cup 0324710$ & 5425298 & & & \\
\hline \multirow[t]{2}{*}{2} & start & $11 \cup 0325204$ & 5425568 & 260 & 300 & 300 \\
\hline & end & $11 \cup 0325124$ & 5425602 & & & \\
\hline \multirow[t]{2}{*}{4} & start & $11 \cup 0325020$ & 5425181 & 256 & 300 & 300 \\
\hline & end & $11 \cup 0324925$ & 5425195 & & & \\
\hline \multirow[t]{2}{*}{5} & start & $11 \cup 0325611$ & 5425236 & 132 & 300 & 300 \\
\hline & end & $11 \cup 0325564$ & 5425317 & & & \\
\hline \multirow[t]{2}{*}{6} & start & $11 \cup 0325848$ & 5425148 & 024 & 300 & 300 \\
\hline & end & $11 \cup 0325907$ & 5425207 & & & \\
\hline \multirow[t]{2}{*}{9} & start & $11 \cup 0326598$ & 5425361 & 178 & 300 & 300 \\
\hline & end & $11 \cup 0327402$ & 5425782 & & & \\
\hline \multirow[t]{2}{*}{11} & start & $11 \cup 0326945$ & 5424905 & 040 & 300 & 300 \\
\hline & end & $11 \cup 0327022$ & 5424905 & & & \\
\hline \multirow[t]{2}{*}{13} & start & $11 \cup 0327675$ & 5424933 & Green line & 300 & 300 \\
\hline & end & $11 \cup 0327705$ & 5424831 & & & \\
\hline \multirow[t]{2}{*}{16} & start & $11 \cup 0328441$ & 5425308 & 070 & 300 & 300 \\
\hline & end & $11 \cup 0328528$ & 5425310 & & & \\
\hline \multirow[t]{2}{*}{19} & start & $11 \cup 0327345$ & 5425700 & 015 & 300 & 300 \\
\hline & end & $11 \cup 0327402$ & 5425782 & & & \\
\hline \multirow[t]{2}{*}{21} & start & $11 \cup 0328004$ & 5426314 & 342 & 300 & 300 \\
\hline & end & $11 \cup 0328018$ & 5426425 & & & \\
\hline \multirow[t]{2}{*}{23} & start & $11 \cup 0327840$ & 5426901 & 348 & 300 & 300 \\
\hline & end & $11 \cup 0327856$ & 5427002 & & & \\
\hline \multirow[t]{2}{*}{25} & start & $11 \cup 0327607$ & 5427204 & 126 & 300 & 300 \\
\hline & end & Coordinates unavailable & & & & \\
\hline \multirow[t]{2}{*}{27} & start & $11 \cup 0328232$ & 5427816 & Green line & 300 & 300 \\
\hline & end & $11 \cup 0328178$ & 5427733 & & & \\
\hline \multirow[t]{2}{*}{28} & start & $11 \cup 0327215$ & 5427525 & Green line & 300 & 600 \\
\hline & end & $11 \cup 0327294$ & 5427531 & & & \\
\hline \multirow[t]{2}{*}{30} & start & $11 \cup 0327350$ & 5427801 & 138 & 300 & 300 \\
\hline & end & $11 \cup 0327399$ & 5427707 & & & \\
\hline \multirow[t]{2}{*}{31} & start & $11 \cup 0327549$ & 5428107 & 276 & 300 & 300 \\
\hline & end & $11 \cup 0327479$ & 5428147 & & & \\
\hline \multirow[t]{2}{*}{33} & start & 11 U 0329201 & 5428059 & Green line & 300 & 300 \\
\hline & end & $11 \cup 0329095$ & 5428056 & & & \\
\hline 39 & start & $11 \cup 0327152$ & 5428551 & 346 & 300 & 300 \\
\hline & end & $11 \cup 0327160$ & 5428642 & & & \\
\hline 44 & start & $11 \cup 0326095$ & 5428358 & 158 & 190 & 300 \\
\hline & turn & $11 \cup 0326113$ & 5428302 & 100 & 110 & \\
\hline & end & $11 \cup 0326135$ & 5428291 & & & \\
\hline
\end{tabular}


Eder Acquisition 2007 HEP Report

\begin{tabular}{|c|c|c|c|c|c|c|}
\hline \multirow{2}{*}{ Transect } & \multirow{2}{*}{ Point } & \multicolumn{2}{|l|}{ GPS } & \multirow{2}{*}{$\begin{array}{c}\text { Magnetic } \\
\text { Azimuth } \\
\text { (Degrees) }\end{array}$} & \multirow{2}{*}{$\begin{array}{c}\text { Length } \\
\text { (Feet) }\end{array}$} & \multirow{2}{*}{ Total Length } \\
\hline & & E & $\mathbf{N}$ & & & \\
\hline 48 & start & $11 \cup 0325694$ & 5429350 & 307 & 300 & 300 \\
\hline 51 & start & $11 \cup 0326650$ & 5429453 & ocular & & \\
\hline \multirow[t]{2}{*}{53} & start & $11 \cup 0327101$ & 5429644 & 120 & 300 & 300 \\
\hline & end & $11 \cup 0327156$ & 5429586 & & & \\
\hline 55 & start & $11 \cup 0325948$ & 5429750 & ocular & & \\
\hline \multirow[t]{2}{*}{69} & start & $11 \cup 0326362$ & 5427943 & 051 & 300 & 300 \\
\hline & end & $11 \cup 0326478$ & 5427957 & & & \\
\hline
\end{tabular}

\section{Transect Photo Documentation}

Transects were photographed with a Canon G1® 3.3 mega pixal digital camera (with and without magnification). Transect photographs are included in Appendix D.

\section{Photo Methods}

Photo points were established at the start point of each transect to document extant habitat conditions. Digital photographs were recorded from a height of three feet at the beginning of each transect facing the same direction as the transect azimuth. A transect reference board ${ }^{4}$ was placed at the 15 foot interval while a cover board, divided into 3 inch $\mathrm{x} 4$ inch $(8 \mathrm{~cm} \times 10 \mathrm{~cm})$ rectangles, was set at the 30 foot mark on each transect. Panoramic photographs were also recorded to document dense vegetation, linear/narrow cover types, etc. An example of a photo documentation point is illustrated in Figure 12.

\footnotetext{
${ }^{4}$ Showing transect number, project name, date, GPS reference number
} 


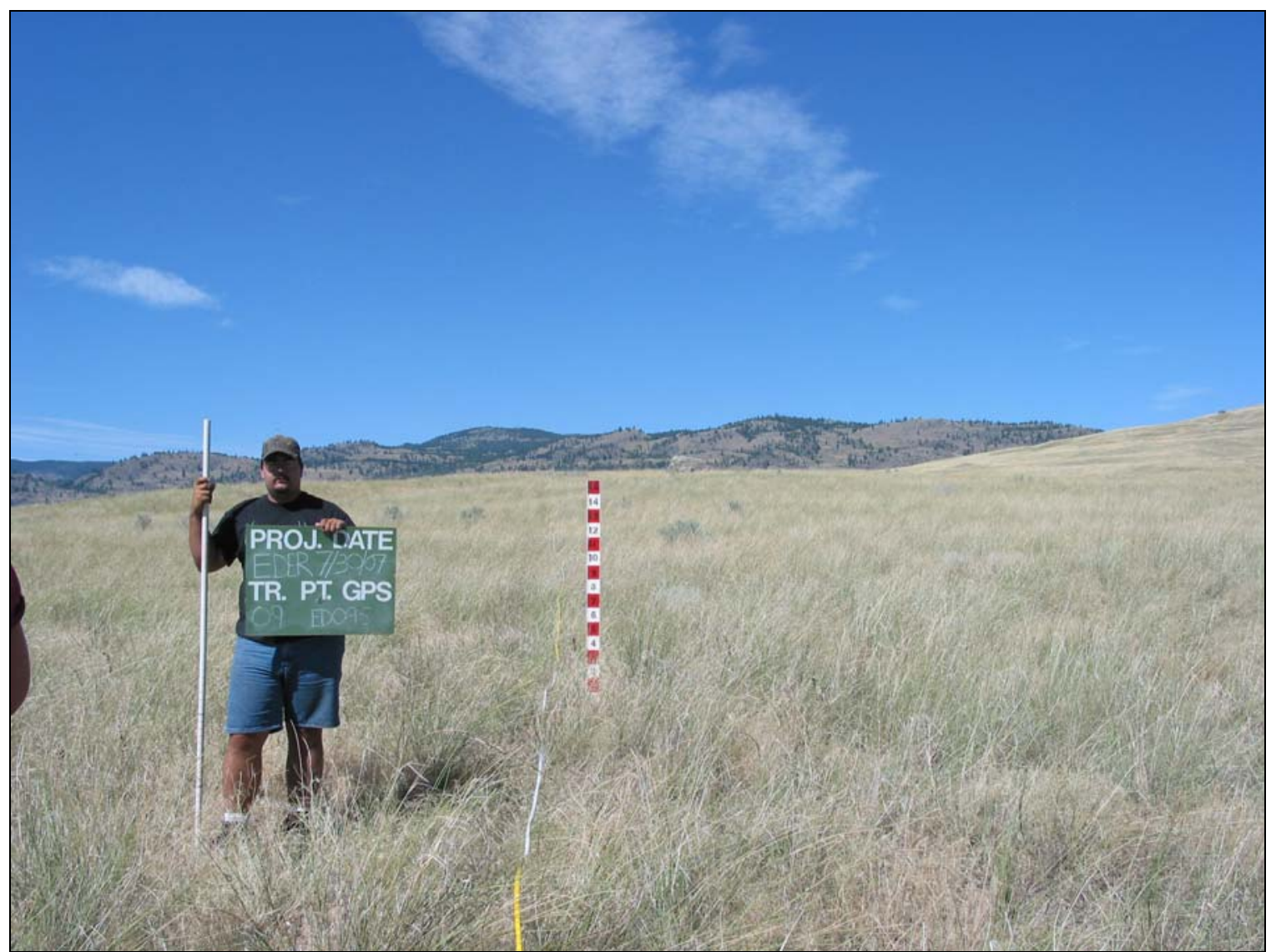

Figure 12. Photo point example.

\section{Results}

A Habitat Evaluation Procedures evaluation was conducted on the Eder property in late July 2007 to assess habitat quality and to determine the number of baseline/protection habitat units (HUs) to credit BPA as partial mitigation for habitat losses associated with Grand Coulee and Chief Joseph Dams. Baseline HEP surveys generated 3,857.64 habitat units or 1.16 HUs per acre. HEP survey results are summarized by cover types and species in Table 6. HEP species models and habitat suitability mathematical aggregations are included in Appendix A. 
Eder Acquisition 2007 HEP Report

Table 6. Eder acquisition 2007 HEP results summary.

\begin{tabular}{|c|c|c|c|c|c|c|c|c|c|c|}
\hline \multirow{2}{*}{ HEP Model } & \multirow{2}{*}{ Variable } & \multicolumn{4}{|c|}{ Shrubsteppe } & \multicolumn{4}{|c|}{ Grassland } & \multirow{2}{*}{ Total HUs } \\
\hline & & Mean SI & HSI & Acres & HUs & Mean SI & HSI & Acres & HUs & \\
\hline \multirow{5}{*}{ Western Meadowlark } & V1: \% C.C. Herb. Plants & 0.46 & 0.33 & 2,346 & 783.53 & 0.82 & 0.82 & 749 & 611.78 & $1,395.31$ \\
\hline & V2: \% Herb. C.C. Composed of Grass & 0.93 & & & & 0.93 & & & & \\
\hline & V3: Ave. Ht. of Herb. Canopy & 0.85 & & & & 0.88 & & & & \\
\hline & V4: Distance to Perch Sites & 1.00 & & & & 1.00 & & & & \\
\hline & V5: \% Shrub Canopy Cover & 0.56 & & & & 1.00 & & & & \\
\hline \multirow{10}{*}{ Sharp-tailed Grouse } & V1: Mean VOR - Landscape (all vegetation including residual) & 0.16 & 0.39 & 2,346 & 913.98 & 0.15 & 0.36 & 749 & 268.24 & $1,182.22$ \\
\hline & V2: Percent Slope & 0.77 & & & & 0.86 & & & & \\
\hline & V3: Percent Cover Grass & 0.80 & & & & 1.00 & & & & \\
\hline & V4: Percent Cover Forbs & 0.12 & & & & 0.18 & & & & \\
\hline & V5: Percent Cover Introduced Herbaceous Species & 0.56 & & & & 0.43 & & & & \\
\hline & V6: Percent Equivalent Optimum Area Providing Nest/Brood Cover & 1.00 & & & & 1.00 & & & & \\
\hline & V7: Distance Between Nesting/Brood Rearing and Winter Habitat & 1.00 & & & & 1.00 & & & & \\
\hline & V8: Percent Cover Deciduous shrubs and Trees & (see riparian shrub) & & & & & & & & \\
\hline & V9: Deciduous Shrub and Tree Composition/Wheat Availability & (see riparian shrub) & & & & & & & & \\
\hline & V10: Percent Equivalent Optimum Area Providing Winter Habitat & (see riparian shrub) & & & & & & & & \\
\hline \multirow{10}{*}{ Mule Deer } & $\begin{array}{l}\text { V1: Percent cover of preferred } \\
\text { shrubs }<1.5 \text { meters in height }\end{array}$ & 0.53 & 0.43 & 2,346 & $1,005.72$ & 0.01 & 0.17 & 749 & 130.04 & $1,135.77$ \\
\hline & V2: Percent cover of all shrubs $<1.5$ meters in height. & 0.53 & & & & 0.01 & & & & \\
\hline & V3: Mean shrub height. & 0.47 & & & & 0.11 & & & & \\
\hline & V4: Number of preferred shrub species. & 0.62 & & & & 0.06 & & & & \\
\hline & V5: Percent cover of palatable herbaceous species. & 0.93 & & & & 1.00 & & & & \\
\hline & $\begin{array}{l}\text { V6: Presence of suitable agricultural crops } \\
\text { within } 1.6 \text { kilometers ( } 1 \text { mile) of study area }\end{array}$ & 0.10 & & & & 0.10 & & & & \\
\hline & V7: Aspect & 0.60 & & & & 0.60 & & & & \\
\hline & V8: Road density & 1.00 & & & & 1.00 & & & & \\
\hline & V9: Topographic diversity & 1.00 & & & & 1.00 & & & & \\
\hline & $\begin{array}{l}\text { V10: Percent evergreen canopy } \\
>1.5 \text { meters in height }\end{array}$ & 0.00 & & & & 0.00 & & & & \\
\hline \multirow[t]{2}{*}{ Total } & & & & 2,346 & $2,703.23$ & & & 749 & $1,010.06$ & $3,713.29$ \\
\hline & & \multicolumn{4}{|c|}{ Rockland } & & & & & \\
\hline \multirow{4}{*}{ Bobcat } & V1: Percent cover herbaceous vegetation & 0.80 & 0.73 & 135 & 99.00 & & & & & 99.00 \\
\hline & V2: Shrub distribution & 0.70 & & & & & & & & \\
\hline & V3: Percent shrub cover & 0.30 & & & & & & & & \\
\hline & V4: Percent area comprised of rock outcrops, boulders, etc. & 1.00 & & & & & & & & \\
\hline \multirow[t]{2}{*}{ Total } & & & & 135.00 & 99.00 & & & & & 99.00 \\
\hline & & \multicolumn{4}{|c|}{ Riparian Shrub } & & & & & \\
\hline \multirow{3}{*}{ Sharp-tailed Grouse (winter) } & V8: Percent Cover Deciduous shrubs and Trees & 0.45 & 0.05 & 23 & 1.24 & & & & & 1.24 \\
\hline & V9: Deciduous Shrub and Tree Composition/Wheat Availability & 0.65 & & & & & & & & \\
\hline & V10: Percent Equivalent Optimum Area Providing Winter Habitat & 0.10 & & & & & & & & \\
\hline \multirow[t]{2}{*}{ Total } & & & & 23.00 & 1.24 & & & & & 1.24 \\
\hline & & \multicolumn{4}{|c|}{ Riparian/Conifer Forest } & & & & & \\
\hline \multirow[b]{2}{*}{ Downy Woodpecker } & V1: Basal Area & 0.53 & 0.53 & 84 & 44.10 & & & & & 44.10 \\
\hline & $\begin{array}{l}\text { V2: Number of snags }>15 \mathrm{~cm} \mathrm{dbh} / 0.4 \mathrm{ha} \text { ( }>6 \text { inches } \mathrm{dbh} / \\
1.0 \text { acre). }\end{array}$ & 0.75 & & & & & & & & \\
\hline Total & & & & 84 & 44.10 & & & & & 44.10 \\
\hline Project Total & & & & 3,337 & & & & & & $3,857.64$ \\
\hline
\end{tabular}

Paul R Ashley 
Eder Acquisition 2007 HEP Report

\section{Discussion}

\section{HSI Summary}

Comments are limited to HEP model species that received a habitat suitability index rating less than 0.50 . Western meadowlark, bobcat, and downy woodpecker habitat suitability indices were 0.82 (grassland cover type), 0.73 and 0.53 respectively and, therefore, will not be addressed in this section (Table 6).

In general, few forbs species were detected on transects which may have been due to a combination of livestock grazing and the late timing of the surveys. Similarly, visual obstruction readings (VOR) were lower than expected; likely a direct result of livestock impacts. Exotic herbaceous species including cultivated pasture grasses e.g., crested wheatgrass (Agropyron cristatum) and/or invader species such as cheatgrass and mustard were observed in most areas surveyed.

\section{Western Meadowlark}

Western meadowlark model output suggests that habitat quality within the shrubsteppe cover type was marginal (0.33 HSI) largely because of the amount of shrub cover present and the relatively low suitability index for variable 1, "percent cover of herbaceous species". When livestock grazing ceases and/or is reduced significantly, herbaceous cover should increase resulting in improved western meadowlark habitat suitability. In contrast, shrub cover will likely remain static, or increase only slightly.

\section{Sharp-tailed Grouse}

Sharp-tailed grouse habitat suitability was marginal in both grassland and shrubsteppe cover types (0.36 HSI and 0.39 HSI respectively). In both cover types, low VOR was the primary factor limiting nesting and brood rearing habitat quality suitability. Less than desirable floristic composition resulting from the relatively high occurrence of non-native invasive plant species and the lack of forbs also contributed towards reduced HSI ratings.

The low habitat suitability rating generated in the riparian shrub cover type $(<0.10 \mathrm{HSI})$, which provides winter food and escape cover, was due primarily to the limited extent of this cover type on project lands. Increasing the amount of the riparian shrub cover type would increase the model HSI and generate additional habitat units.

If adjacent off-site areas were considered in HEP evaluations, i.e. at the landscape level, the sharp-tailed grouse winter habitat suitability index would likely increase because winter habitat is present on adjacent lands. To date, project managers throughout the Columbia Basin have included only mitigation lands in HEP evaluations as a result of the well founded concern for lack of management control on adjacent privately owned lands. It could be argued, however, that adjacent public lands or lands held in "Trust" should be considered in HEP evaluations if Tribal, Federal, or State management mandates and/or statutes protect habitat quality. 


\section{Mule Deer}

Currently, mule deer habitat quality is "fair" (0.43 HSI) in the shrubsteppe cover type. Passive management that allows the percent cover of palatable shrubs to increase would improve winter foraging conditions for mule deer and HEP model HSI output. In contrast, mule deer habitat quality is rated "poor" $(0.17 \mathrm{HSI})$ in the grassland cover type due to the lack of palatable shrubs. If WDFW management objectives call for keeping grassland structure intact i.e., less than 5\% shrub cover, then the HSI will remain "static".

Note that "percent palatable shrub cover" (V1) is a key habitat variable that significantly influences the mule deer HEP model output. If shrubs are not present or limited, the model HSI will be low even if all other variables are optimum. This artificially constrains model habitat suitability if applied to cover types with limited shrub cover. Modifying the existing HEP model to include rating habitat variables at the landscape level, similar to what was done with the white-tailed deer model on the Spokane Indian Reservation (Ashley 2005), is a biologically reasonable option to address use of the model in cover types that lack palatable shrubs, but are still used by mule deer.

\section{Acknowledgements}

I gratefully acknowledge the hard work and effort provided by WDFW Scotch Creek Wildlife Area staff Jim Olson and Bryan Dupont and Regional HEP Team members Mikael Cantonese, Tiffany Baker, Tony Muse, and Paul Walker. 
Eder Acquisition 2007 HEP Report

\section{References}

Ashley, P. R., and M. Berger 1999. Habitat suitability model mule deer (winter). Olympia WA: Washington Department of Fish and Wildlife. Nespelem, WA: Colville Confederated Tribes.

Ashley, P. R. 2003. Sharp-tailed grouse HEP model. Olympia WA: Washington Department of Fish and Wildlife.

. 2005. White-tailed deer HEP model (draft). Portland, OR: Columbia Basin Fish and Wildlife Authority. Wellpinit, WA: Spokane Tribe of Indians.

. 2006. Habitat evaluation procedures standard measurement protocols and techniques (draft). Columbia Basin Fish and Wildlife Authority (CBFWA). Portland, OR.

Avery, T.E., H. E. Burkhart. 1994. Forest measurements. $4^{\text {th }}$ edition. New York, NY: John Wiley and Sons.

Berger, M. T. and D. Kuehn. 1992. Wildlife impact assessment Chief Joseph Dam Project. Project N0. 88-44. Bonneville Power Administration. Portland, OR.

Bodurtha, Tim. 1991. Unpublished habitat suitability index model: Bobcat. U. S. Fish and Wildlife Service.

BPA/WDFW. 1996. Memorandum of Agreement between the Washington Department of Fish and Wildlife and Bonneville Power Administration for the disbursal of wildlife mitigation funds and mitigation crediting. WDFW. Olympia, WA. BPA. Portland, OR.

Gotelli, N. J., A. M. Ellison. 2004. A primer of ecological statistics. Sinauer Associates, Inc. Sunderland, MA.

Hays, R. L., C. Summers, and W. Seitz. 1981. Estimating habitat variables. Western Energy and land Use Team. Fort Collins, CO: U.S. Fish and Wildlife Service.

Howerton, J., J. Creveling, and B. Renfrow. 1986. Wildlife protection, mitigation, and enhancement planning for Grand Coulee Dam. Olympia, WA: Washington Department of Fish and Wildlife.

Husch, B., T.W. Beers, and J.A. Kershaw, Jr. 2003. Forest mensuration- $4^{\text {th }}$ edition. Hoboken, NJ: Wiley and Sons, Inc.

Robel, R.J., J. N. Dayton, A.D. Hulbert. 1975. Relationship between visual obstruction measurements and weight of grassland vegetation. Journal of Range Management. 23: 295. 
Eder Acquisition 2007 HEP Report

Schroeder, R.L. 1982. Habitat suitability index models:

Downy woodpecker. U.S. Department of the Interior, Fish and Wildlife Service. FWS/OBS-82/10.38.

Schroeder, R.L., and P.J. Sousa. 1982. Habitat suitability index models:

Eastern meadowlark. U.S. Department of the Interior, Fish and Wildlife

Service. FWS/OBS-82/10.29.

USFWS. 1980. Habitat as a Basis for Environmental Assessment, Ecological Services Manual (ESM) 101. Division of Ecological Services, U. S. Fish and Wildlife Service, Washington, DC: Department of the Interior.

- 1980a. Habitat Evaluation Procedures (HEP), Ecological Services Manual (ESM) 102. Division of Ecological Services, U.S. Fish and Wildlife Service, Washington, DC: Department of the Interior. 


\section{Appendix A - Abbreviated HEP Models}

\section{Mule Deer}

V1: Percent palatable shrub cover

$<5 \mathrm{ft}$ in height

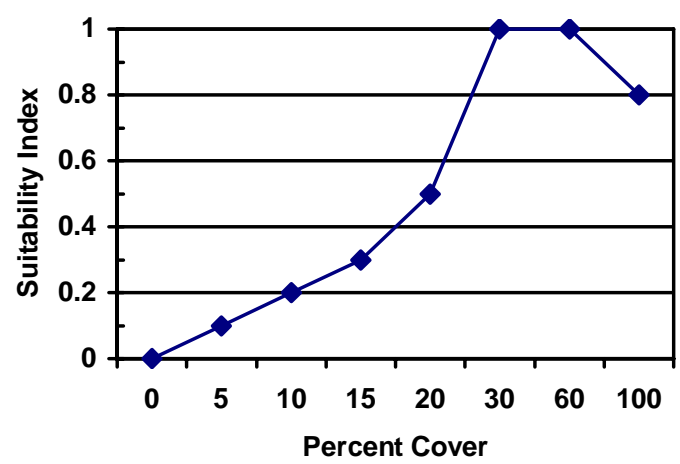

V2: Percent cover all shrubs $<5 \mathrm{ft}$ in height

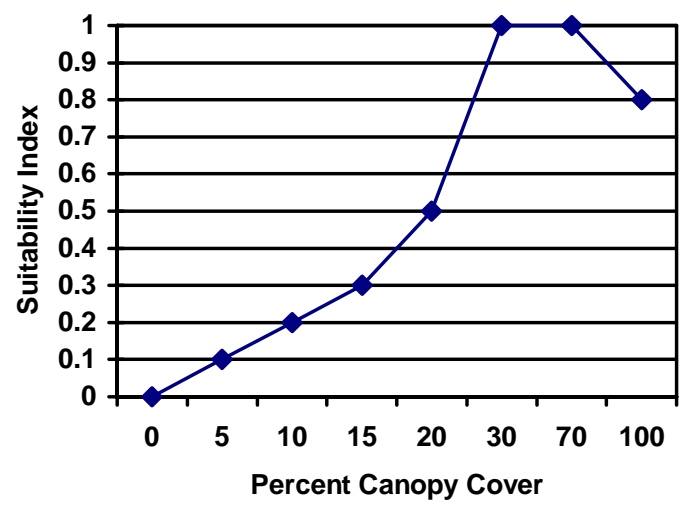

V3: Mean shrub height

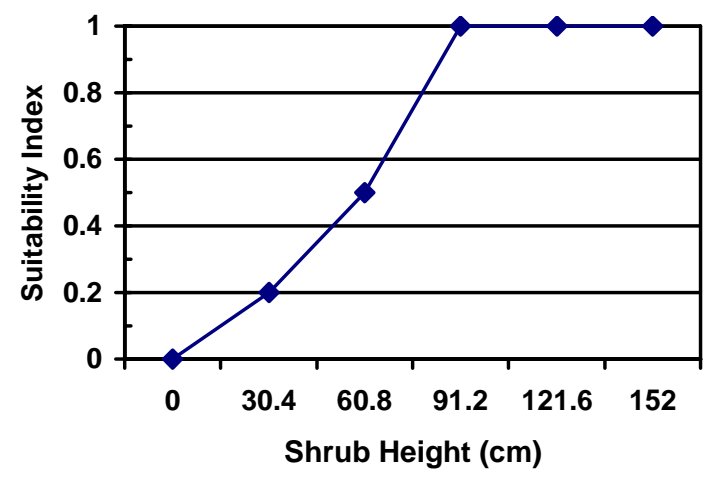


V4: No. of preferred shrub species

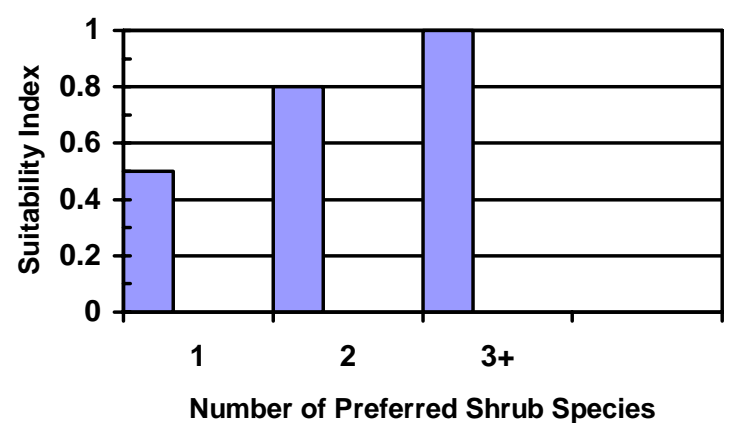

V5: Percent cover palatable herbaceous species

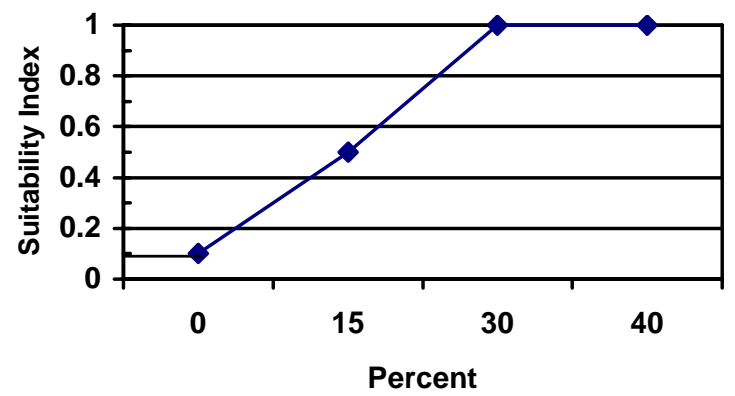

V6: Presence of suitable agricultural crops within 1.6 kilometers (1 mile) of study area Yes: 0.1

No: 0.0 
V7: Aspect

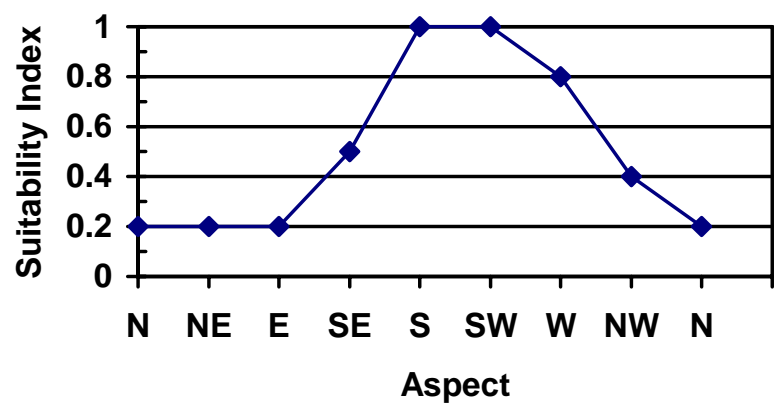

V8: Road density

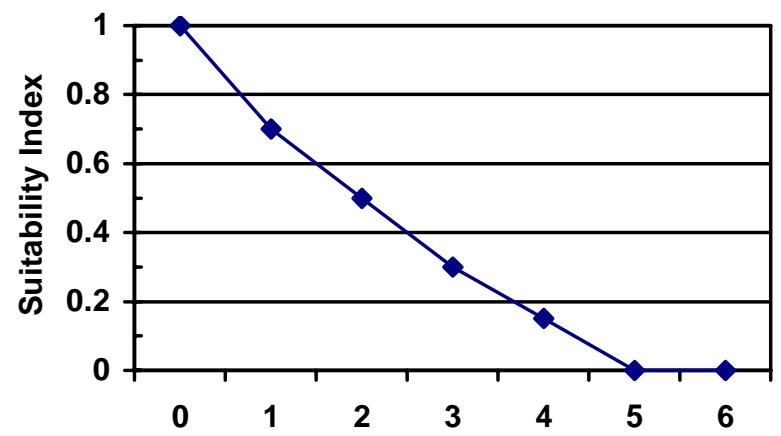

Kilometers of Open Road per Square Kilometer

V9: Topographic diversity

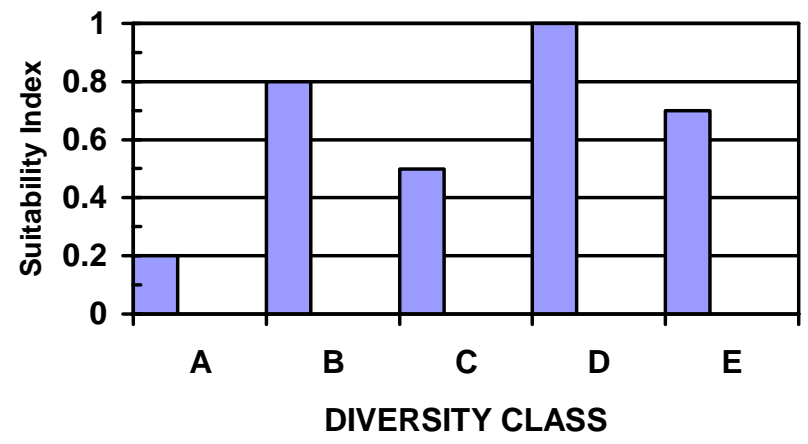


Eder Acquisition 2007 HEP Report

$\mathrm{V}_{9} \quad$ Topographic diversity.

A: Level terrain less than 5 percent slope.

B: Level terrain broken by drainages.

C: Rolling terrain 5 to 25 percent slope.

D: Rolling terrain with rims, ridges, and/or drainages.

E: Mountainous terrain with slopes greater than 25 percent.

V10: Percent evergreen cover $>5 \mathrm{ft}$ in height

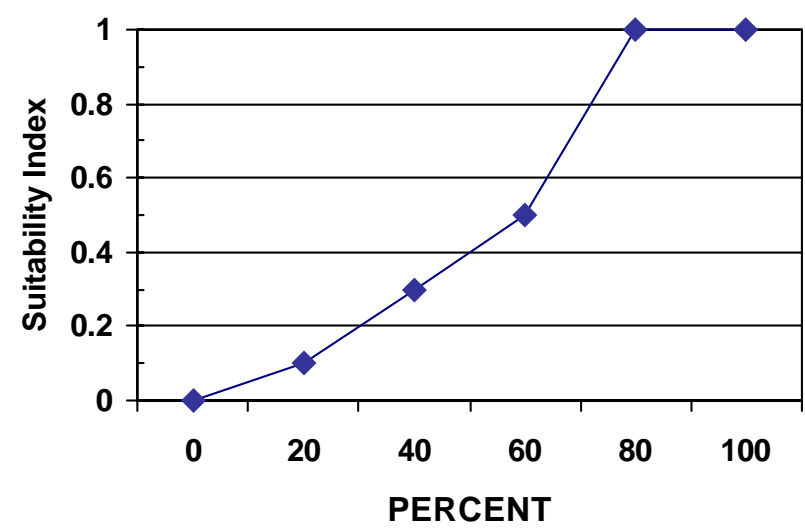

Shrubsteppe $\mathrm{HSI}=$ minimum value $\mathrm{WFI}$ or $\mathrm{WCI}$

$\mathrm{WFI}=(((\mathrm{V} 1(\mathrm{~V} 2 \times \mathrm{V3} \times \mathrm{V4} \times \mathrm{V5}) \mathbf{1 / 4})+\mathrm{V6}) \times \mathrm{V7})^{\wedge .625} \times \mathrm{V8}$

Steps in calculating WFI with a hand calculator:

1. Obtain geometric mean of V2, V3, V4, and V5

2. Multiply product from step one by V1 and add V6

3. Multiply sum obtained in step two by V7

4. Take the 1.66 root $(\wedge .6$ on your computer)of product from step 3

5. Multiply result from step 4 by V8 to obtain WFI

$\mathrm{WCI}_{\mathrm{SS}}=(\mathrm{V9} \times \mathrm{.8})+\mathrm{V} 10$

Conifer Forest HSI = Lower Value Between:

WFI $=(((V 1(V 2 \times V 3 \times V 4 \times V 5) ~ 1 / 4)+V 6) \times V 7)^{\wedge .625} \times$ V8

$\mathrm{WCI}_{\mathrm{F}}=2(\mathrm{V10})+\mathrm{V} 9$ 
Eder Acquisition 2007 HEP Report

\section{Sharp-tailed Grouse}

V1: Mean VOR - Landscape (all vegetation including residual)

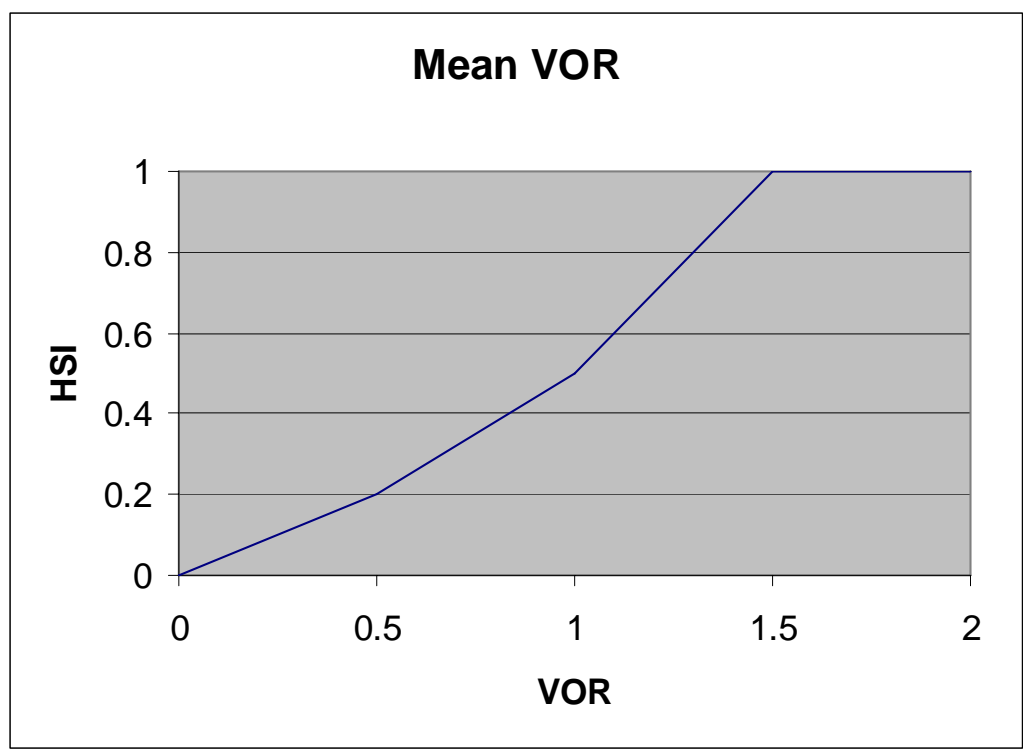

V2: Percent Slope

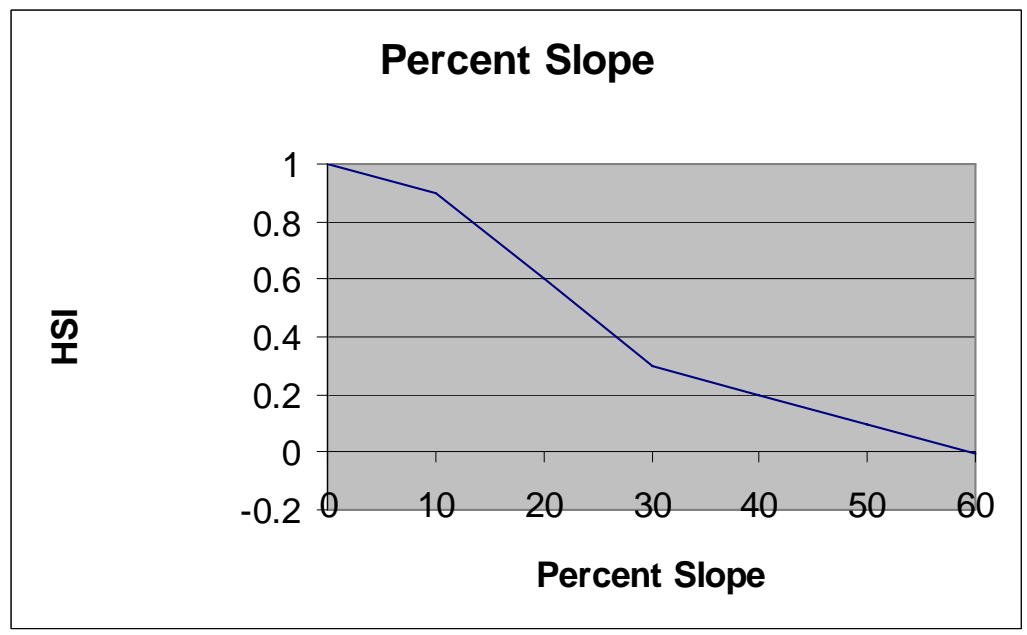

Nesting Habitat HSI Equation: $($ V1 x V2 x V6) 
Eder Acquisition 2007 HEP Report

Brood Rearing Habitat

V3: Percent Cover Grass

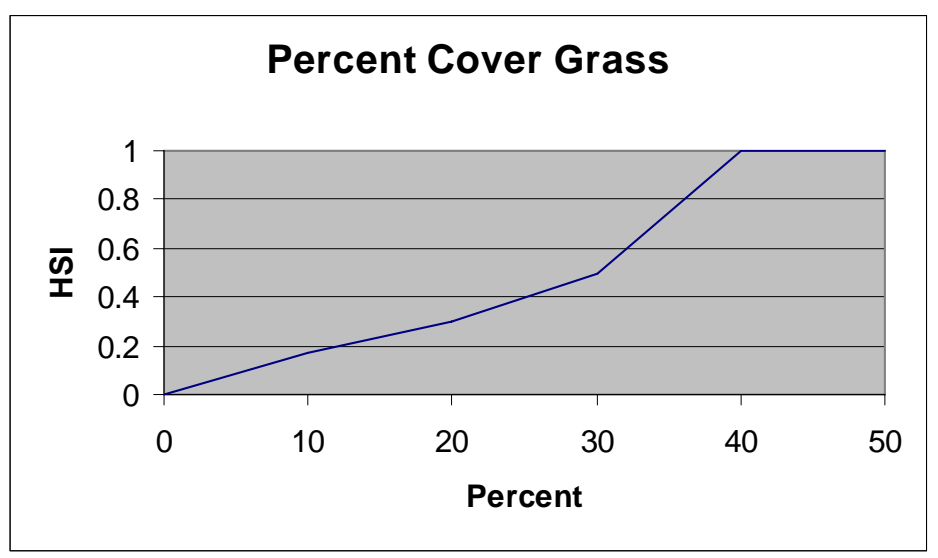

V4: Percent Cover Forbs

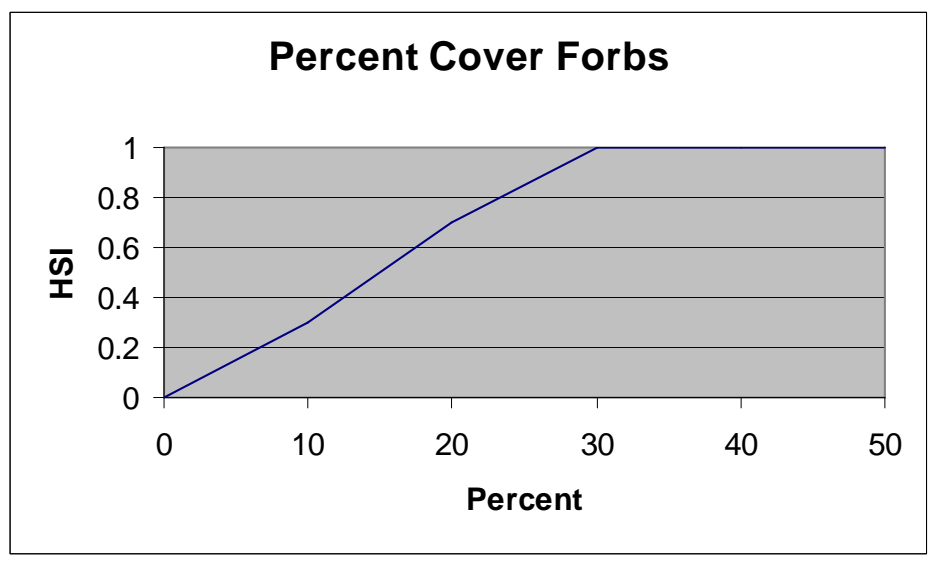

V5: Percent Cover Introduced Herbaceous Species

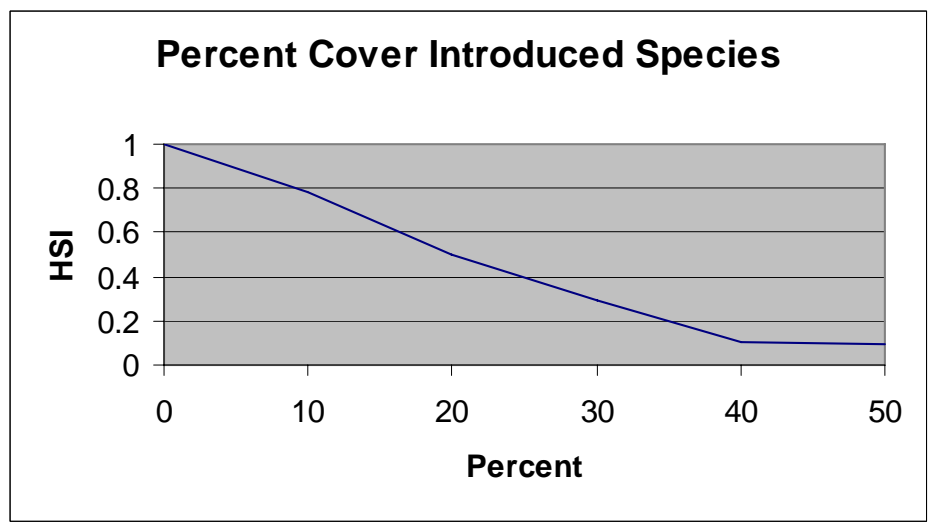


Eder Acquisition 2007 HEP Report

V7: Distance Between Nesting/Brood Rearing and Winter Habitat

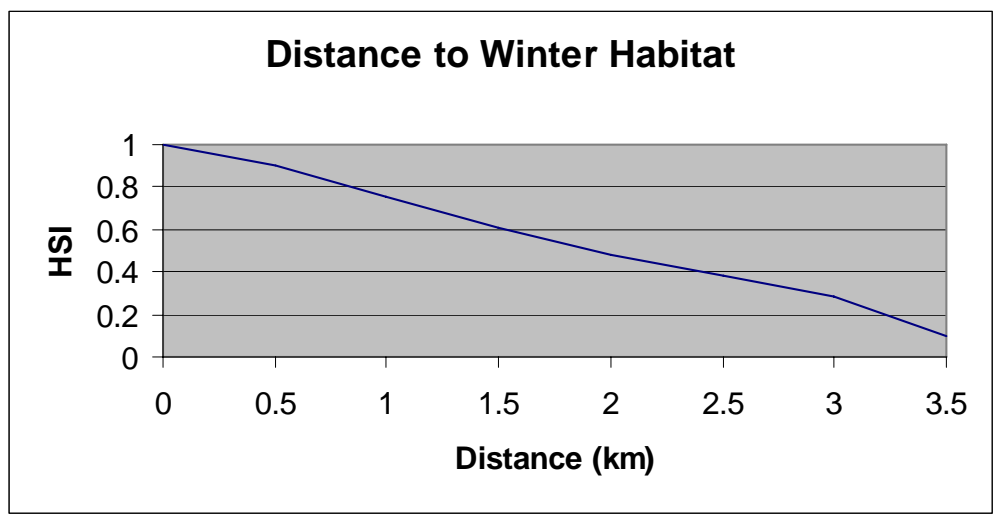

Brood Rearing HSI Equation: $\left[[((\mathrm{V3}+\mathrm{V4}) / 2)(\mathrm{V} 6)(\mathrm{V} 7)]^{1 / 3}(\mathrm{~V} 5)\right]$

Nesting/Brood Rearing HSI $=(\text { Nesting HSI } x \text { Brood Rearing HSI })^{1 / 2}$

\section{Winter Habitat}

V8: Percent Cover Deciduous Shrubs and Trees

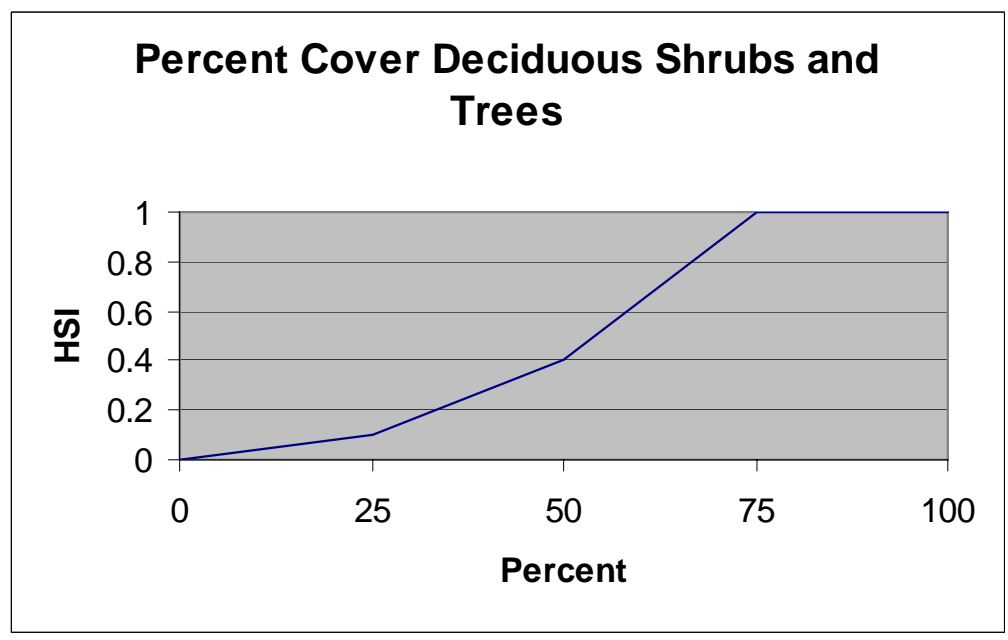

V9: Deciduous Shrub and Tree Composition/Wheat Availability

\begin{tabular}{|c|c|c|}
\hline Attribute & Species & SI \\
\hline Upper Canopy & Water Birch, Aspen, Cottonwood & 0.5 \\
\hline Mid Canopy & $\begin{array}{c}\text { Serviceberry, Hawthorn, } \\
\text { Chokecherry }\end{array}$ & 0.3 \\
\hline Lower canopy & Rose, Snowberry & 0.2 \\
\hline $\begin{array}{c}\text { Agricultural } \\
\text { Fields }\end{array}$ & Standing Wheat or Wheat Stubble & 0.2 \\
\hline HSI & Additive : Not to exceed 1.0 & $1.2=$ \\
& & 1.0 \\
\hline
\end{tabular}


Eder Acquisition 2007 HEP Report

V10: Percent Area Providing Winter Habitat

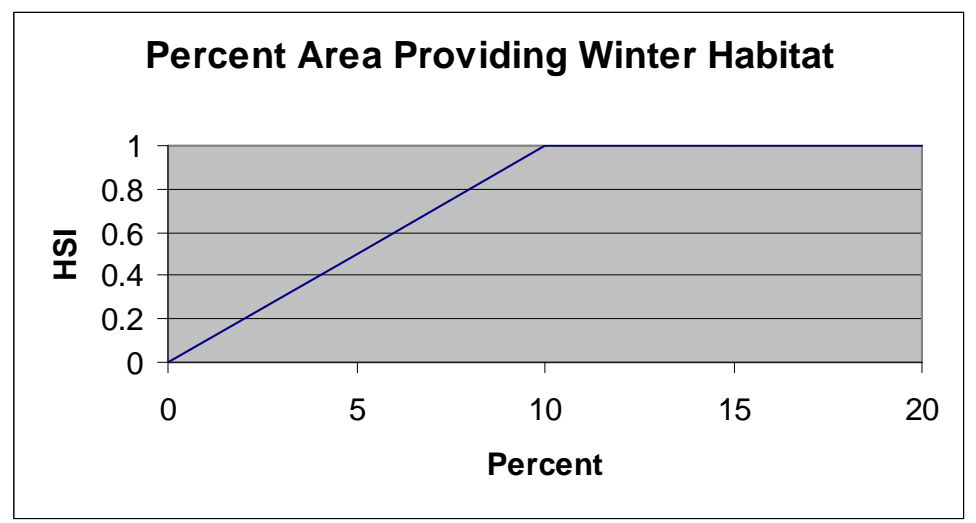

Winter HSI Equation: $\left((\mathrm{V} 8 \times \mathrm{V9})^{1 / 2} \times \mathrm{V10}\right)$

Model HSI: Consists of two HSI's: Nesting/Brood Rearing HSI and Winter HSI.

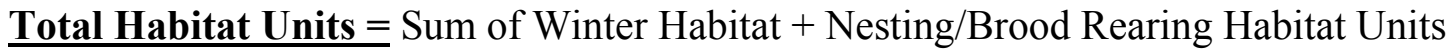




\section{Western Meadowlark}

\section{WESTERN MEADOWLARK}

Modified from Schroeder and Sousa, 1982.

Cover Types: Grassland, Shrubgrass, Shrubland, Pasture, Shrub-steppe

V1: Percent canopy cover of herbaceous plants

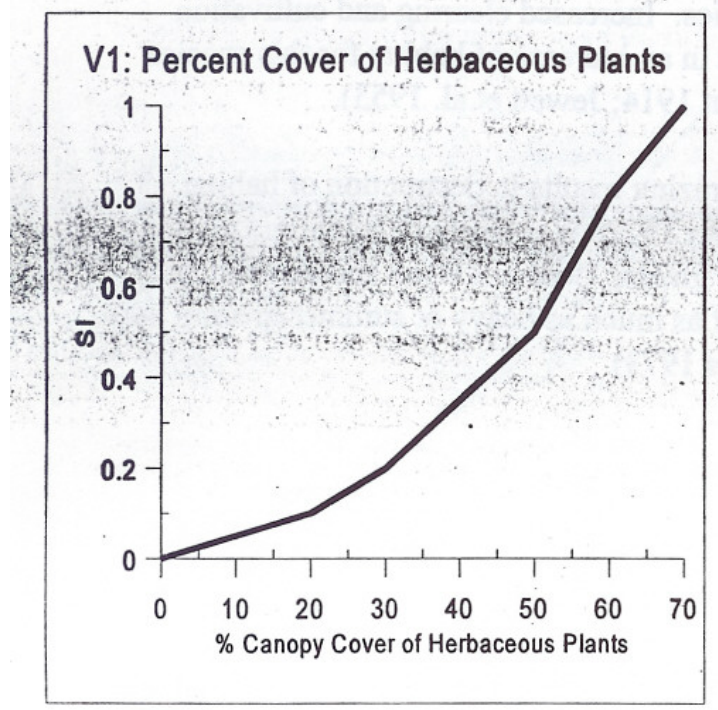

\section{Cover Requirements}

Western meadowlarks are adapted to short grass and mixed grass prairies, preferring large fields with short vegetation and good drainage. Western meadowlarks exhibit tolerance for a wide variety of plant associations and are widely distributed in Washington-commonly occurring in meadows, orchards, thickets, and cultivated areas. Conversion of woodlands to agricultural fields has favored western meadowlark populations in Washington.

V2: Percent of herbaceous canopy cover composed of grass

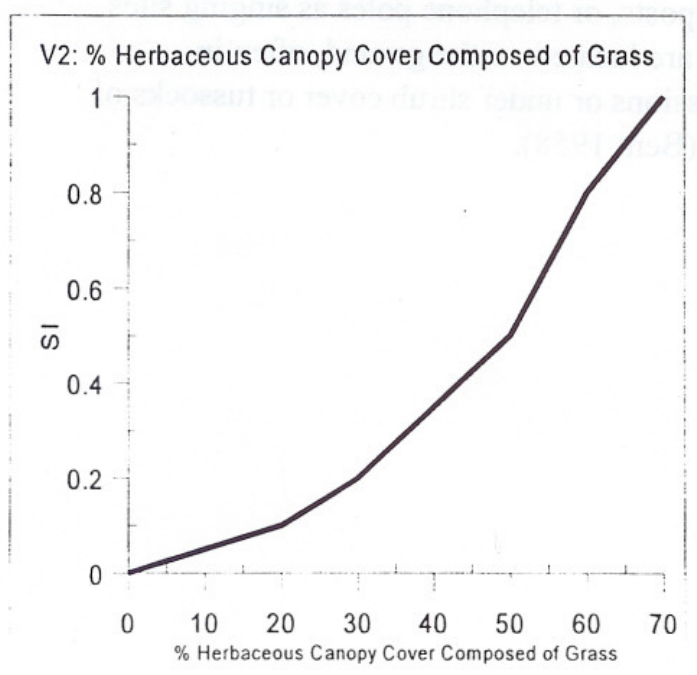

\section{Food Requirements}

Bryant (1914), Weins (1973) et.al. suggest that animal material, primarily insects, comprise approximately $63 \%$ of the meadowlark's diet while $37 \%$ is made up of vegetative matter. Vegetable matter consisted of one-third grain and two-thirds weed seeds. Spring and summer diet was primarily insects with a shift to seeds in fall and winter. Hubbard and Hubbard (1969) reported meadowlarks eating carrion including their own species. It is doubtful that food supply is ever a limiting factor for this species (Lanyon, 1956).

Water Reauirements 
Eder Acquisition 2007 HEP Report

V3: Average height of herbaceous canopy (inches)

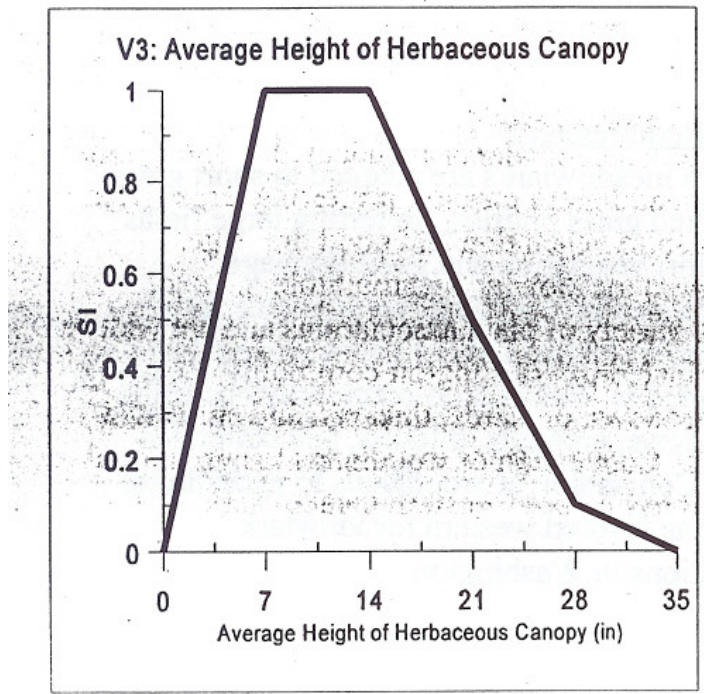

V4: Distance to Perch Sites (feet)

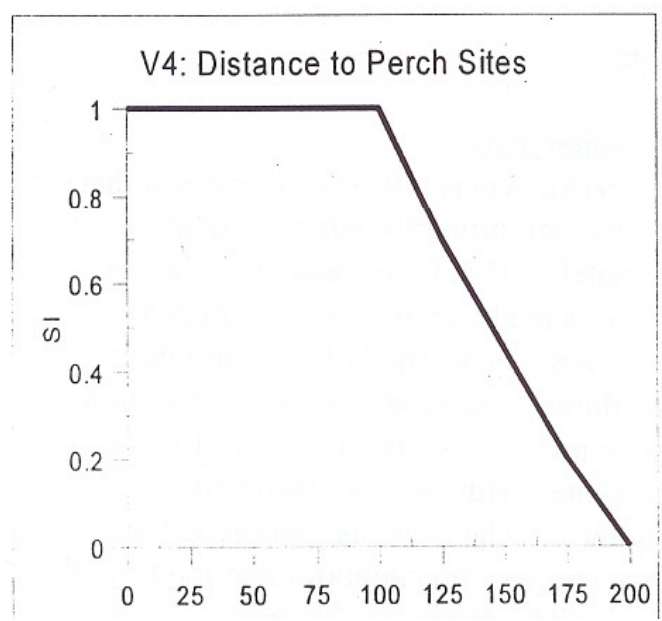

Because of its habitat preferences, western meadowlarks are affected by agricultural activities. Increased clearing and cultivation results in an increase of habitat for this species (Bryant 1914; Jewett et.al. 1953). Overgrazing results in destruction of habitat (Rohwer 1972. Weins 1973). Light grazing or winter grazing does not affect meadowlark habitat as much as heavy or summer grazing (Weins 1973).

Reproductive Requirements Males require elevated perches, such as shrubs, fence posts, or telephone poles as singing sites. Nests are located on the ground, often in depressions or under shrub cover or tussocks of grass (Bent 1958). 
Eder Acquisition 2007 HEP Report

V5: Percent Shrub Canopy Cover

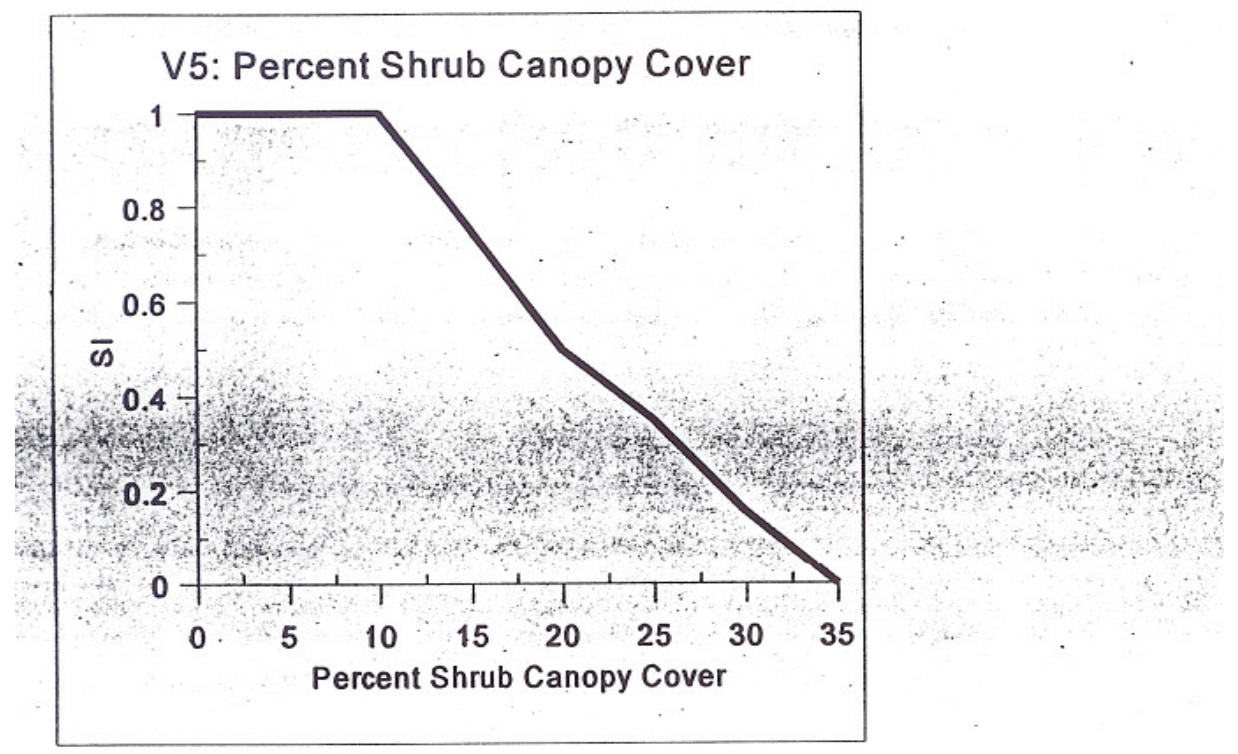

Model Equation:

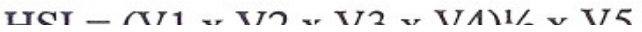

$\mathrm{HSI}=(\mathrm{V} 1 \times \mathrm{V} 2 \times \mathrm{V} 3 \times \mathrm{V} 4)^{1 / 2} \times \mathrm{V} 5$ 
Eder Acquisition 2007 HEP Report

\section{Bobcat}

Variable 4. Percent of area comprised of rockpiles, rock outcrops, rocky ledges, boulder fields, talus slopes and cliffs [include only tops and bottoms of cliffs and not cliff faces (pers comm., Steve Knick)].

Assumes:

(1) Bobcats prefer rocky or broken terrain.

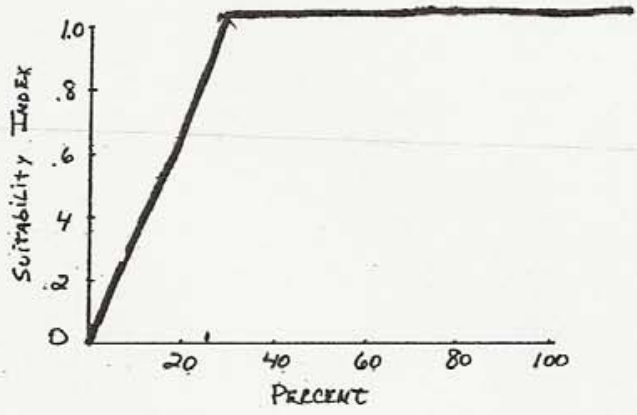

Model Relationships

In order to calculate suitability indices for food and for cover, the variables for each life requisite were combined into an equation. Because food requirements and cover/reproductive requirements are of equal importance, the SI's were derived to express each life requisite as separate values for the overall HSI determination (see below).

Suitability Indices

Food

$$
\mathrm{SI}_{f}=\frac{\mathrm{V} 1+2 \mathrm{~V} 2}{3}
$$

Cover/reproduction

$$
\mathrm{SI}_{\mathrm{c} / \mathrm{r}}=\frac{\mathrm{V} 3+2 \mathrm{~V} 4}{3}
$$


Eder Acquisition 2007 HEP Report

\section{Downy Woodpecker}

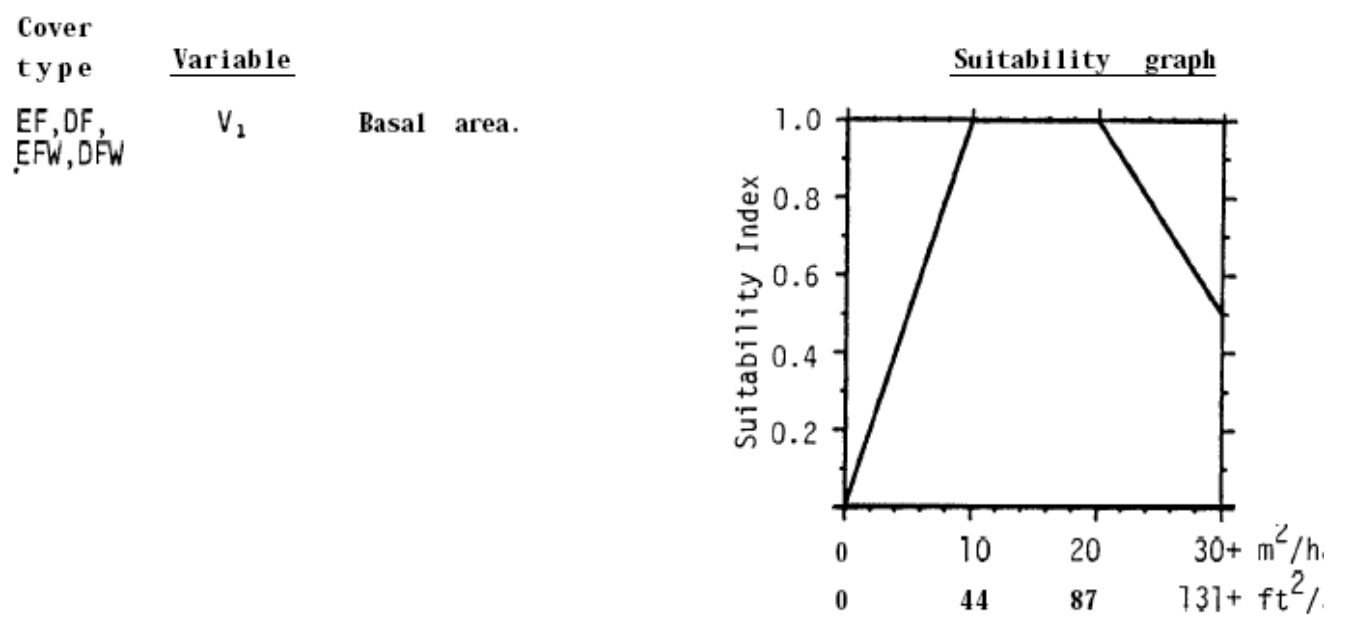

$\begin{array}{lll}E F, D F, & V_{2} & \text { Number of snags } \\ \text { EFW, DFW } & >15 \mathrm{~cm} \mathrm{dbh} / 0.4 \mathrm{ha} \\ & (>6 \text { inches dbh/ } \\ & 1.0 \text { acre }) .\end{array}$

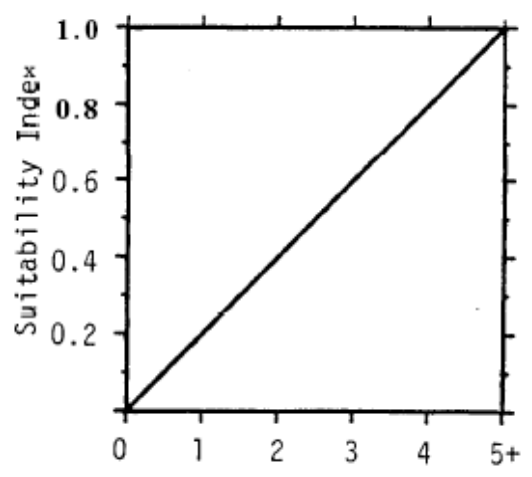

Life requisite values. The life requisite values for the downy woodpecker are presented below. 
Eder Acquisition 2007 HEP Report

Life requisite

Food

Reproduction

\section{Cover type}

EF , DF , EFW , DFW

EF , DF , EFW , DFW
Life requisite value<smiles>[AlH2]</smiles>

$V_{2}$

HSI determination. The HSI for the downy woodpecker is equal to the lowest life requisite value.

Application of the Model

Definitions of variables and suggested field measurement techniques (Hays et al. 1981) are provided in Figure 2 .

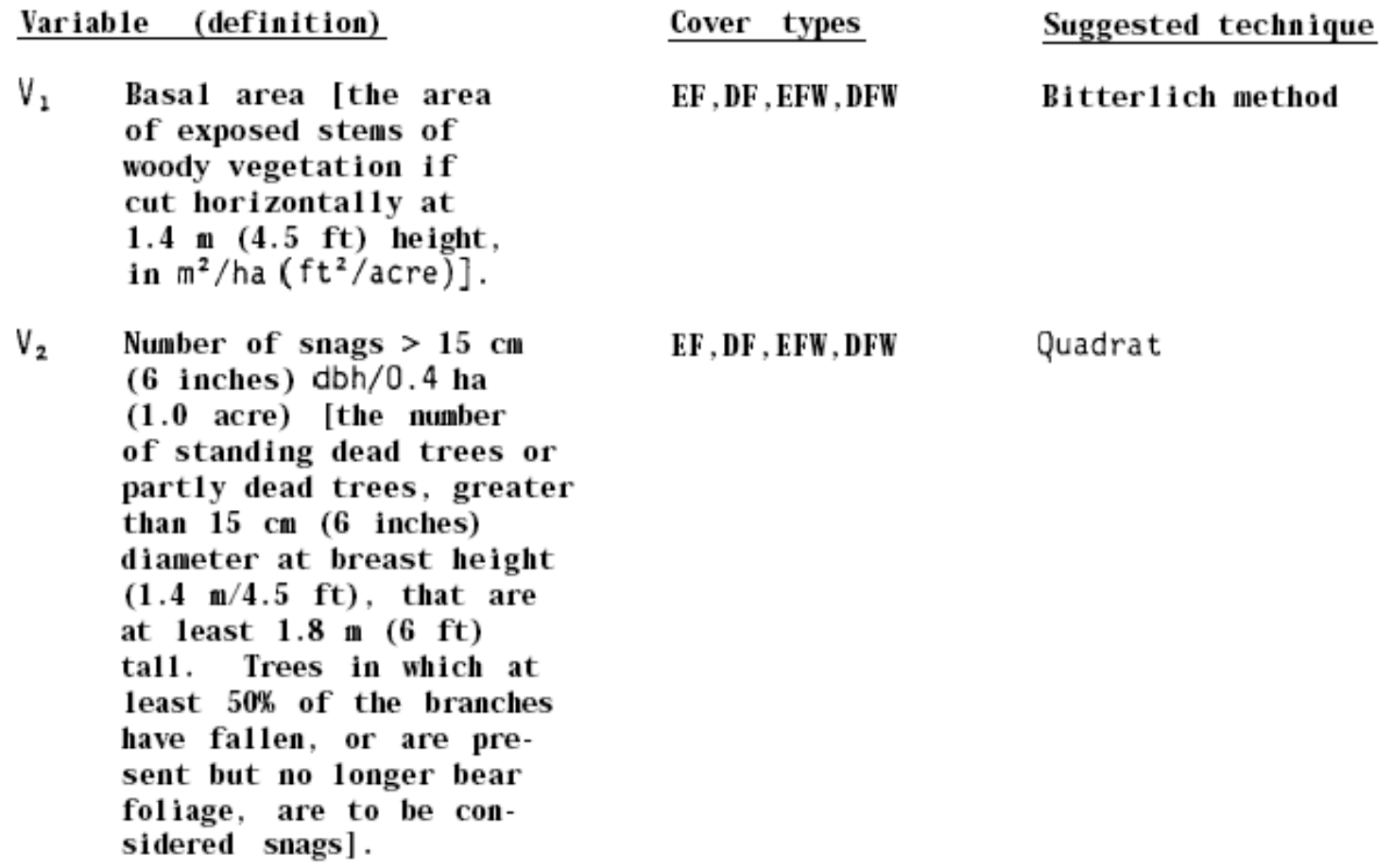




\title{
Appendix B - Measurement Protocols
}

\author{
HABITAT EVALUATION PROCEDURES
}

STANDARD MEASUREMENT PROTOCOLS AND TECHNIQUES (Draft)

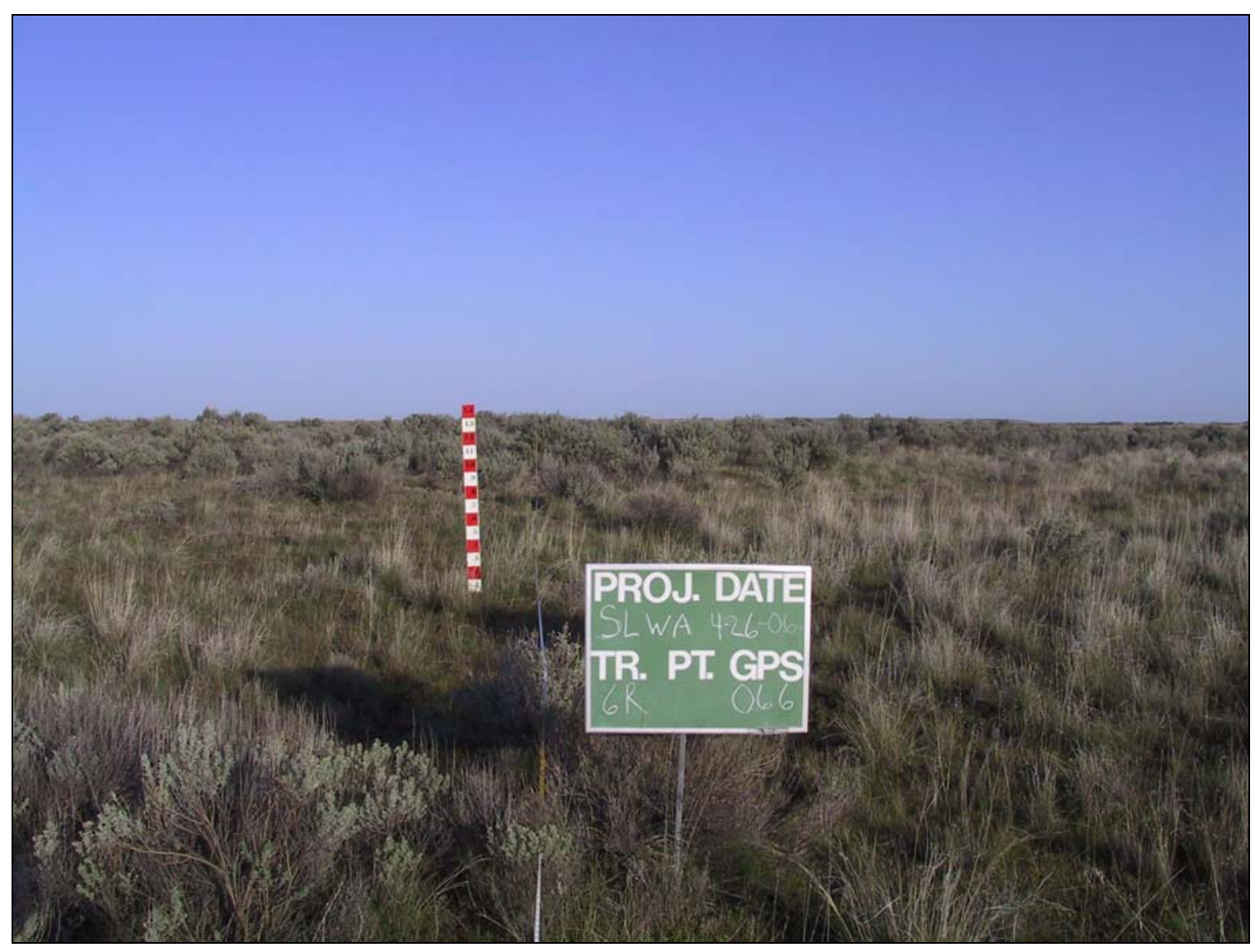

Compiled By

Paul R Ashley - RHT Coordinator

November 2006 
Eder Acquisition 2007 HEP Report

\section{HEP Sampling Design and Measurement Protocols}

\section{Introduction}

This document was developed to fulfill a request by the Upper Columbia United Tribes (UCUT) and Bonneville Power Administration (BPA) to develop a "stand alone" reference for Habitat Evaluation Procedures (HEP) transect protocols used by the Regional HEP Team (RHT). General and specific protocols are described. General protocols include a brief description of pre HEP survey pilot studies; transect establishment guidelines, and photo documentation parameters. In contrast, specific metrics detail actual habitat variable measurement techniques including diagrams where additional explanation is needed.

Specific metrics are identified with an alpha-numeric code. This allows project managers and others to identify specific measurement techniques in report tables without lengthy, redundant explanations. This report is intended to be a "living" document and will be modified as needed. The following standardized protocols and measurement techniques are used by the Regional HEP team to measure habitat variables described in HEP models.

\section{General Protocols}

\section{Pilot Studies}

Pilot studies are conducted in new habitat types and/or familiar habitat types that are comprised of unique structural conditions/key ecological correlates. Pilot study data is used to estimate the sample size needed for a confidence level $\geq 80 \%$ with a $10 \%$ tolerable error level (Avery 1994) and to determine the most appropriate sampling unit ${ }^{5}$ for the habitat variable of interest i.e., a coefficient of variation analysis (BLM 1998). In addition, a power analysis is conducted on pilot study data (and periodically throughout data collection) to ensure that sample sizes are sufficient to identify a minimal detectable change of $20 \%$ in the variable of interest with a Type I error rate $\leq 0.10$ and $\mathrm{P}=0.9$ (BLM 1998, Block et al. 2001). All field data is recorded on data loggers or data sheets and downloaded/transferred to data summary spreadsheets.

Transects

Transect cover sheets are used to document specific transect information including transect identification, cover type, HEP Team members, global positioning system (GPS) coordinates, and other pertinent information.

Transects are established at least 300 feet (100 meters), where possible, from ecotones, roads, and other anthropogenic influences. Transect starting points and azimuths (direction) are randomly selected for each cover type. Start points are selected based on superimposing a UTM grid over cover type maps and identifying specific X/Y coordinates with the aid of a random numbers table, or computer generated random number generator/point locater program.

\footnotetext{
${ }^{5}$ Includes micro-plot grid size and shape etc.
} 
Transect start, turn, and end points are marked with 14 -inch (36 centimeter) 0.25 inch ( 0.6 centimeter) diameter rebar stakes ${ }^{6}$ painted fluorescent orange or red. GPS positions (UTM coordinates-NAD 27) are recorded at start, turn, and end points. If cover types change or transect length is greater than 300 feet, another transect azimuth is randomly selected, or the original azimuth is varied by 45 degrees (direction [left or right] is determined by the flip of a coin where more than one choice is possible). Compass azimuths (headings) are magnetic bearings i.e., not corrected for local declination. Transects are divided into 100 foot (30 meter) sample units for statistical purposes.

\section{Photo Points}

Photo points are established at the start point of each transect. Pictures are recorded from a height of three feet at the beginning of each transect while facing in the direction of the transect azimuth. A transect reference board (includes transect number, project name, date, GPS reference number) is placed at the 15 foot interval while a cover board is placed at the 30 foot mark on each transect. Occasionally, panoramic photographs are also needed e.g., dense vegetation, linear/narrow cover types. Habitat conditions are photographed with a Canon G1 ${ }^{\circledR} 3.3$ mega pixal digital camera (with and without magnification).

\section{Specific Metrics}

Metrics generally follow those described by Hays et al. (1981) and/or Avery (1994) unless otherwise noted. Some metrics have been modified due to extreme field conditions and/or to better meet Regional HEP Team needs.

\section{Herbaceous Measurements}

\section{Percent Cover}

1. Herbaceous percent cover measurements are recorded at 20 or 25 -foot intervals on the right side of the transect tape (the right side is determined by standing at 0 feet and facing the line of travel/transect azimuth). RHT members walk on the left side of the transect line to reduce sample disturbance. A square $0.1 \mathrm{~m}^{2}$ micro-plot grid is used in grasslands to estimate percent cover of herbaceous vegetation while a rectangular $0.5 \mathrm{~m}^{2}$ grid is generally used in shrublands (the $0.5 \mathrm{~m}^{2}$ grid may also be used in grasslands if desired). The near right hand corner of the grid is placed at the sampling interval (rectangle grids are placed with the long axis perpendicular to the tape, and the lower right corner on the sampling interval). An example of micro-plot grid placement is shown in Figure 1 . Approximately $20 \%$ of the micro plot is covered by vegetation in the example. Grid samples are considered independent samples for statistical purposes.

1A: $0.1 \mathrm{~m}^{2}$ micro-plot grid/20' interval

\footnotetext{
${ }^{6}$ Marking transect points with rebar stakes is at the discretion of the project proponent. Therefore, not all transects are marked in this manner.
} 
1B: $0.1 \mathrm{~m}^{2}$ micro-plot grid/25' interval

$1 \mathrm{C}: 0.5 \mathrm{~m}^{2}$ micro-plot grid/20' interval

1D: $0.5 \mathrm{~m}^{2}$ micro-plot grid $/ 25$ ' interval

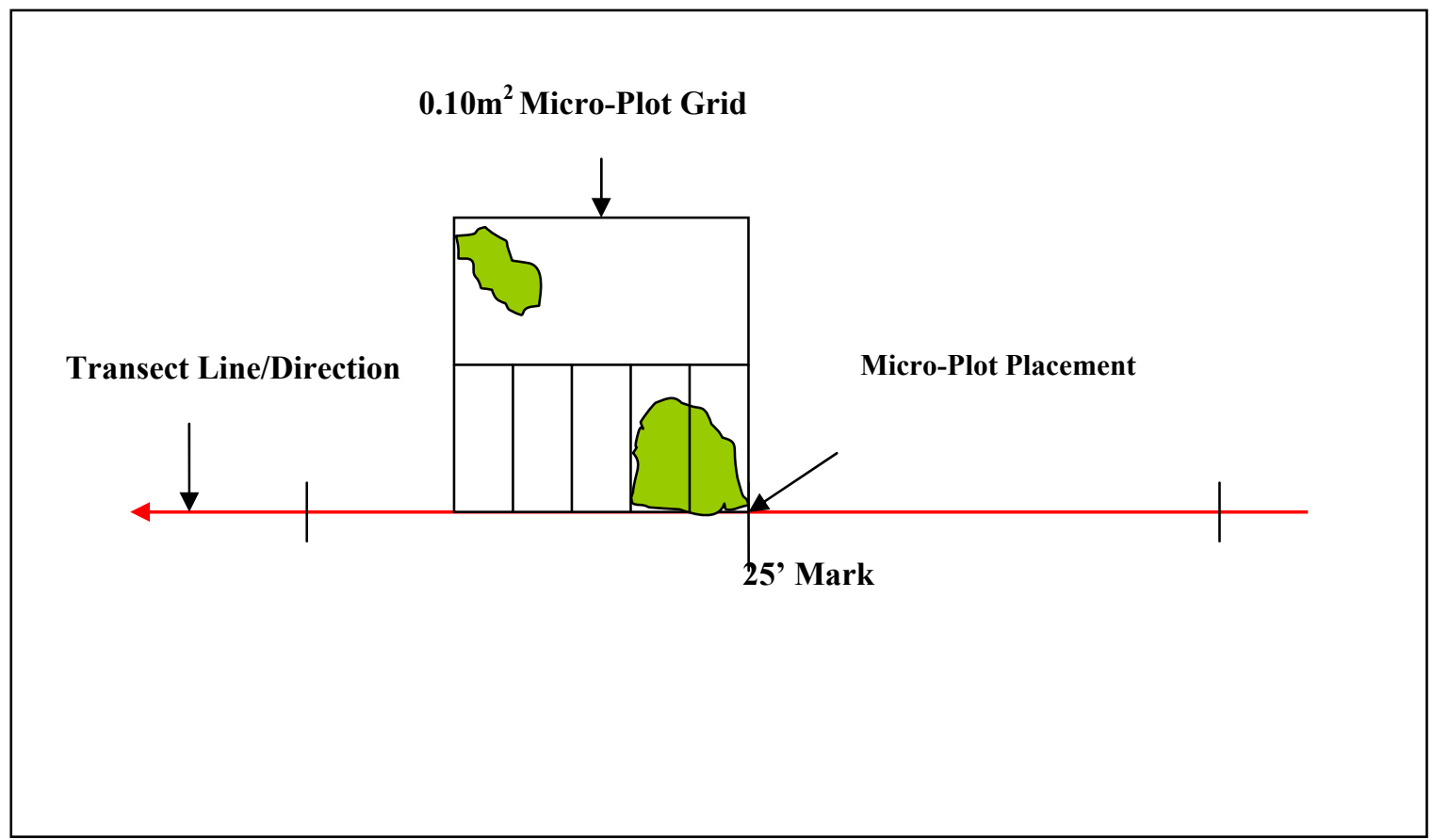

Figure 1. Micro-plot grid placement and percent cover example.

\section{Height}

2. Herbaceous height is measured with a measuring rod placed within the grid frame $($ scale $=10$ ths $/ \mathrm{ft}$.). Three evenly spaced measurements are recorded and averaged for each sample. Only leaf material is measured (leaves provide the greatest amount of cover). "Leaf material" may include residual cover and/or new growth predicated on HEP model variable requirements. Grass inflorescence is not included in height measurements.

2A. Four measurements, one from each corner of the micro plot grid, are recorded and averaged for each sample. Only leaf material is measured (leaves provide the greatest amount of cover). Grass inflorescence is not included in height measurements.

2B. A measuring rod is held vertical at the interval point: the highest vegetation to cross the measuring rod at that point is measured to the nearest tenth of a foot.

2B-1: 10' interval

2B-2: 20' interval

2B-3: 25 ' interval

Visual Obstruction Readings (VOR) 
3. A Robel pole (Robel 1975) is used to document vertical and/or horizontal cover for herbaceous vegetation i.e., visual obstruction readings (VOR). Measurements are recorded at 20,25, or 50-foot intervals. Intervals are determined by the length of each transect, i.e., a minimum of 12 measurements are required for each transect, or cover type heterogeneity (structurally diverse cover types generally require larger sample sizes).

The Robel pole (Robel 1975) is placed on the transect line at the appropriate interval. Four observations are taken from a distance of four meters from the Robel pole and averaged to obtain a single visual obstruction reading or VOR. Observers sight over a one meter pole and record how much of the Robel pole is totally obscured from the ground up (Figure 2). Measurements are reported in 0.25 decimeter increments.

Two measurements are taken on the transect line on opposite sides of the Robel pole; two identical measurements are taken from the same point perpendicular to the transect line for a total of four "readings" (Figure 3). Sample size is determined to be adequate when the "running mean" varies $\leq 10 \%$ of the mean. VOR samples are considered independent for statistical purposes.

3A: 20 ' interval

3B: 25 ' interval

$3 \mathrm{C}$ : 50 ' interval

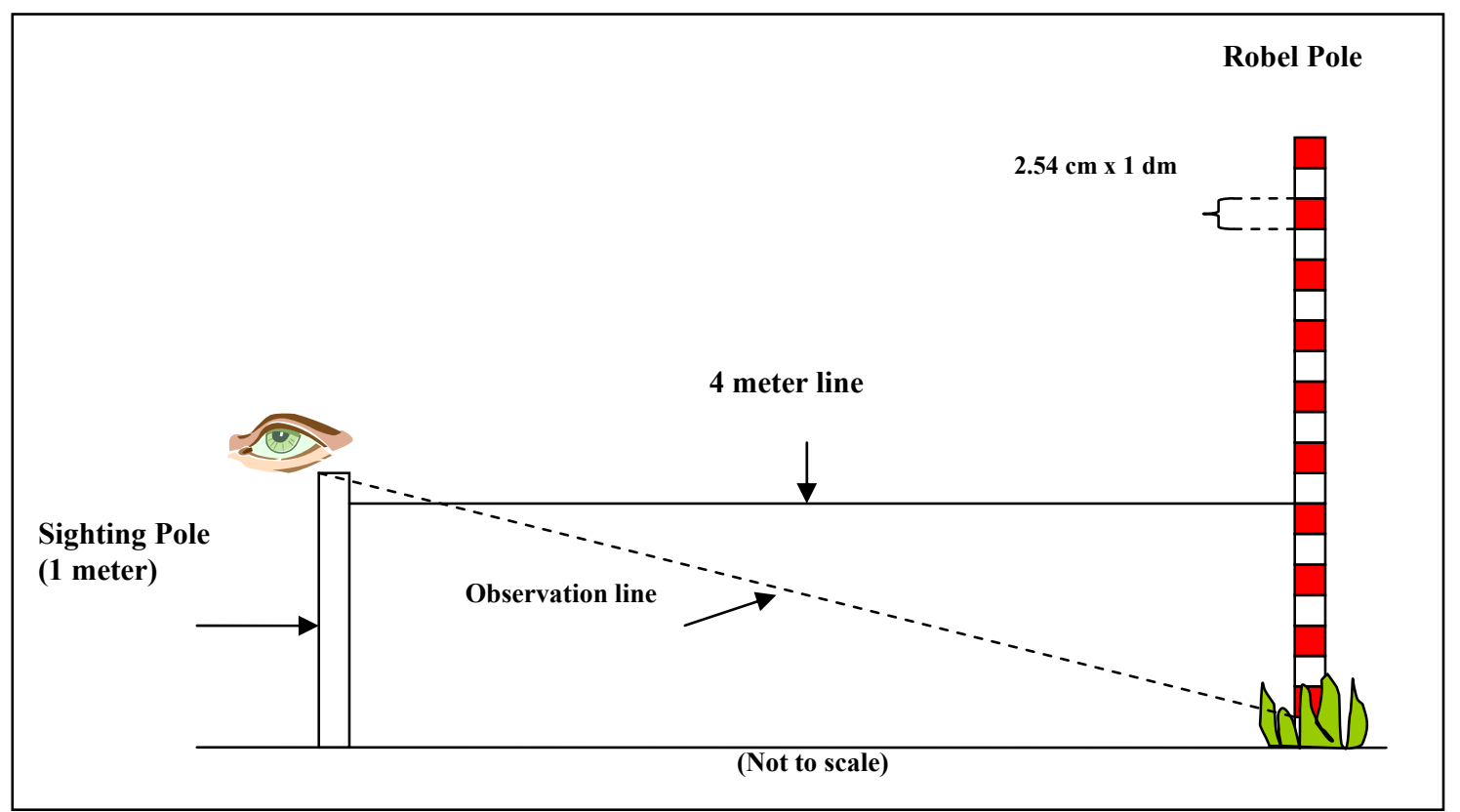

Figure 2. Visual obstruction reading diagram. 


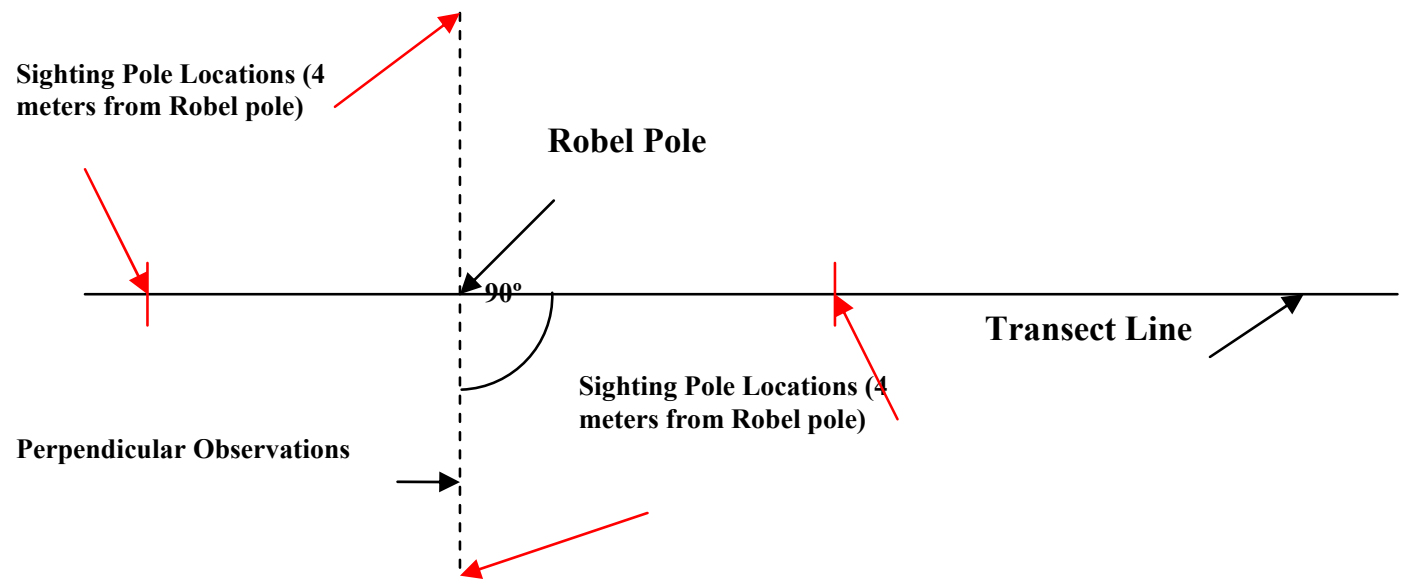

("Birds eye" View)

Figure 3. Robel pole "readings" layout diagram.

\section{Shrub Measurements}

\section{Percent Cover}

4. Line intercept or point intercept (USFWS 1981) is used to determine shrub cover. Line intercept is generally used when shrub cover is estimated at $<5 \%$ (the most accurate results are obtained using the line intercept method). In contrast, the point intercept method is used if shrub cover is estimated at $>5 \%$.

4A: Line intercept is used to measure the amount of cover that intercepts the transect line as illustrated by the red lines shown in Figure 4. Measurements are in $10^{\text {ths }}$ of feet. Gaps in vegetation less than four tenths of a foot (5 inches) are ignored. The amount covered by shrubs is added to determine shrub intercept for each transect. For example, if 7.5 feet of a 100 -foot long transect is covered by shrubs, percent cover is $7.5 \%$.

Shrub cover is recorded by species. Where shrubs overlap, shrub intercept is recorded for the tallest shrub and noted for the lower shrub(s). 


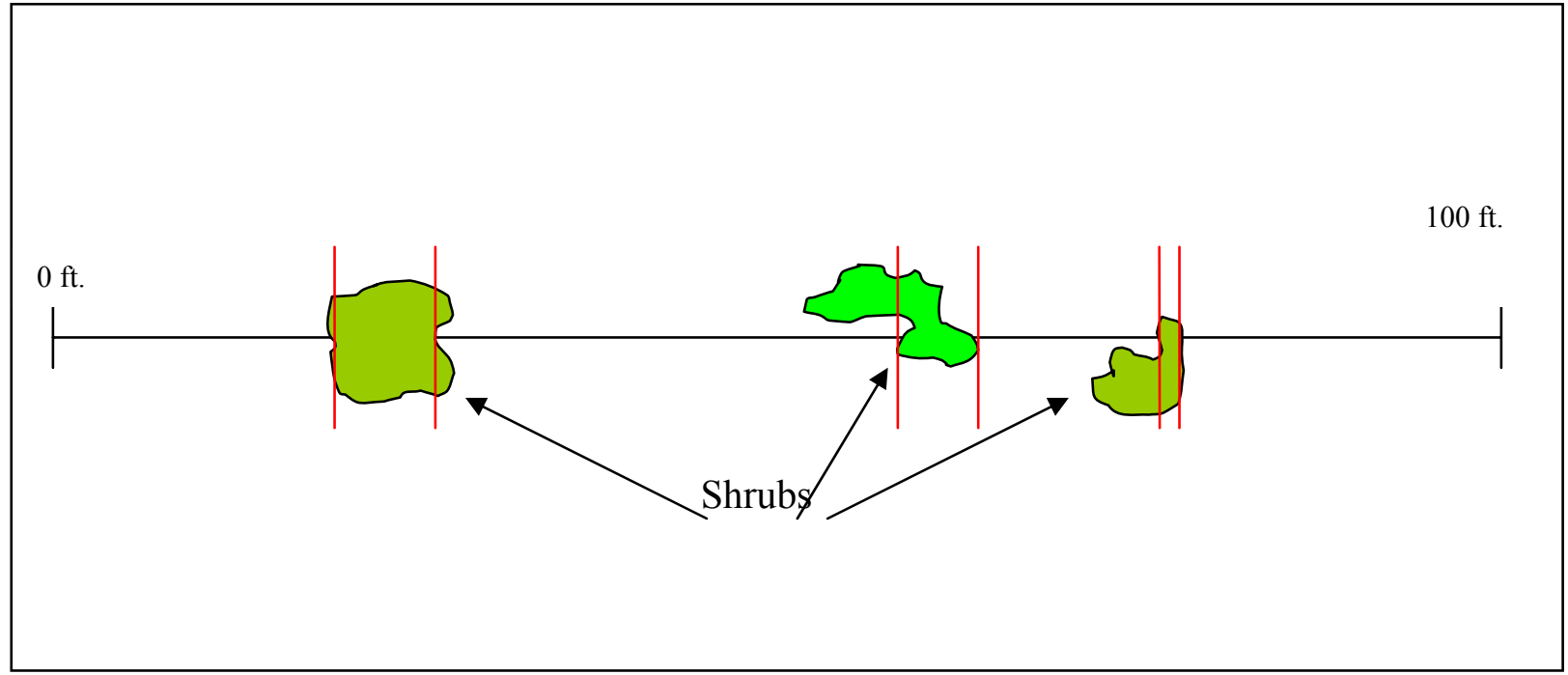

Figure 4. Line intercept method example.

4B: Point intercept is used when shrub canopy cover is estimated at $\geq 5 \%$. Shrub cover is determined by recording the number of "hits" at specific intervals along a transect line. To be counted as a "hit", a portion of the shrub must cross the transect tape's interval number line e.g., 2', 4', 6'... nth. If a portion of the shrub does not break the vertical plane at the interval number line, it is reported as a miss (Figure 5). Either a "hit" or "miss" is recorded on data loggers and/or paper data sheets for each designated interval.

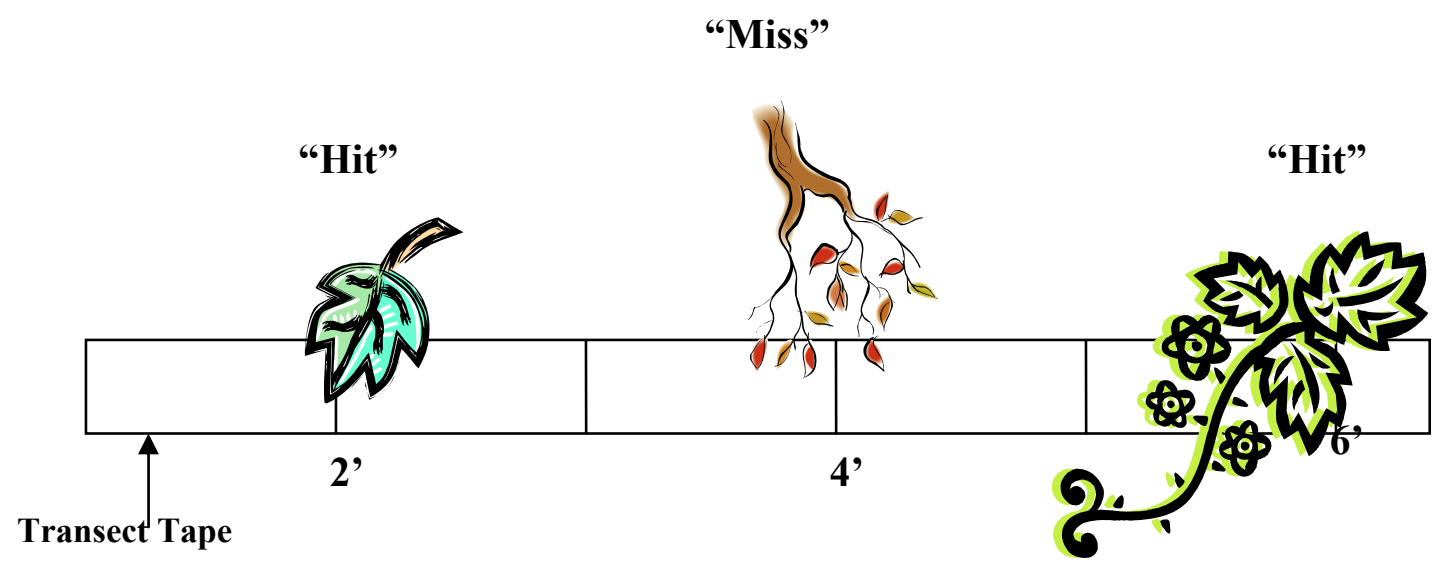

Figure 5. Point intercept method example showing "hits" and "misses" at two foot intervals. 
From $5 \%$ to $20 \%$ cover, point data is collected at two-foot intervals ( 50 possible "hits" per $100 \mathrm{ft}$. sample unit). If shrub cover is estimated at $>20 \%$, shrub point data is collected at five foot intervals (20 possible "hits" per 100 $\mathrm{ft}$. sample unit). On rare occasions, ten-foot intervals may be used when shrub cover exceeds 50\% (10 possible "hits" per $100 \mathrm{ft}$. sample unit). The ten-foot interval is generally applied to shrub monocultures, or areas with few shrub species that exhibit relatively equal shrub distribution/density.

Shrub "hits" are recorded by species. Where shrubs overlap, shrub intercept is recorded for the tallest shrub and noted for the lower shrub(s).

4B-1: 2' interval

4B-2: 5 ' interval

4B-3: 10' interval

4C: Modified point method is used when shrub cover is impenetrable or otherwise inaccessible. A baseline transect is established along the shrub edge. A six-foot measuring rod is then inserted into the shrub cover at right angles to the baseline tape at appropriate intervals. Recorders estimate shrub "hits", species information, and height data where the end of the six-foot measuring rod intercepts the shrub cover (Figure 6). As with point intercept, intervals may very. Shrubs are identified by species.

4C-1: 2' interval

4C-2: 5 ' interval

4C-3: 10' interval

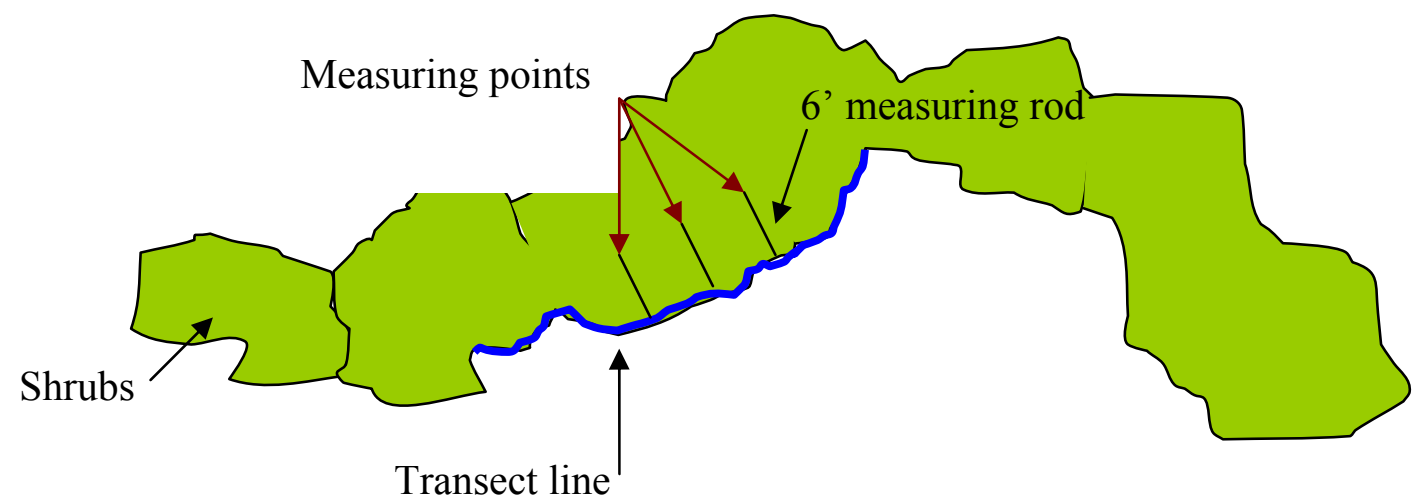

Figure 6. Modified point intercept layout example. 
4D: Complex shrub intercept is used to determine percent shrub cover in multi strata shrub communities. This method is generally associated with point intercept methods whereas overlapping shrubs are identified for each stratum. Percent cover is determined for each of four possible strata as well as total percent shrub cover and overlapping percent cover.

The complex shrub intercept method is identified by adding the suffix "4D" after the appropriate line or point intercept method. For example, "4B-1-4D designates that complex shrub point intercept measurements were taken at two foot intervals. Similarly, 4C-2-4D designates that modified point intercept at five foot intervals was used to determine percent shrub cover for strata in a complex shrub community.

\section{Shrub Height}

5. Shrubs are defined as woody vegetation including trees $<16$ feet in height unless otherwise defined in HEP models. The Regional HEP Team assumes that trees $<16$ feet tall function ecologically more like shrubs than trees.

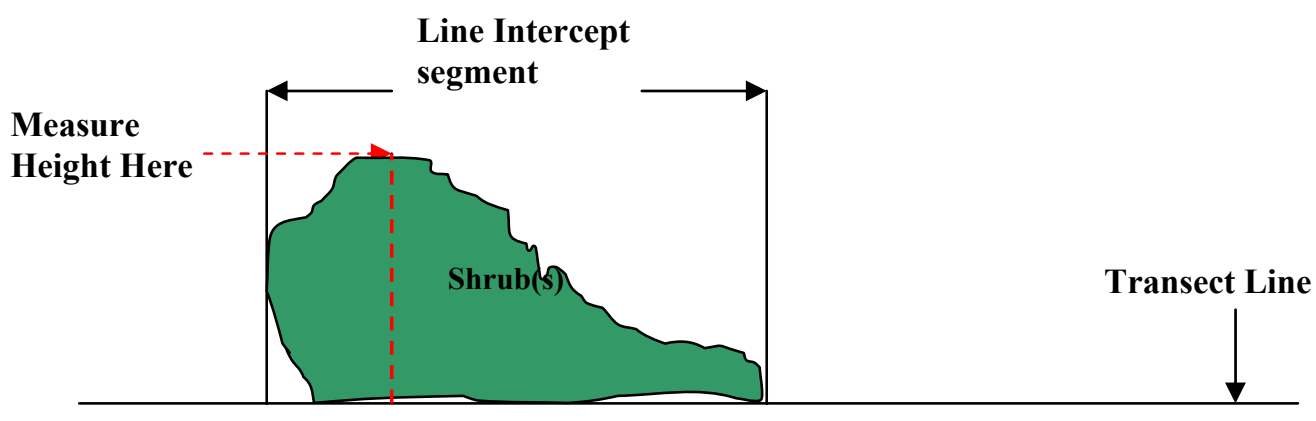

Horizontal View

Figure 7. Line intercept shrub height measurement example.

Shrub height is measured in $10^{\text {ths }}$ of feet at the highest point for each uninterrupted line intercept segment as depicted in Figure 7, or the highest point that crosses each point intercept interval mark on the transect tape (Figure 8).

In structurally complex (overlapping) shrub communities, height is measured for each stratum (maximum of four) as illustrated in Figure 9. It is assumed that shrub height measurements correspond to the method used to determine percent shrub cover. For example, if percent shrub cover is determined using the line intercept 
Eder Acquisition 2007 HEP Report

method (Figure 4), then it is assumed that shrub height will be obtained as illustrated in Figure 7.

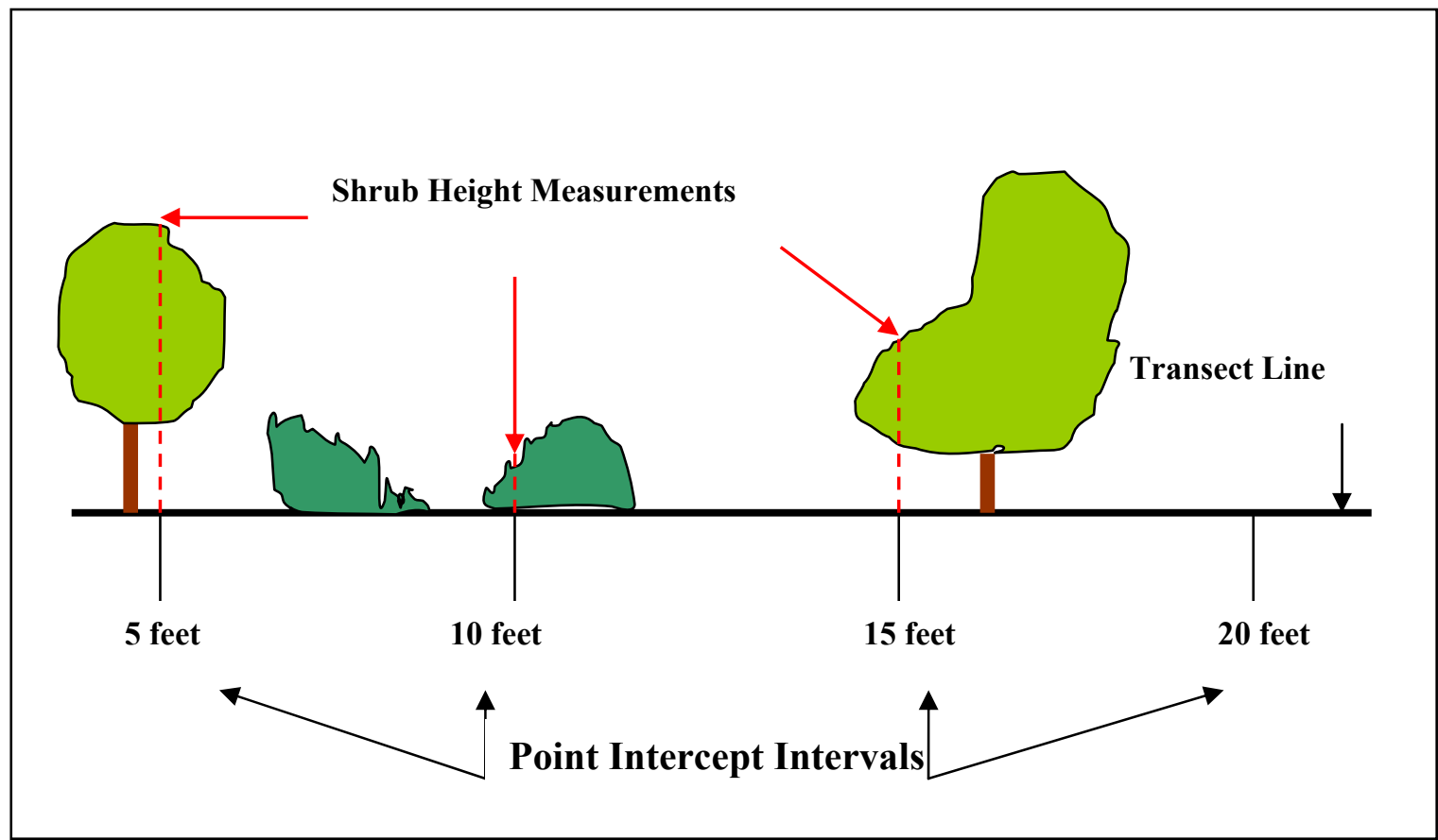

Figure 8. Point intercept shrub height example.

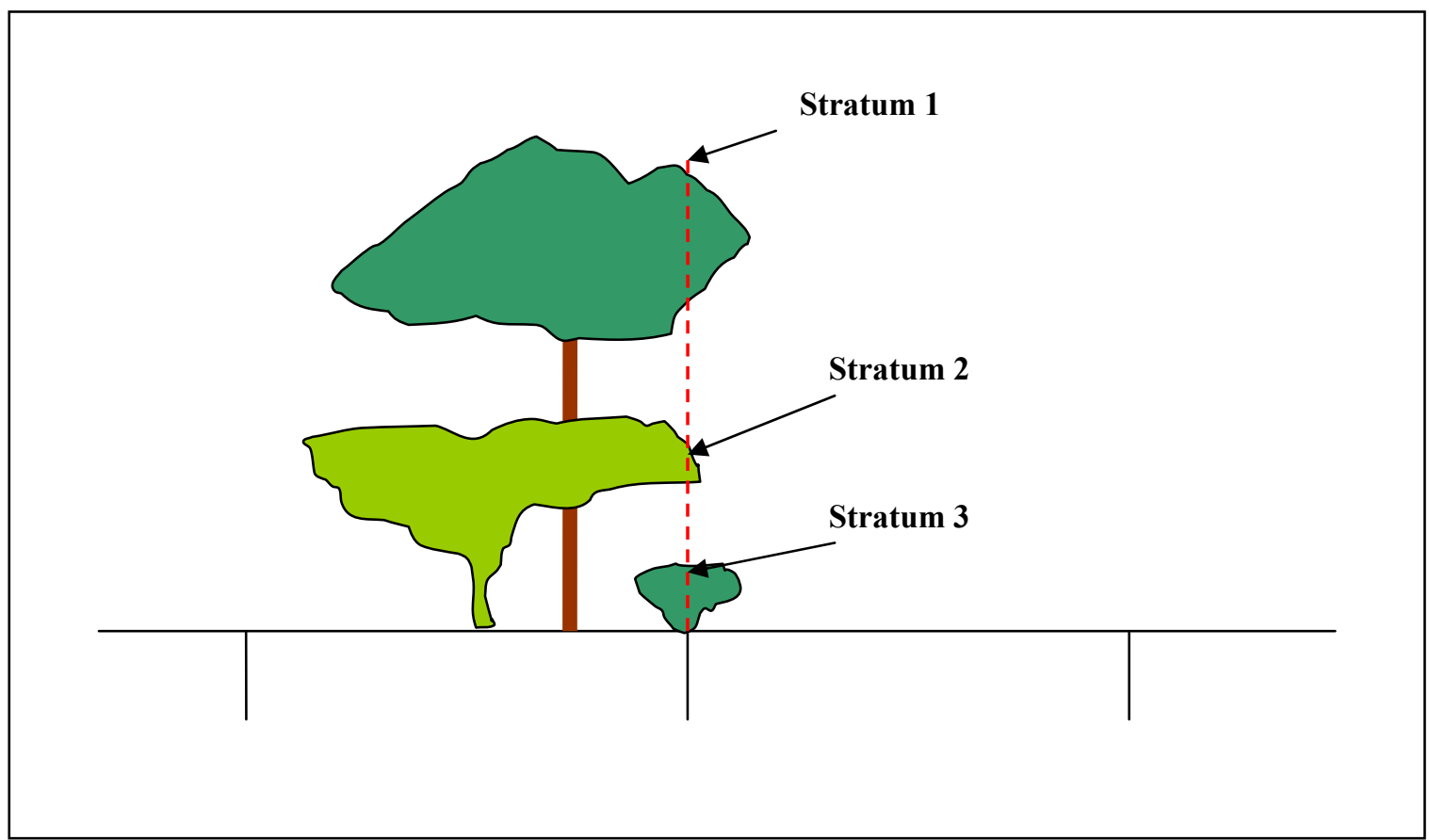

Figure 9. Complex shrub community shrub height measurement example. 
Eder Acquisition 2007 HEP Report

\section{Tree Measurements}

\section{Percent Canopy Cover}

6. Tree canopy cover measurements are recorded at five or ten foot intervals with a densitometer (point intercept). Measurement intervals are determined by visually estimating tree canopy closure prior to initiating the survey. If estimated canopy closure is $<20 \%$ and estimated transect length $\leq 900$ feet, measurements are recorded at five-foot intervals; if estimated canopy closure is $>20 \%$ and estimated transect length is $\geq 600$ feet, ten-foot intervals are used. The size of the sample area strongly influences transect length. In small areas, data from several short (300 foot) transects may be "pooled" in order to determine percent tree canopy cover. As with shrubs, sampled trees are identified by species and the sampling unit is a 100 foot segment of the transect.

6A: 5 ' interval

6B: 10' interval

\section{Height}

7. Tree height is determined generally using a clinometer. In open areas, an electronic height measurement instrument may be used. Measurements are taken at the beginning and end of each transect and at 100 foot intervals. Additional samples may be taken if needed. HEP model variable requirements determine the extent of tree height measurements e.g., multi-canopy, overstory, etc.

Basal Area

8. Tree basal area data is collected at 100-foot intervals using a "factor 10" prism. Each 100-foot interval basal area observation (all tree "hits" at each 100-foot point) is considered an independent sample.

\section{Snag DBH}

9. Snag data is collected on belt transects. RHT members collect snag data in conjunction with tree canopy closure measurements using the same baseline transect. The diameter breast height (DBH) of all snags present within tenth-acre belt transects paralleling the baseline transect is measured. Either the actual DBH is recorded, or snag data is reported by class e.g., 5 snags $<4$ " DBH, 2 snags $>20$ " DBH etc.

Belt transects are 44 feet wide by 100 feet long i.e., 22 feet on each side of the baseline transect. Belt transect layout is depicted in Figure 10. As with shrubs and trees, the sampling unit is each 100-foot segment. 


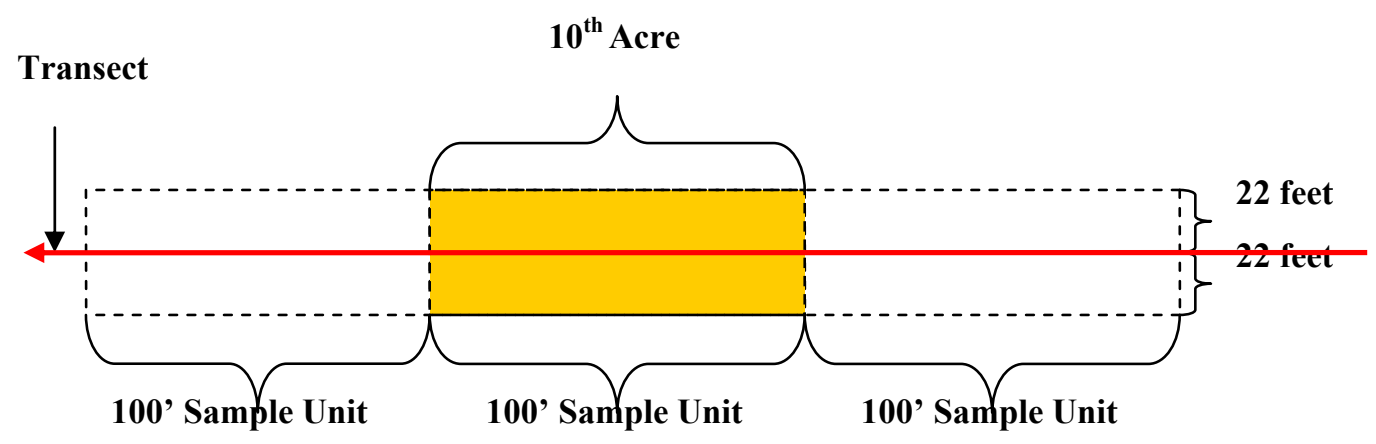

Figure 10. Belt transect layout diagram.

\section{Sample Size Determination}

The process for determining sample size (transect length) varies based on the variable measured. Shrub and tree cover and grid sample sizes are estimated as follows:

The amount of cover within each 100 foot sample unit is divided by sample unit length to obtain percent shrub/tree cover per sample unit (e.g. 10 feet of cover/100 feet $=10 \%$ shrub cover). The standard deviation for each transect is calculated for percent cover data from transect sample units. Sample size (transect length) is then determined through use of the following equation (Avery 1994):

$$
\mathrm{n}=\frac{\mathrm{t}^{2} \mathrm{~s}^{2}}{\mathrm{E}^{2}}
$$

Where: $\mathrm{t}=\mathrm{t}$ value at the 95 percent $(0.05)$ confidence interval for the appropriate degrees of freedom $(\mathrm{df}) ; \mathrm{s}=$ standard deviation; and $\mathrm{E}=$ desired level of precision, or bounds ( \pm 10 percent). Confidence intervals may vary from 80 percent $(0.20)$ to 95 percent $(0.05)$ depending on habitat variable heterogeneity and project management needs. The same method is used to determine sample size for micro plot samples based on total percent cover for herbaceous species. 
Eder Acquisition 2007 HEP Report

\section{References}

Avery, T.E., H. E. Burkhart. 1994. Forest measurements. $4^{\text {th }}$ edition. John Wiley and Sons. New York, NY.

BLM. 1998. Measuring and monitoring plant populations. BLM Technical Reference 1730-1. BLM National Business Center. Denver, CO. 477 p.

Block, W.M., W.L. Kendall, M.L. Morrison, and M. Dale Strickland. 2001. Wildlife study design. Springer Press. New York, NY. 210 p.

Hays, R. L., C. Summers, and W. Seitz. 1981. Estimating habitat variables. Western Energy and land Use Team. Fort Collins, CO: U.S. Fish and Wildlife Service.

Robel, R.J., J. N. Dayton, A.D. Hulbert. 1975. Relationship between visual obstruction measurements and weight of grassland vegetation. Journal of Range Management. 23: 295. 


\section{Appendix C - Transect Start Point Locations}

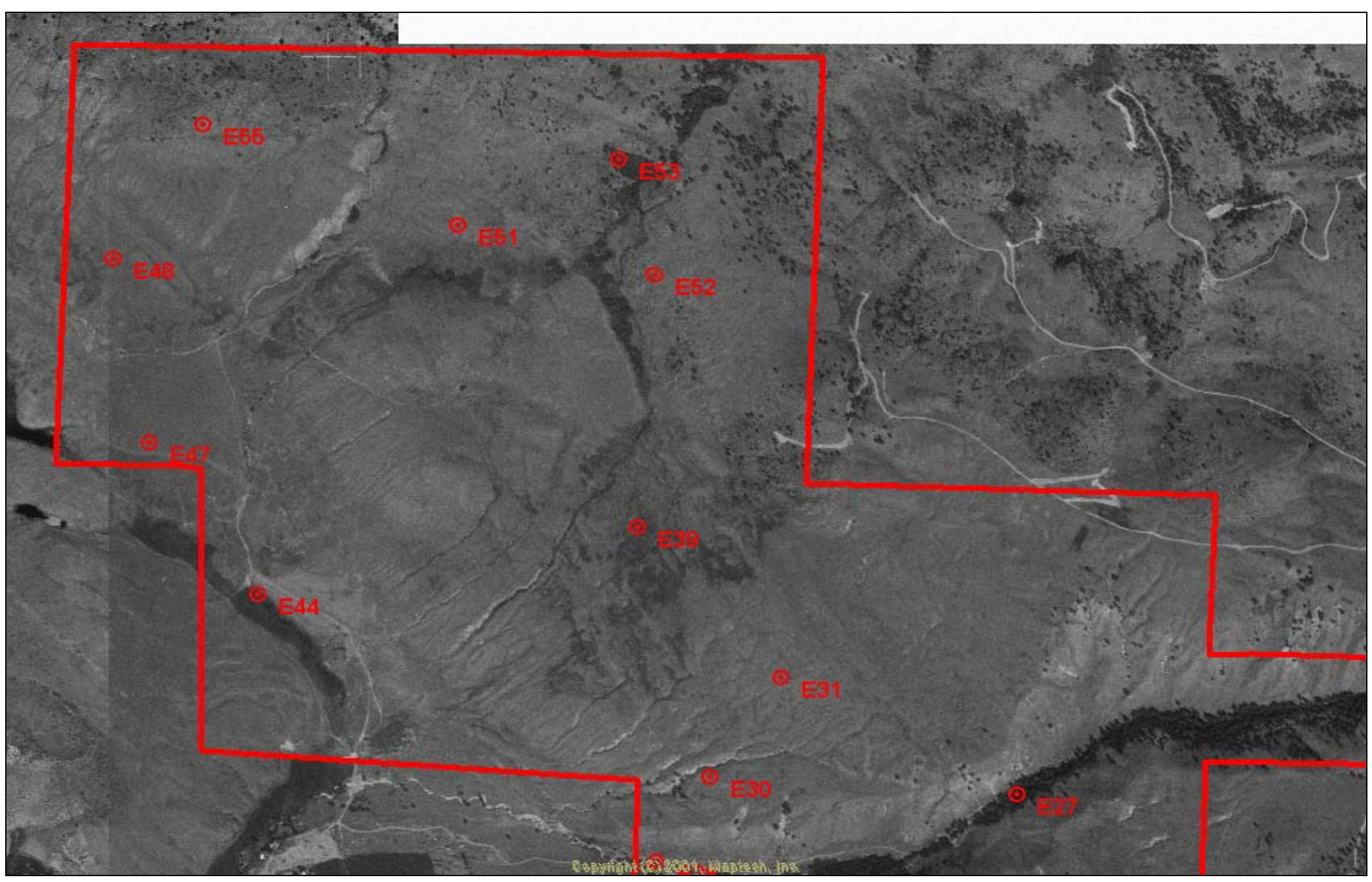




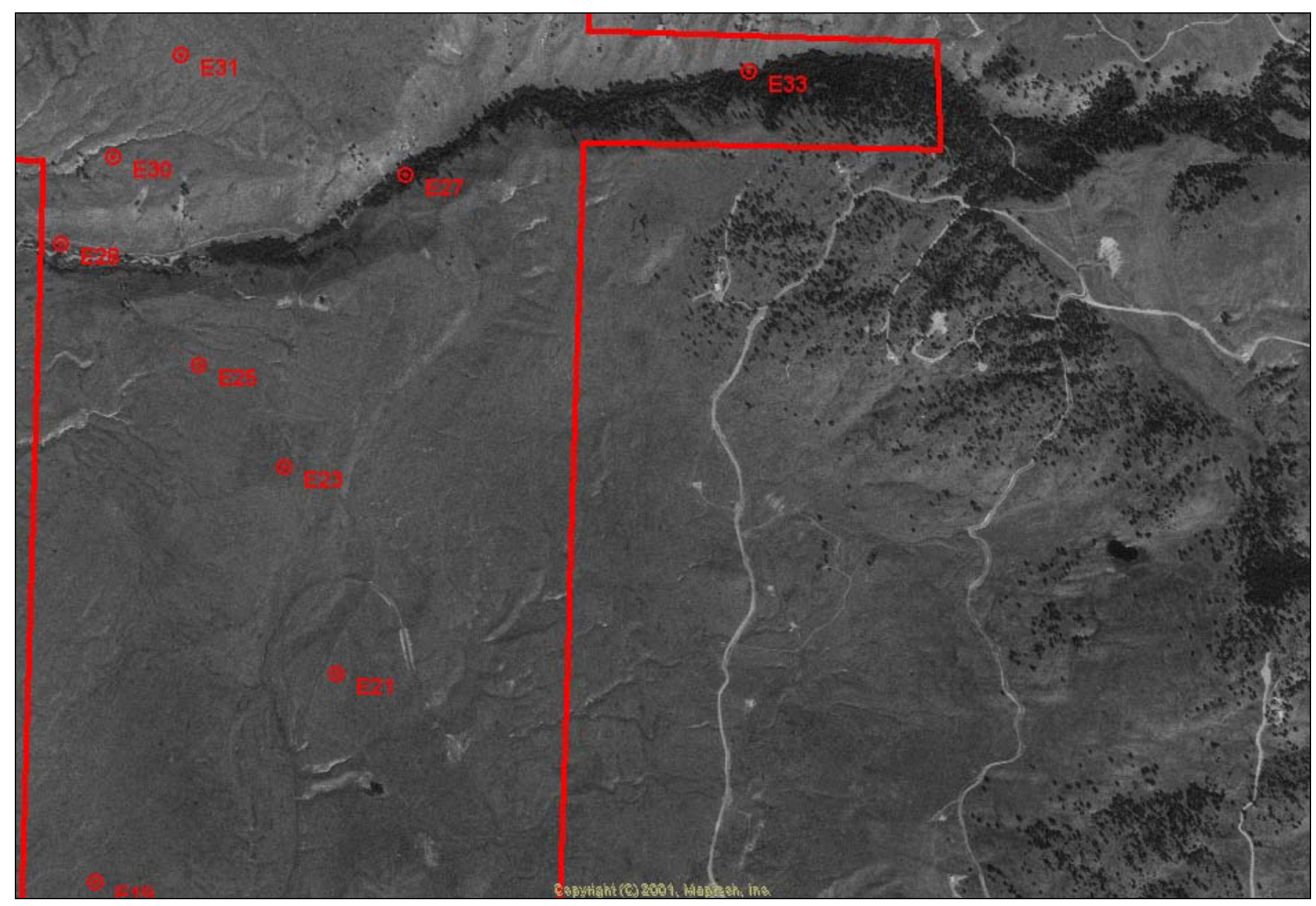




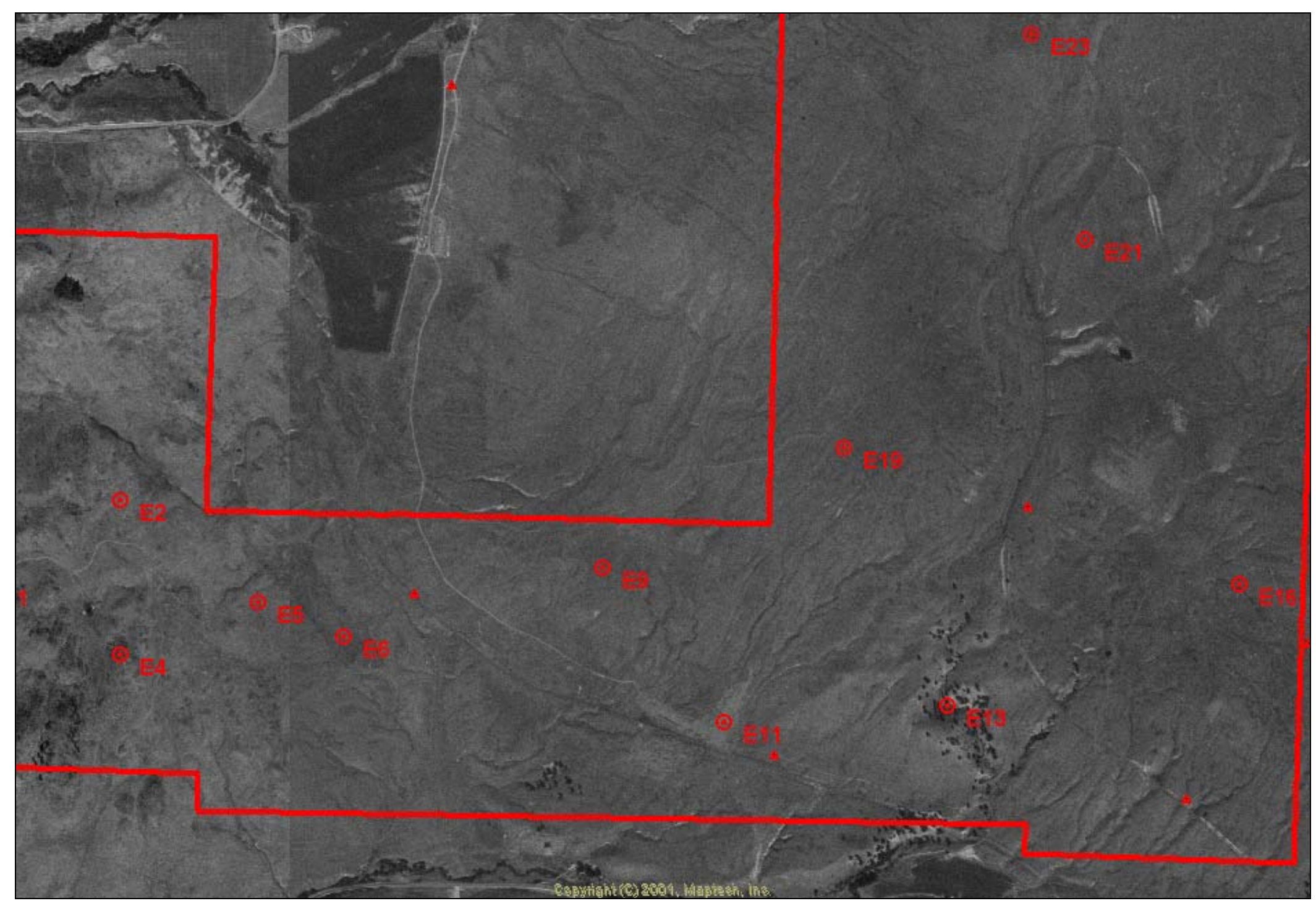




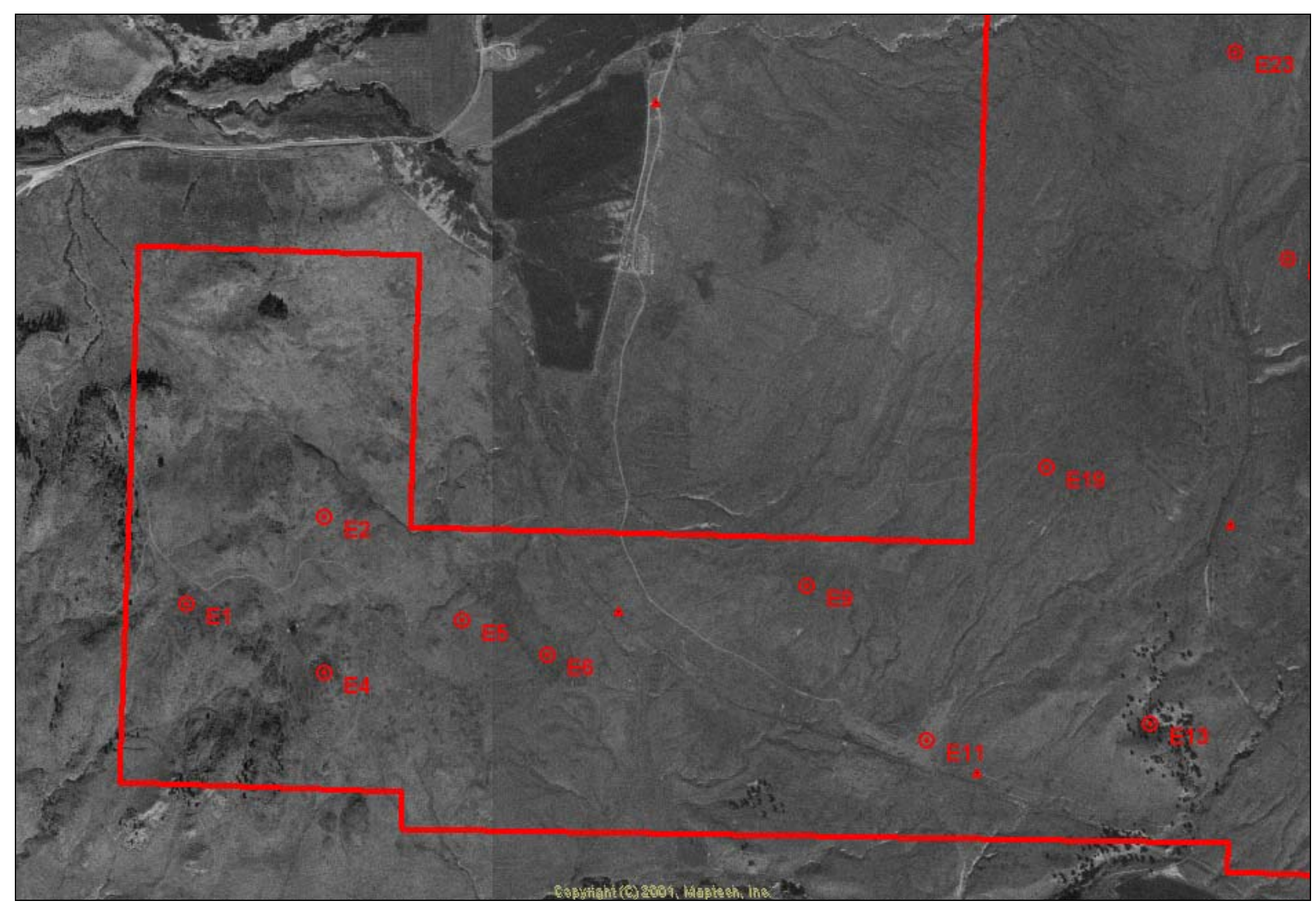


Eder Acquisition 2007 HEP Report

\section{Appendix D - Transect Photographs}

Transect 1

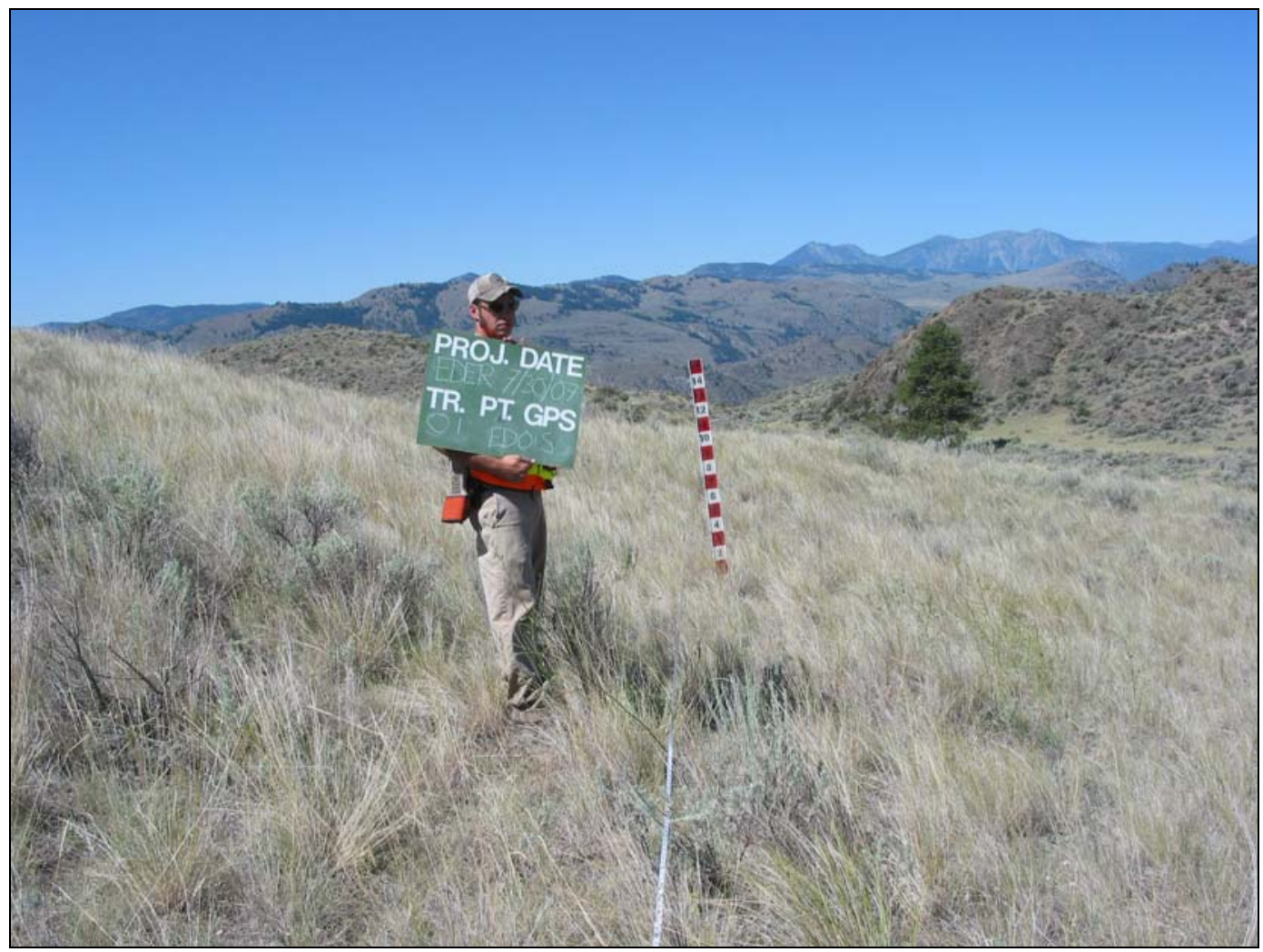


Eder Acquisition 2007 HEP Report

Transect 2

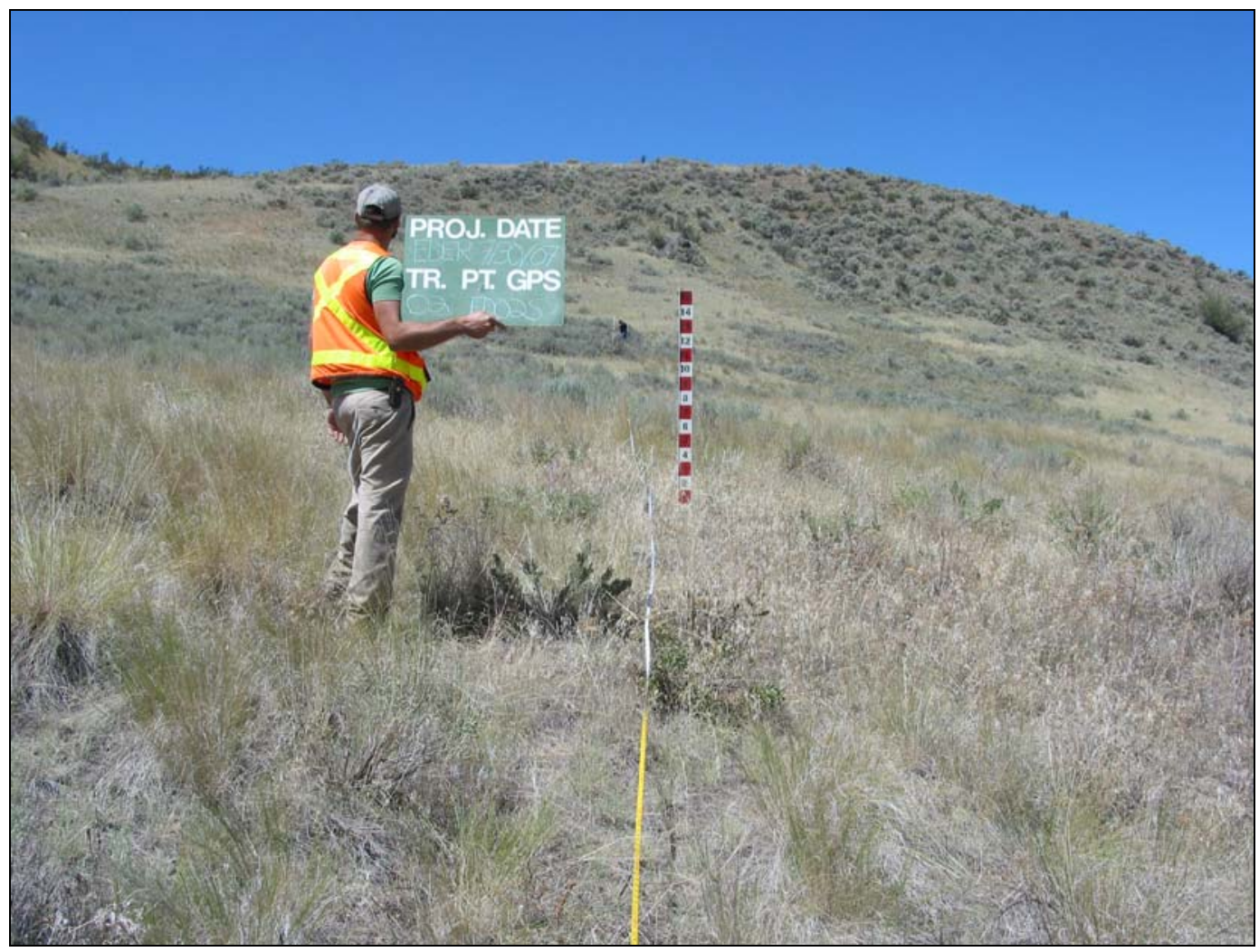

Transect 4

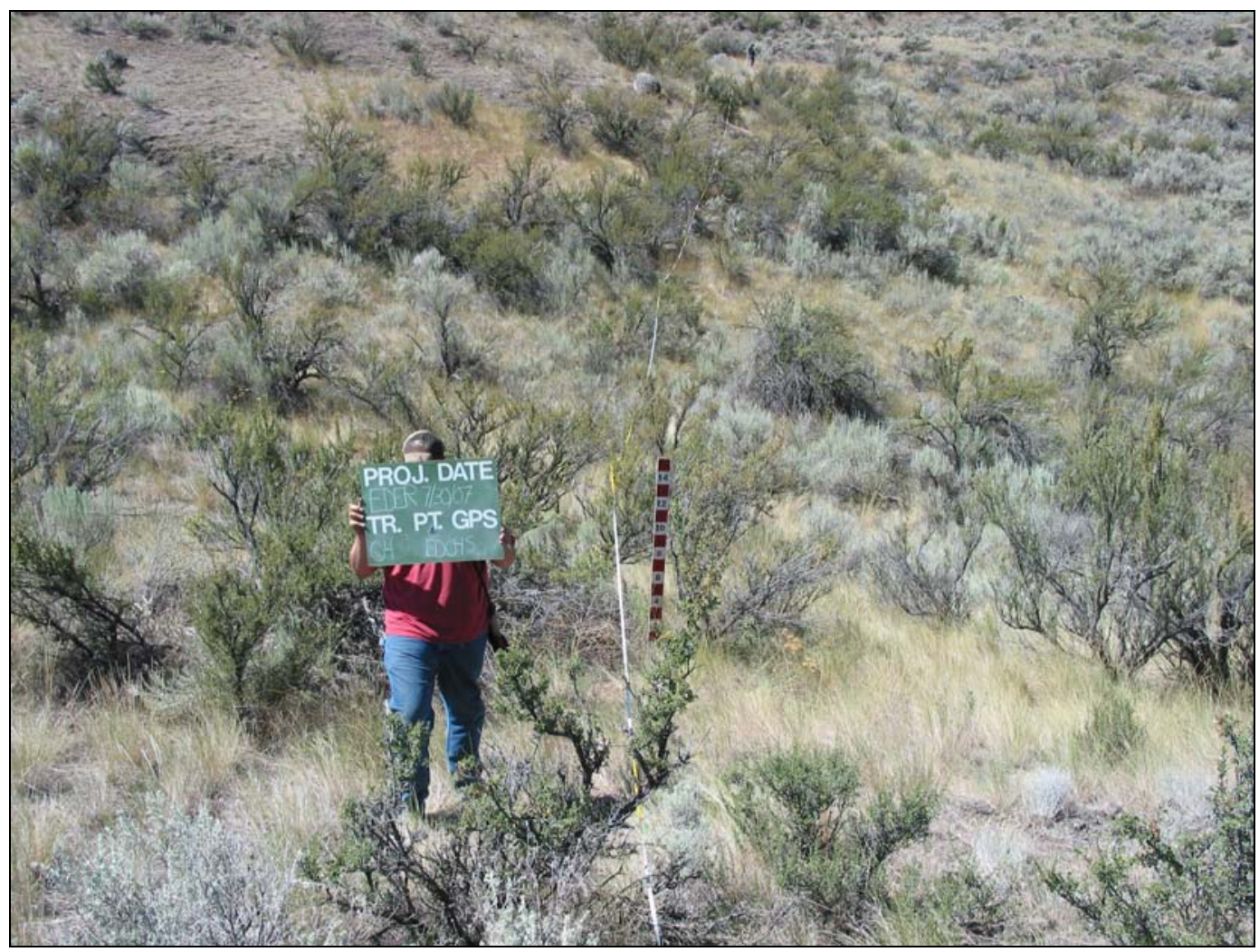


Eder Acquisition 2007 HEP Report

\section{Transect 5}

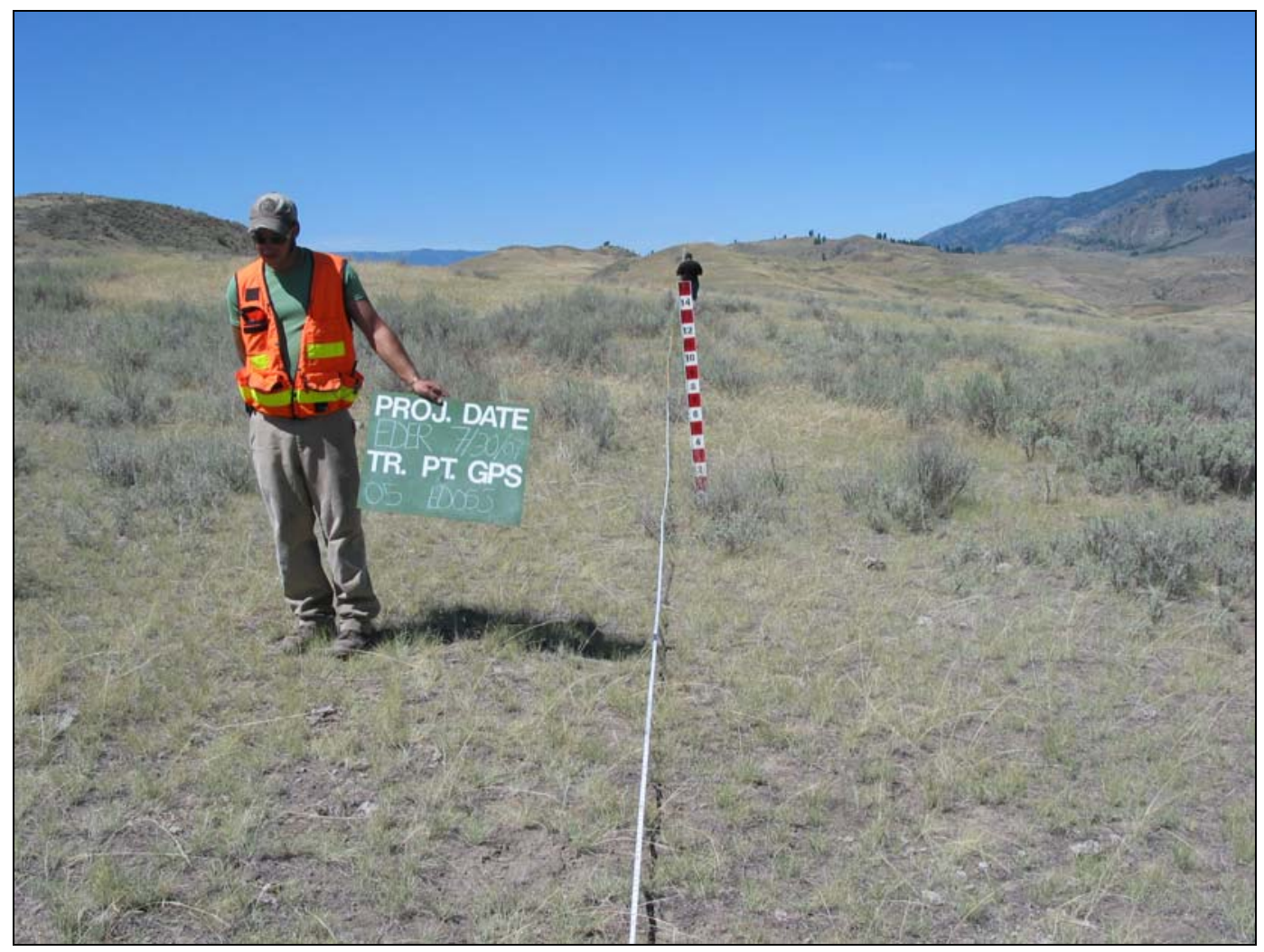

Transect 6

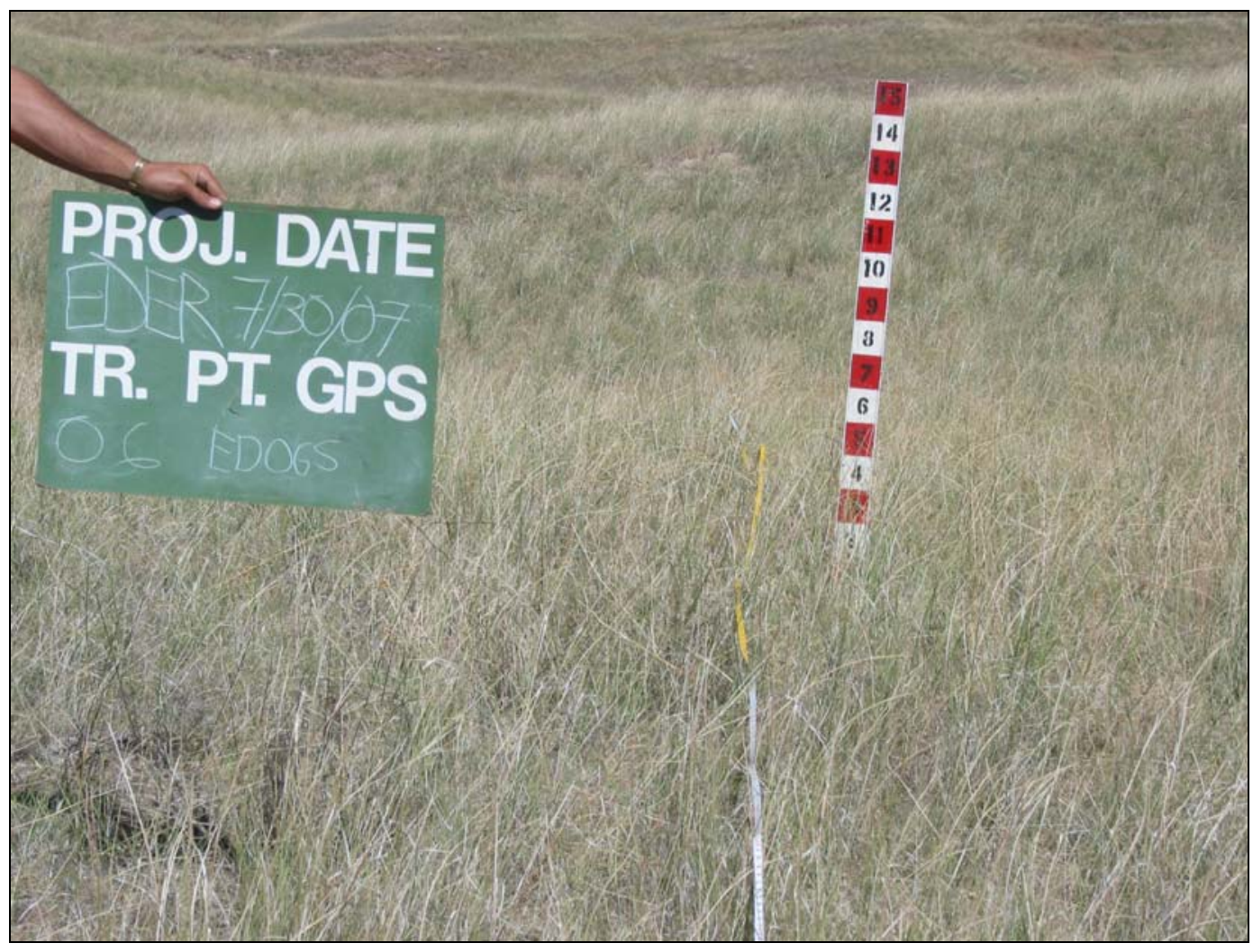


Eder Acquisition 2007 HEP Report

\section{Transect 9}

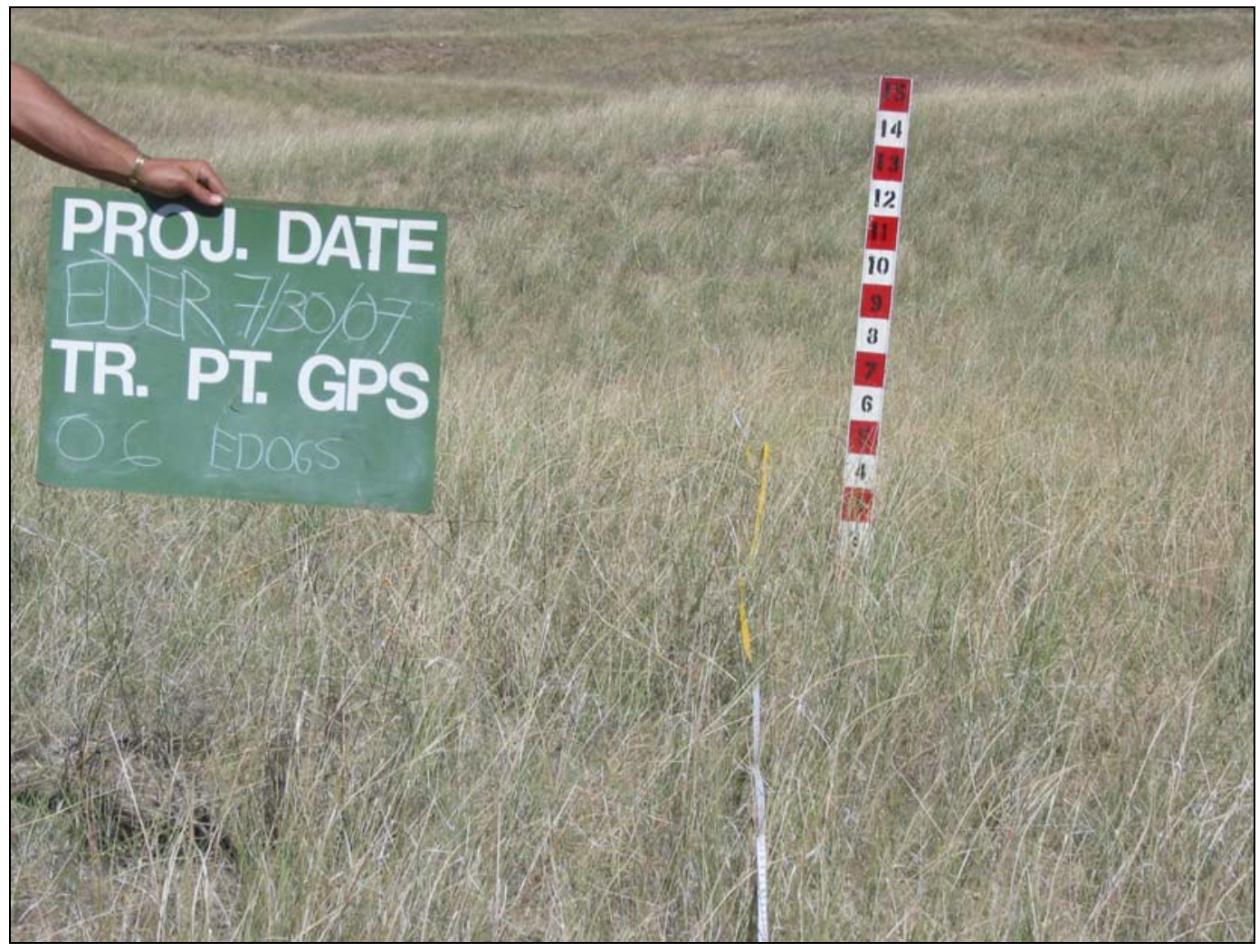

Transect 11

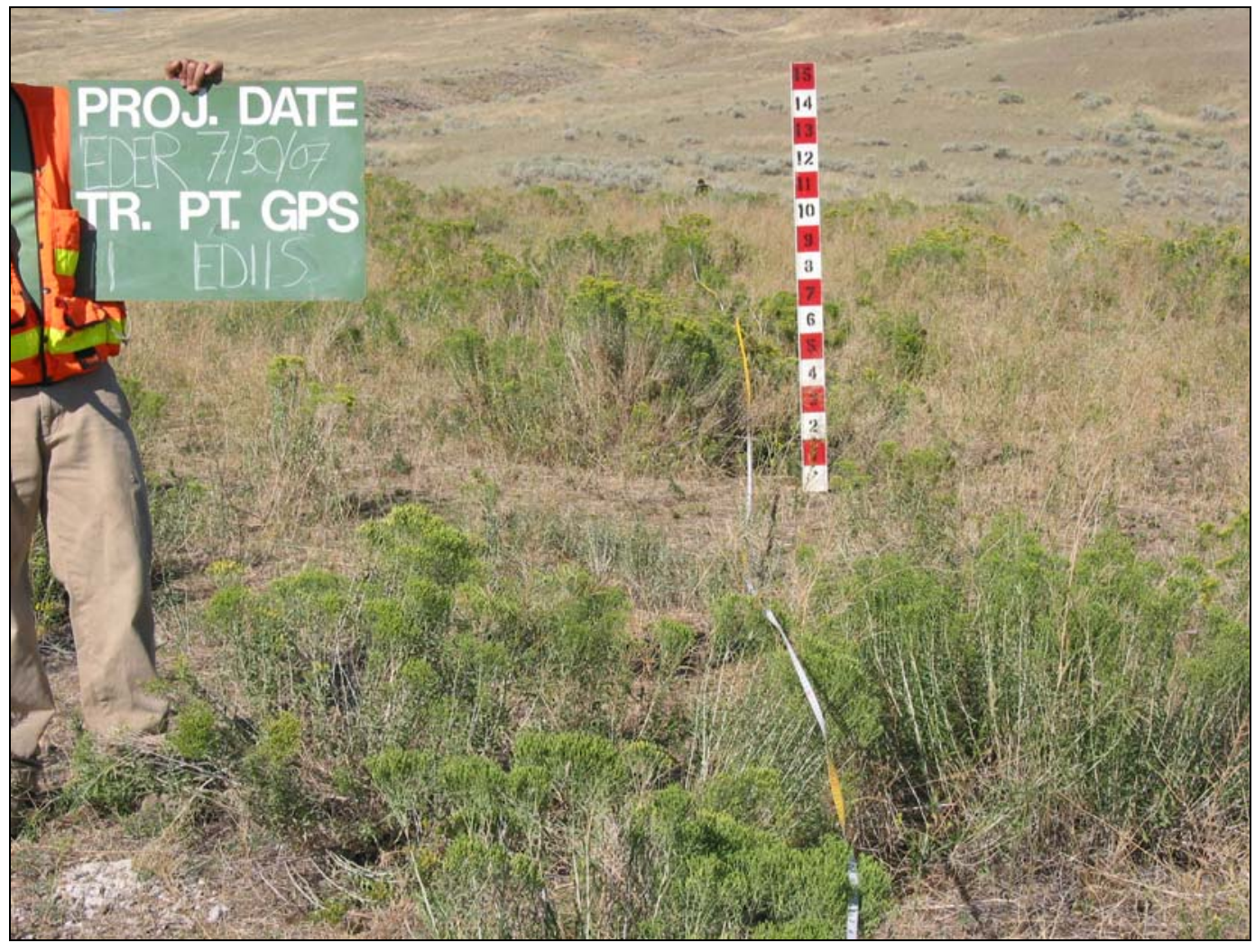


Eder Acquisition 2007 HEP Report

\section{Transect 13}

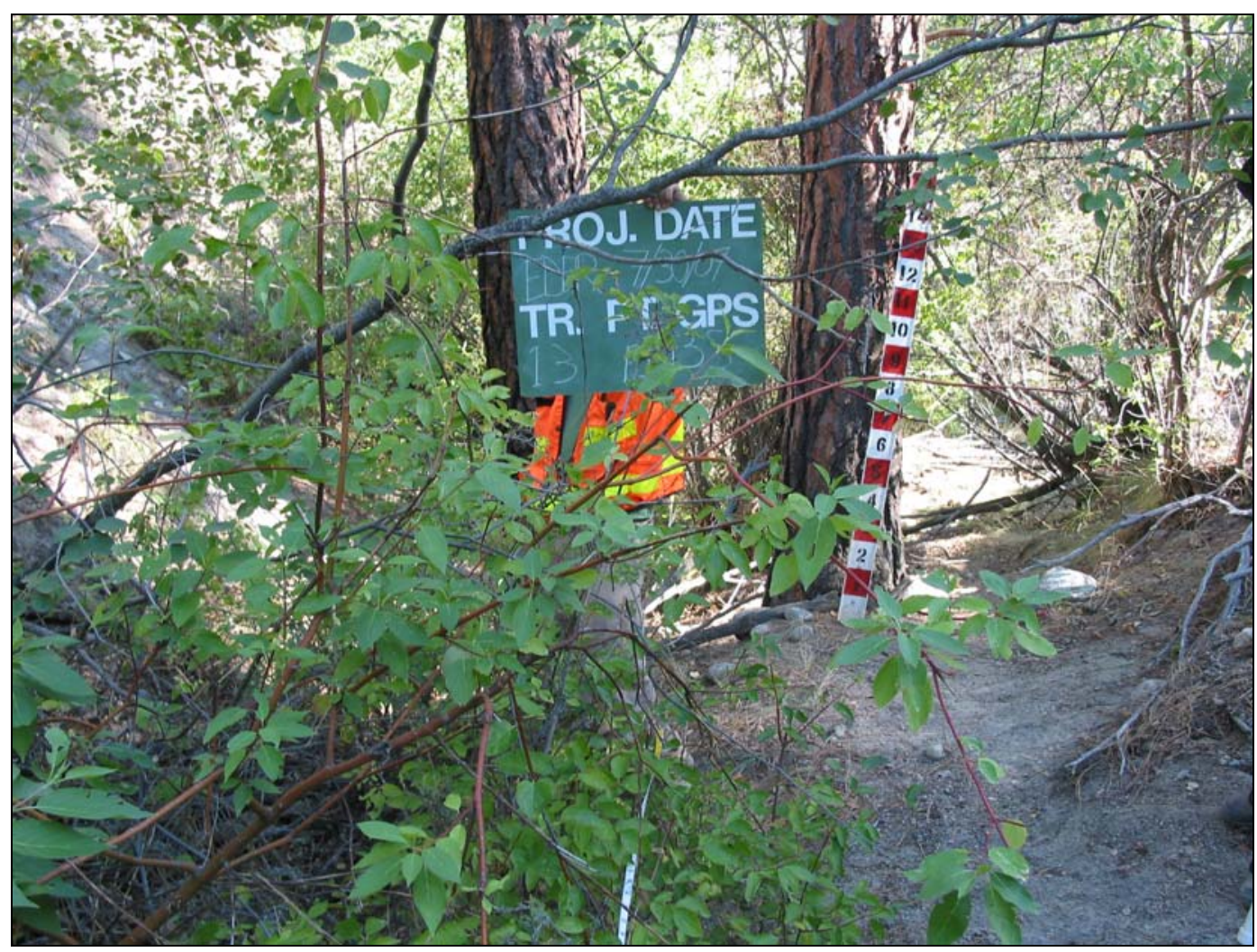

\section{Transect 16}

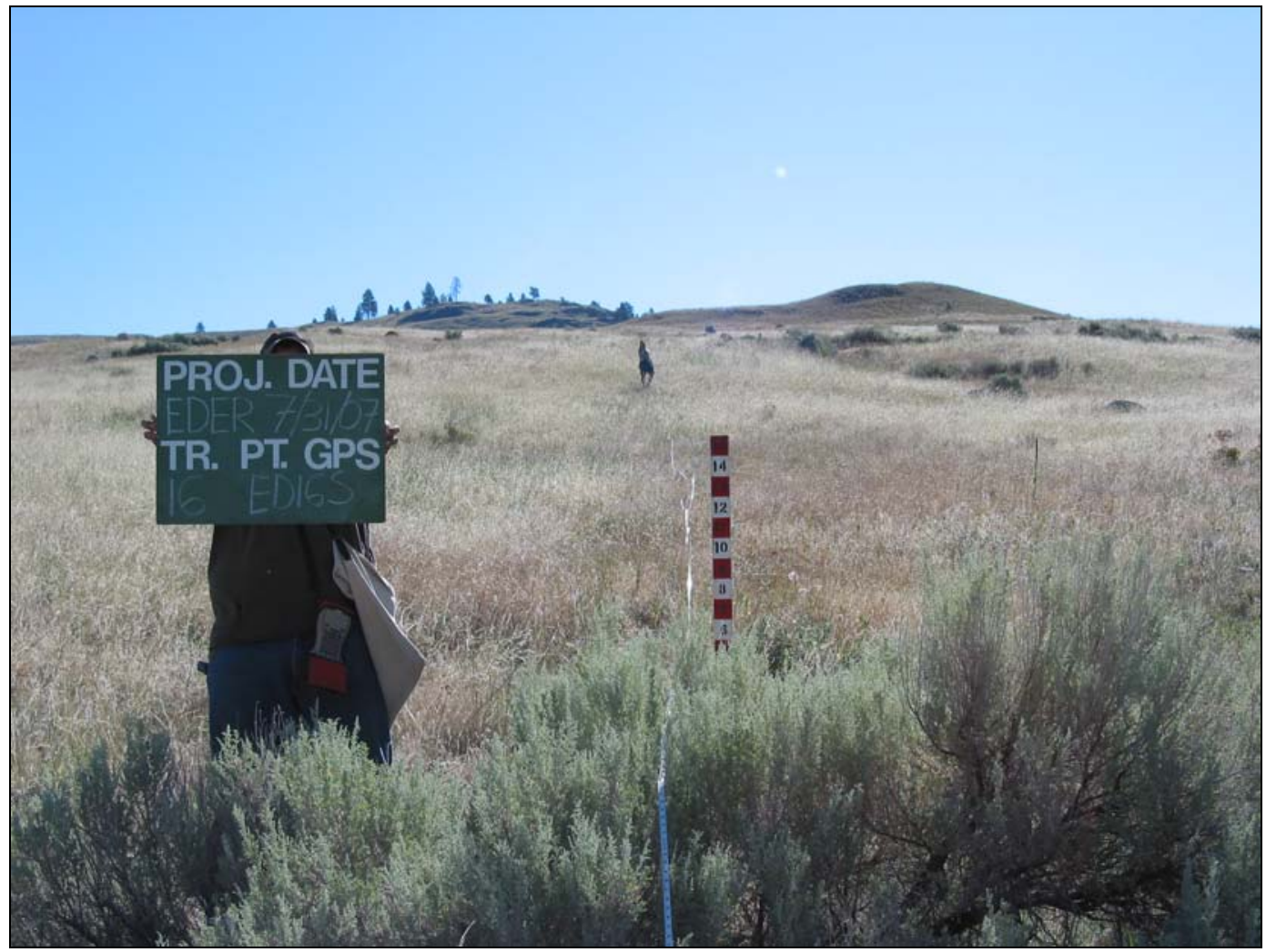


Eder Acquisition 2007 HEP Report

\section{Transect 19}

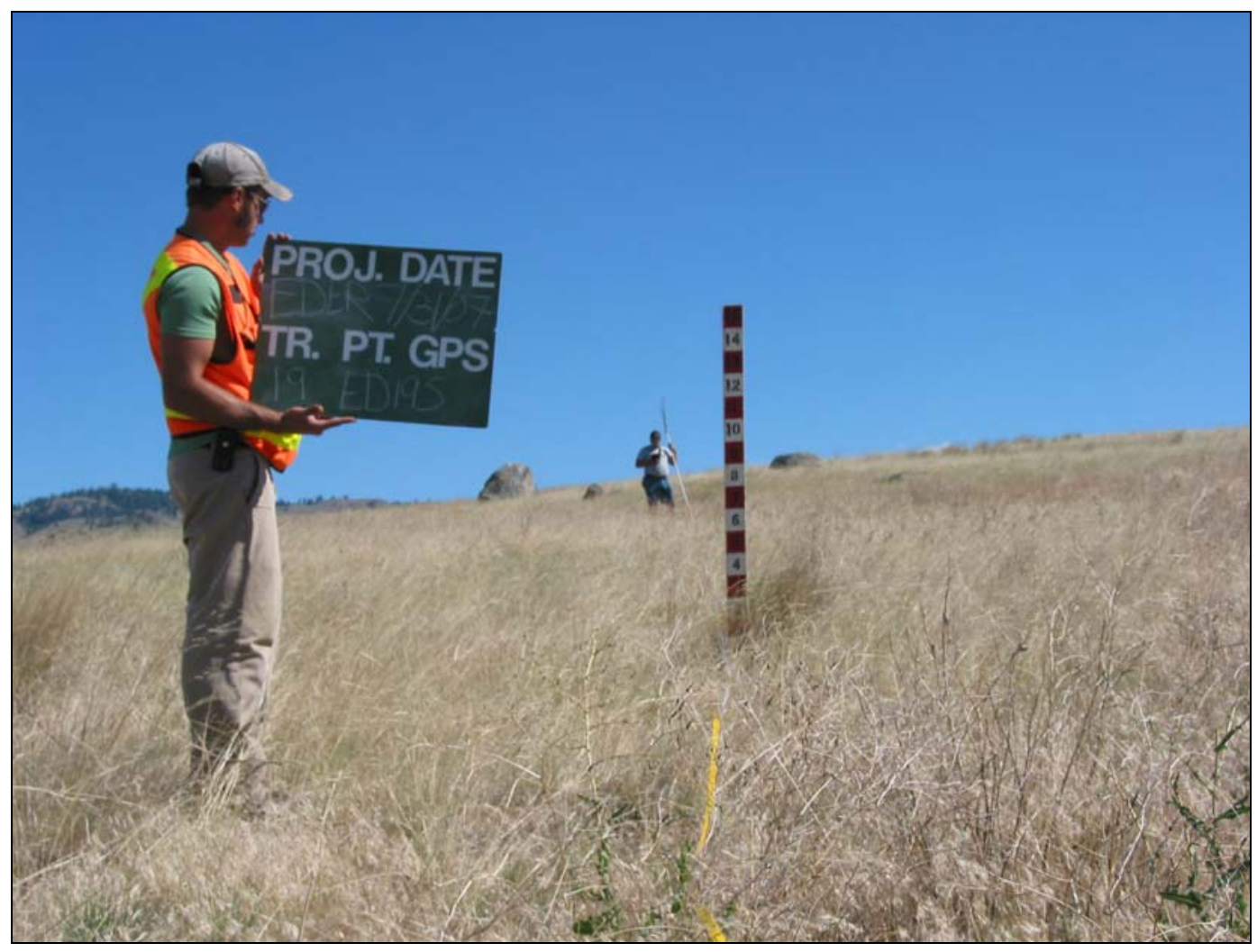

Transect 21

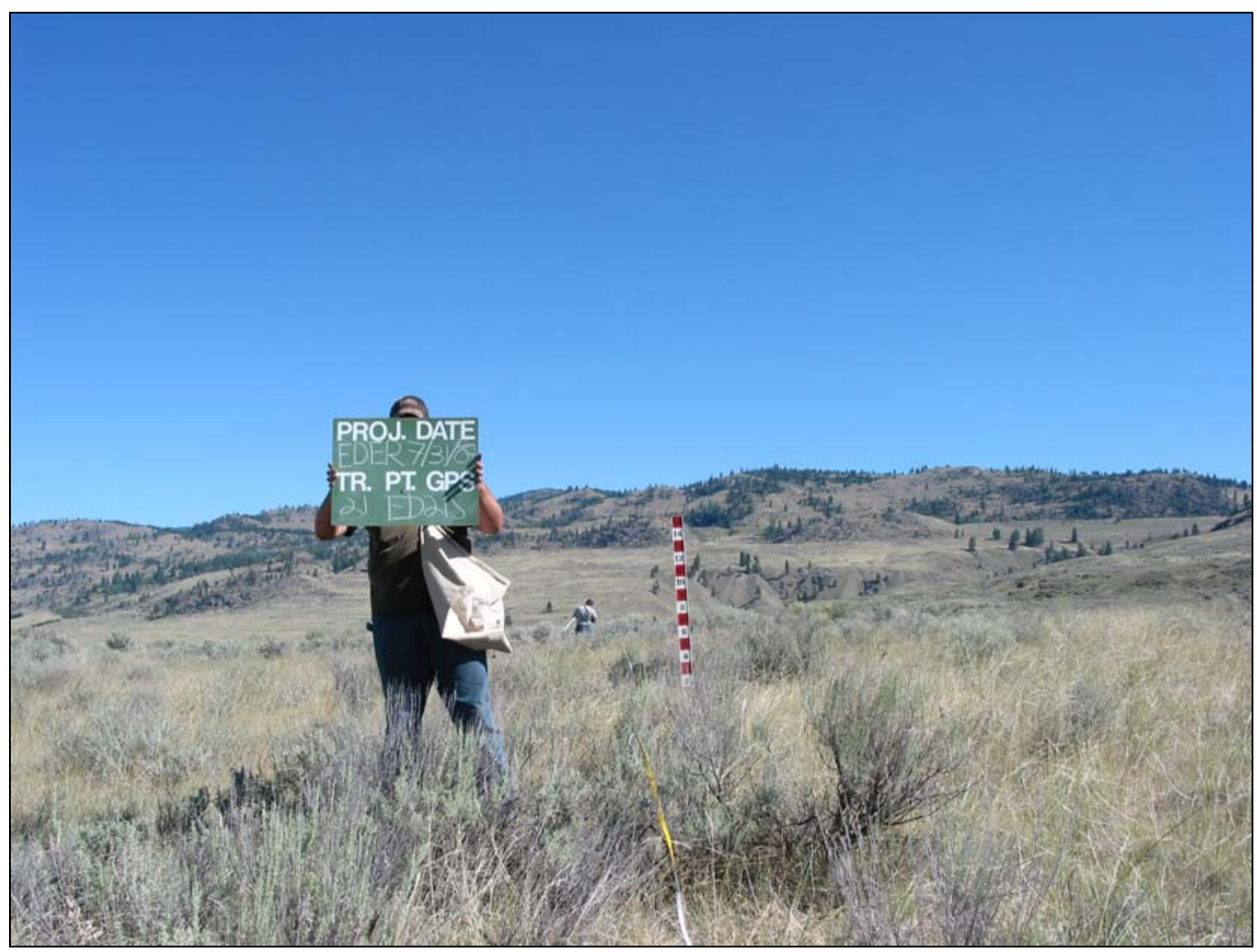


Eder Acquisition 2007 HEP Report

\section{Transect 23}

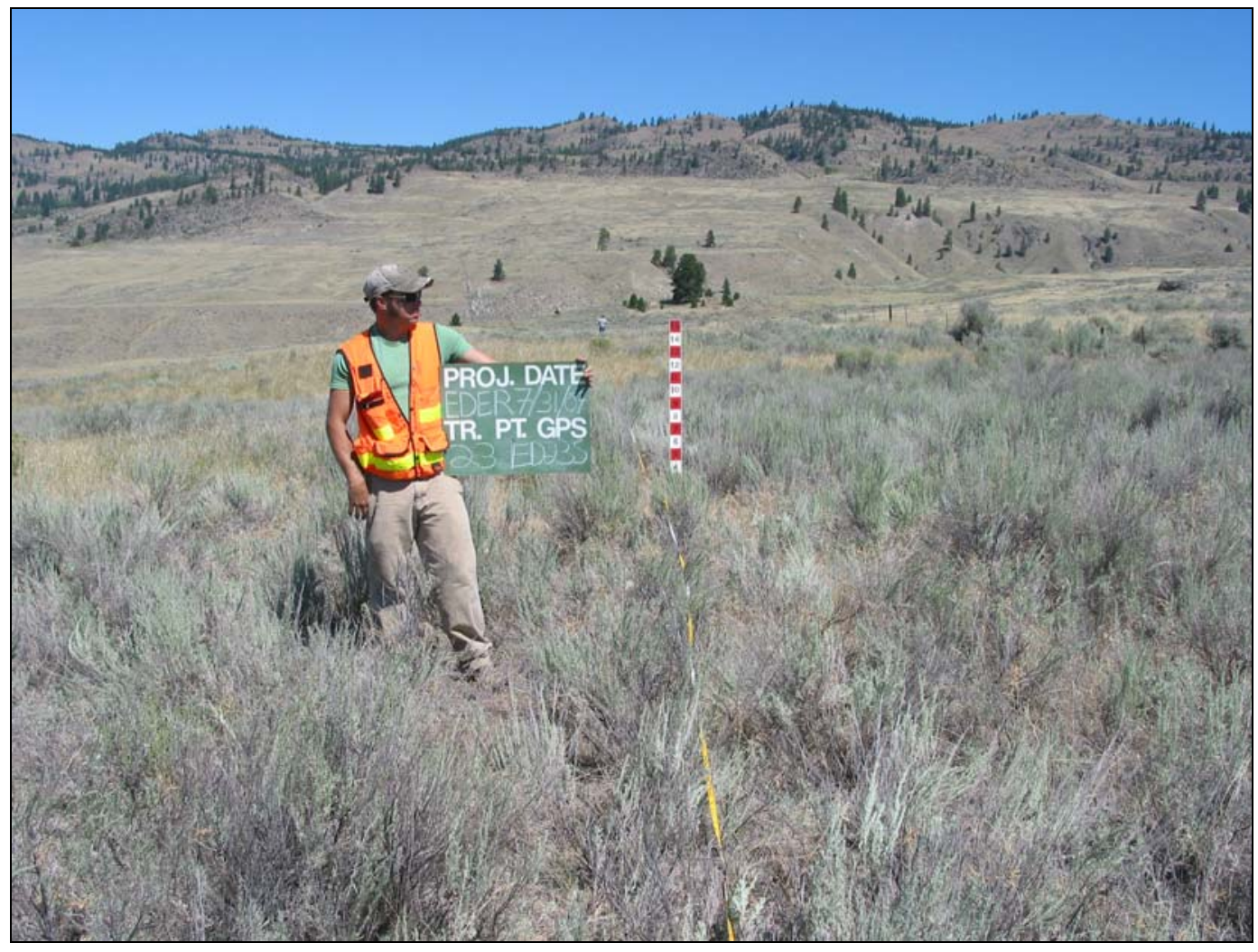

\section{Transect 25}

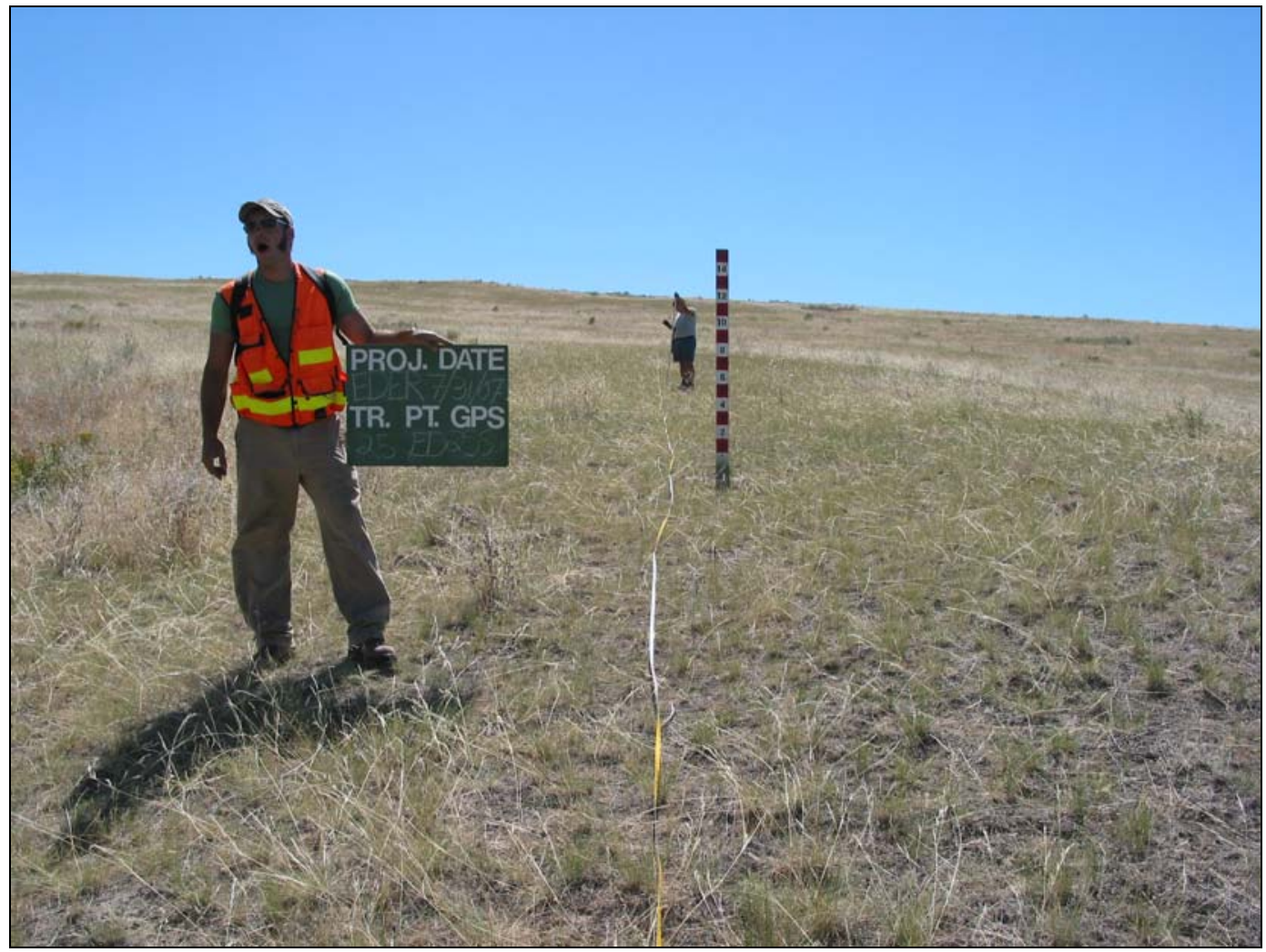


Eder Acquisition 2007 HEP Report

\section{Transect 27}

No Photograph

\section{Transect 28}

No Photograph

\section{Transect 30}

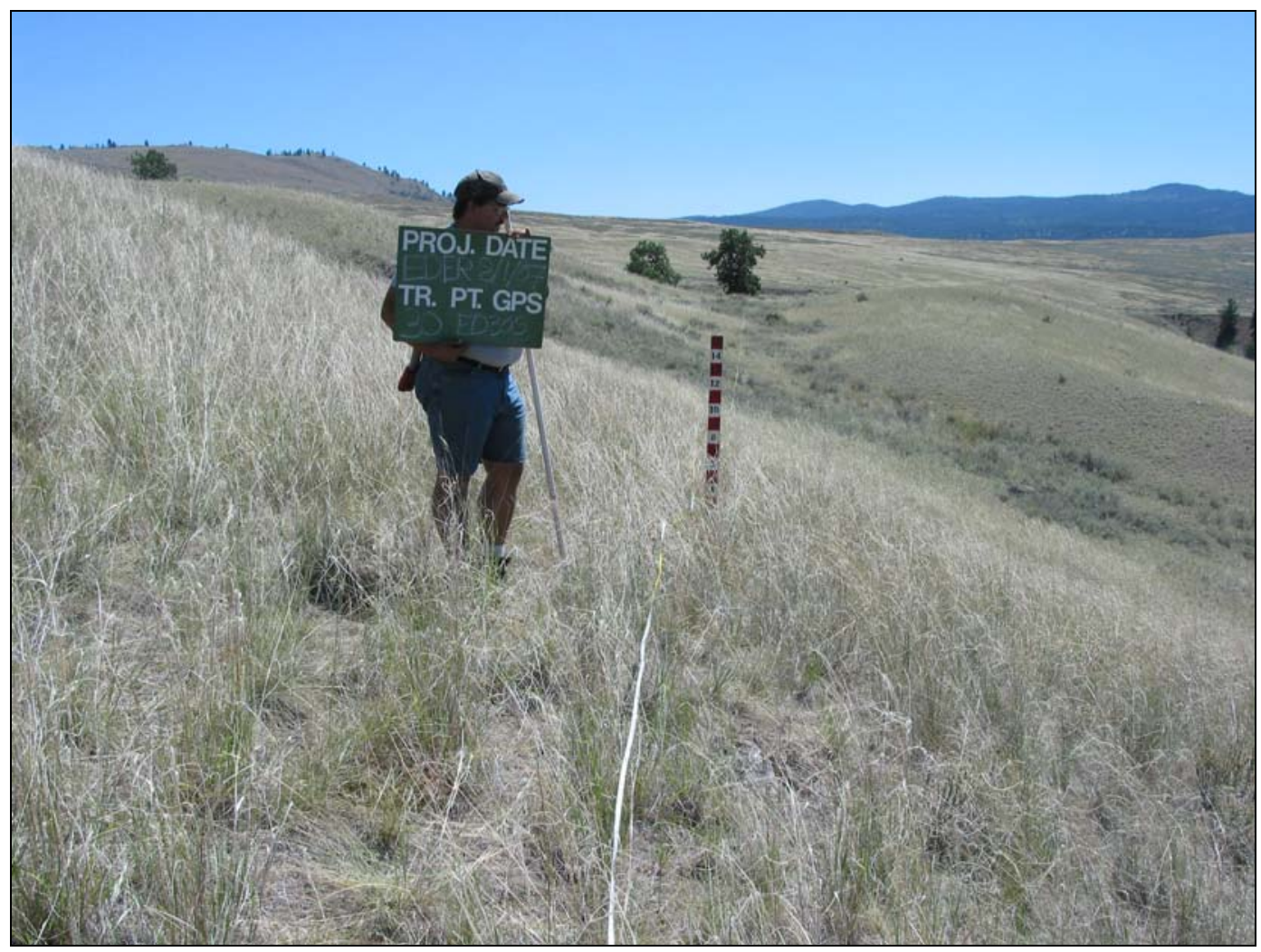


Eder Acquisition 2007 HEP Report

\section{Transect 31}

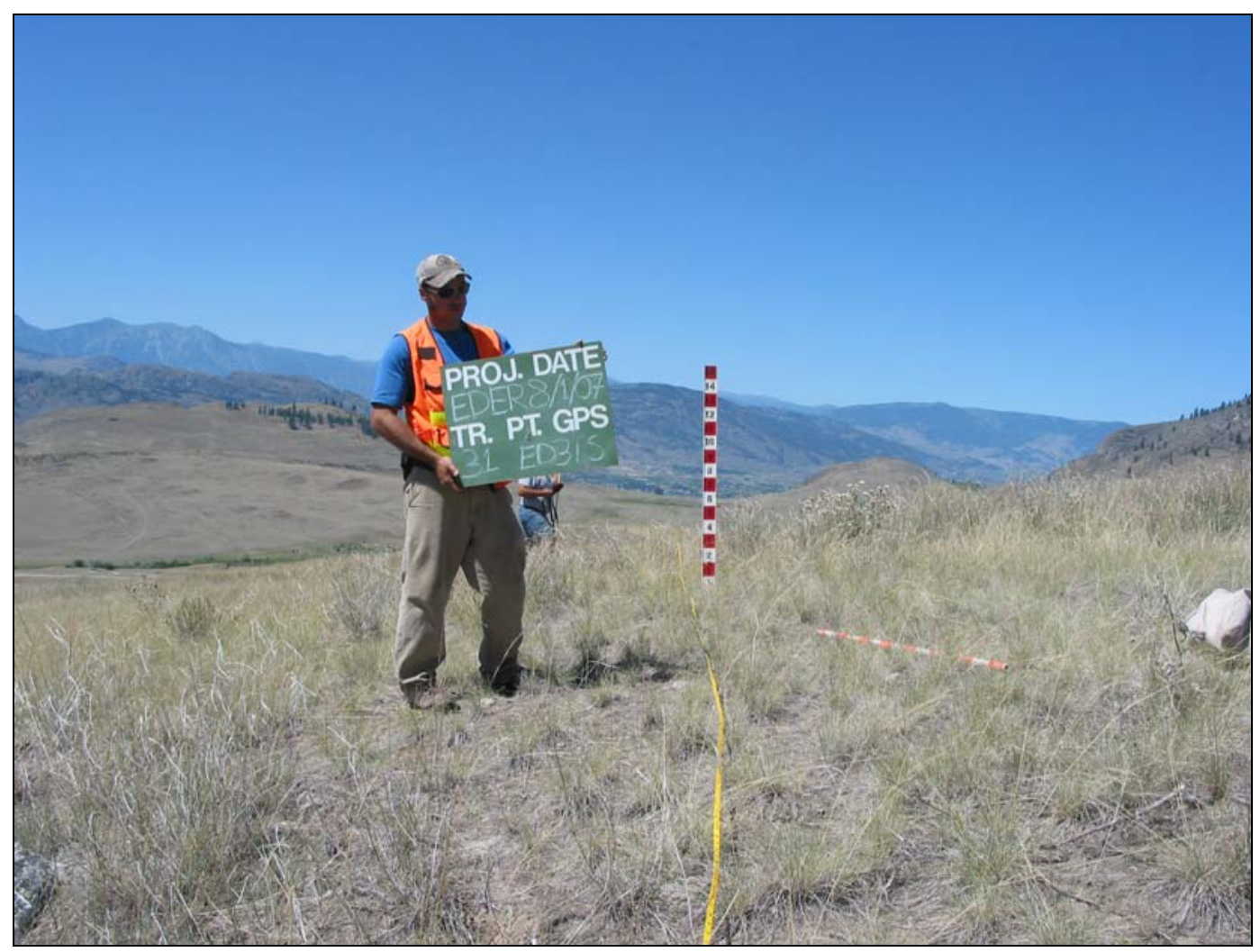

Transect 33

No Photograph 
Eder Acquisition 2007 HEP Report

\section{Transect 39}

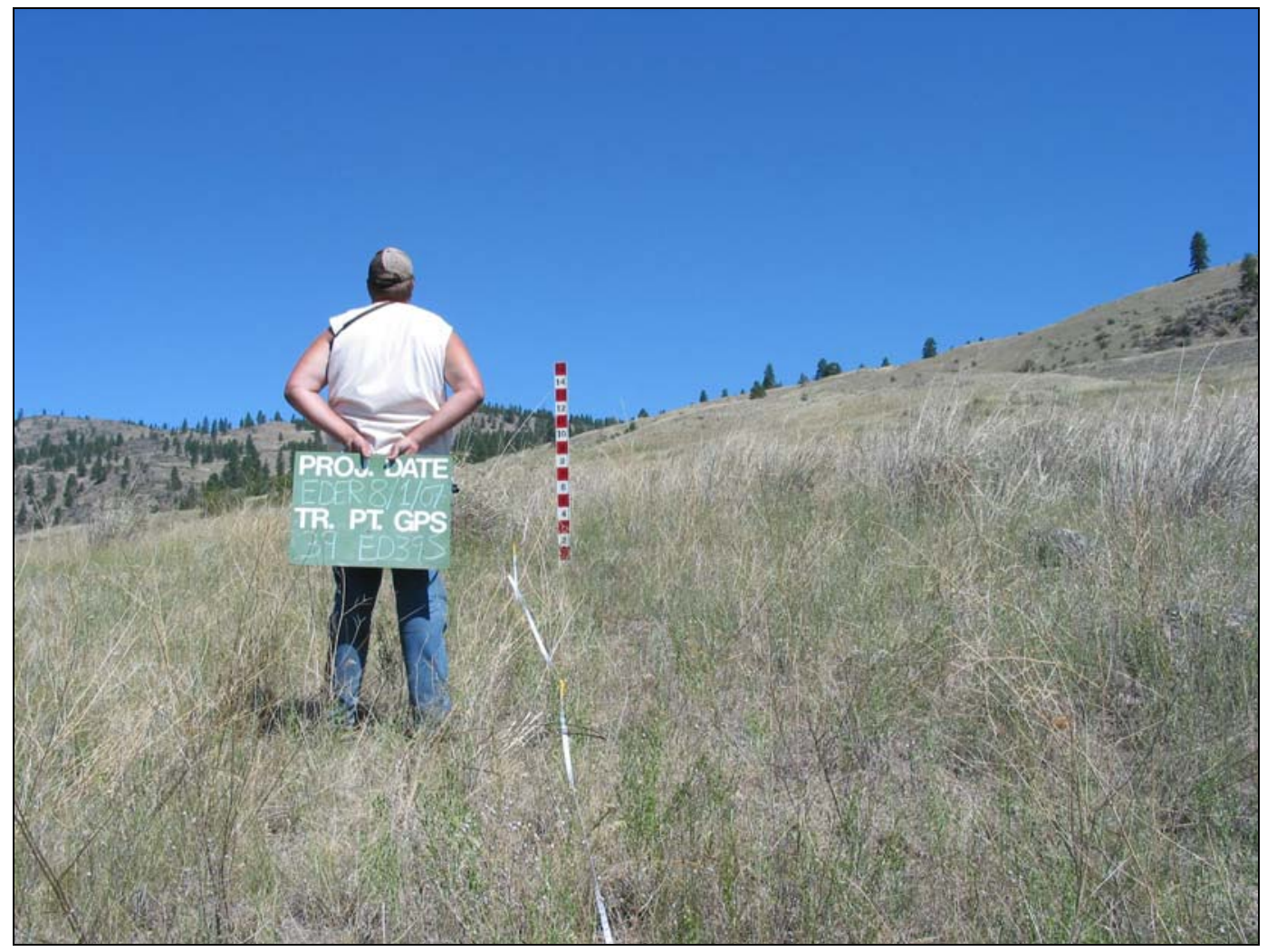

\section{Transect 44}

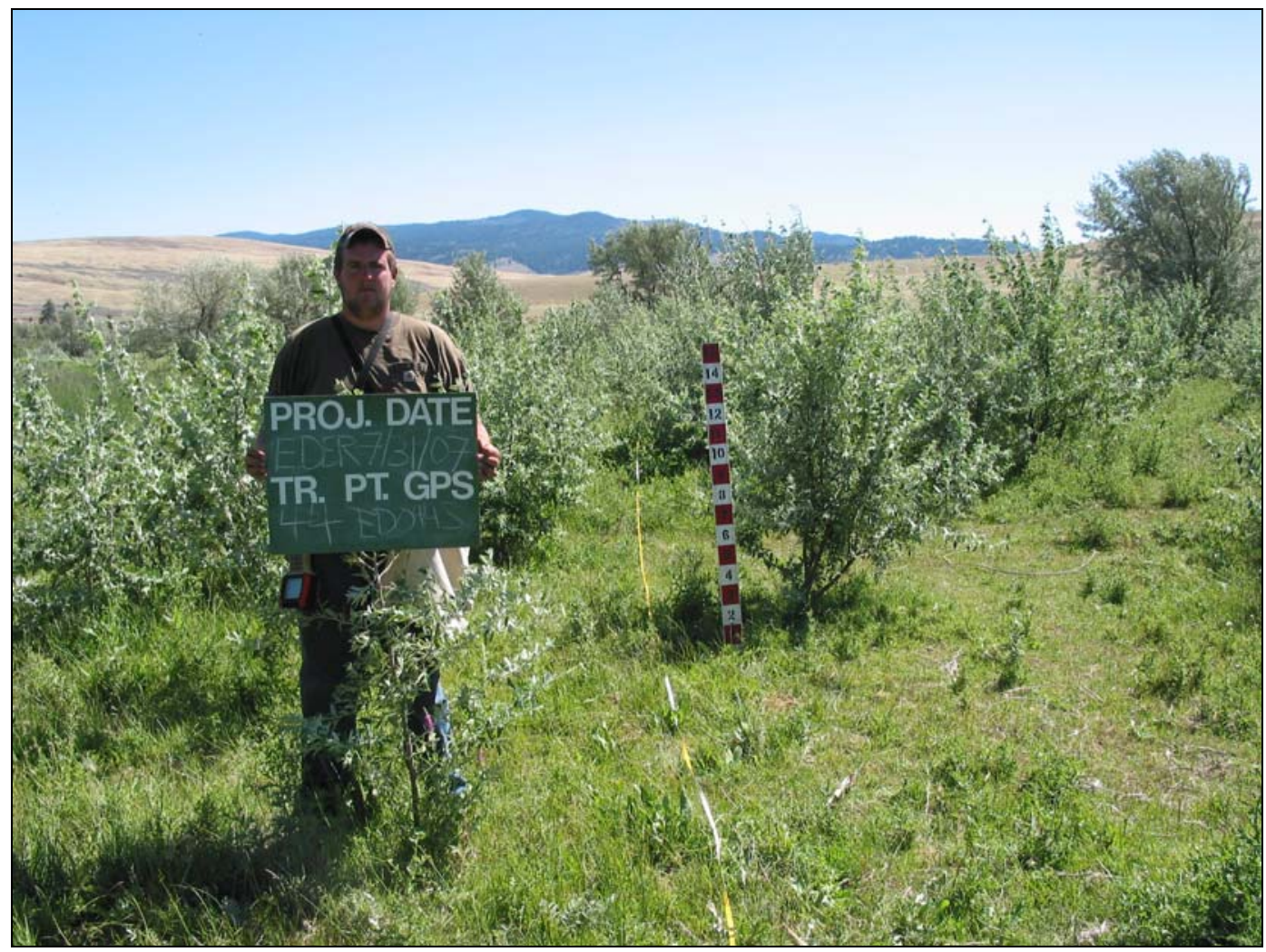


Eder Acquisition 2007 HEP Report

\section{Transect 48}

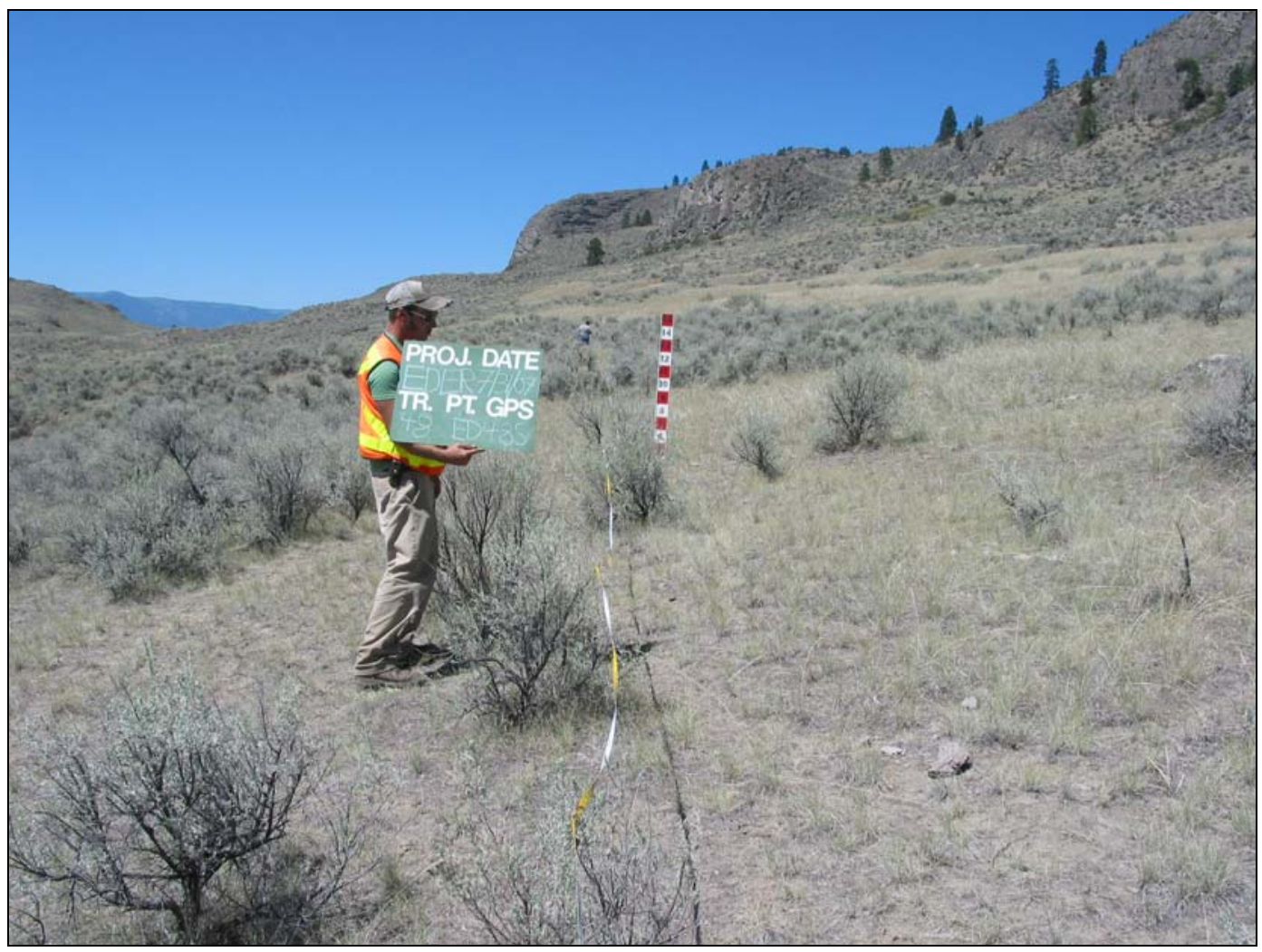

Transect 51

No Photograph 
Eder Acquisition 2007 HEP Report

\section{Transect 53}

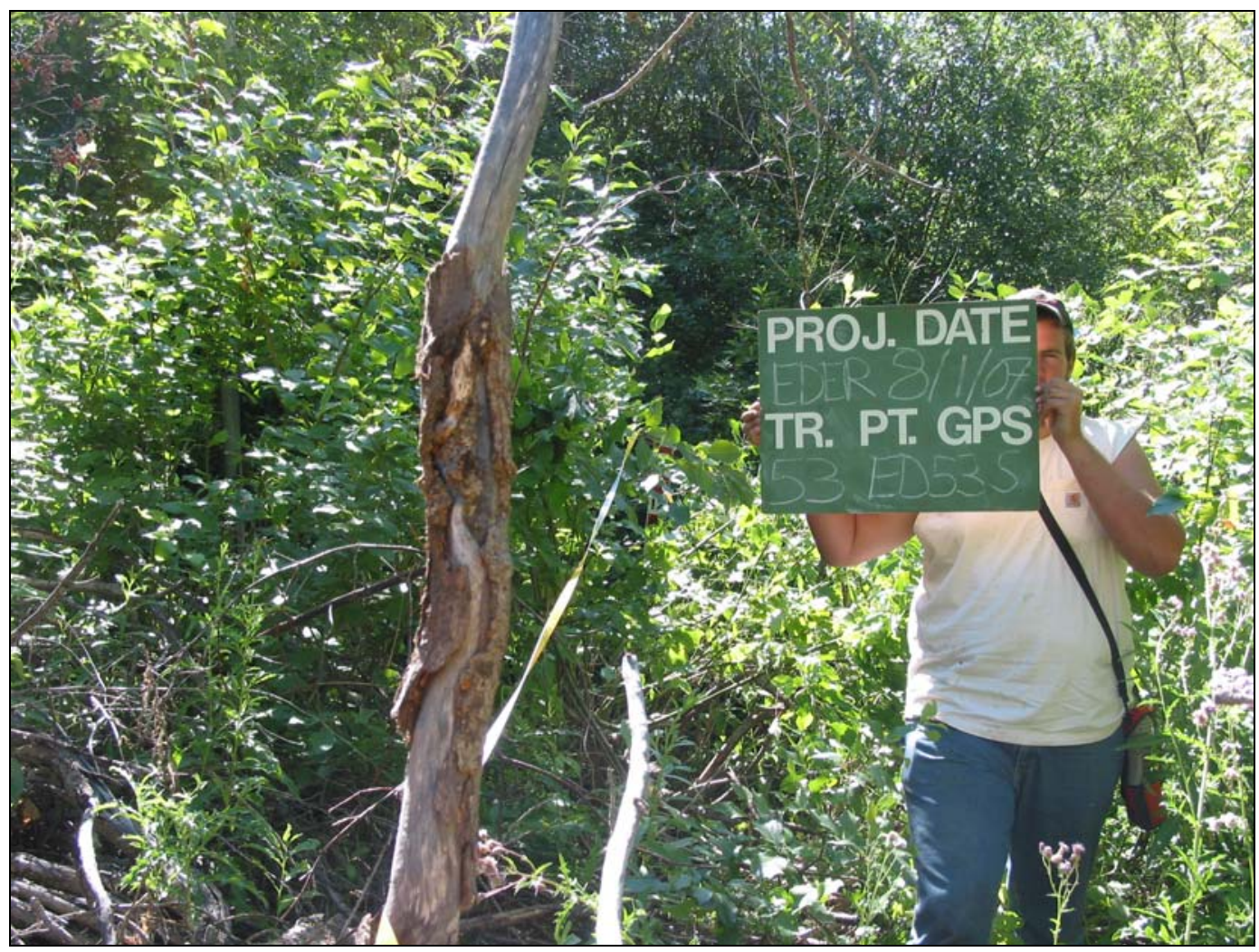

Transect 55

No Photograph 
Eder Acquisition 2007 HEP Report

\section{Transect 69}

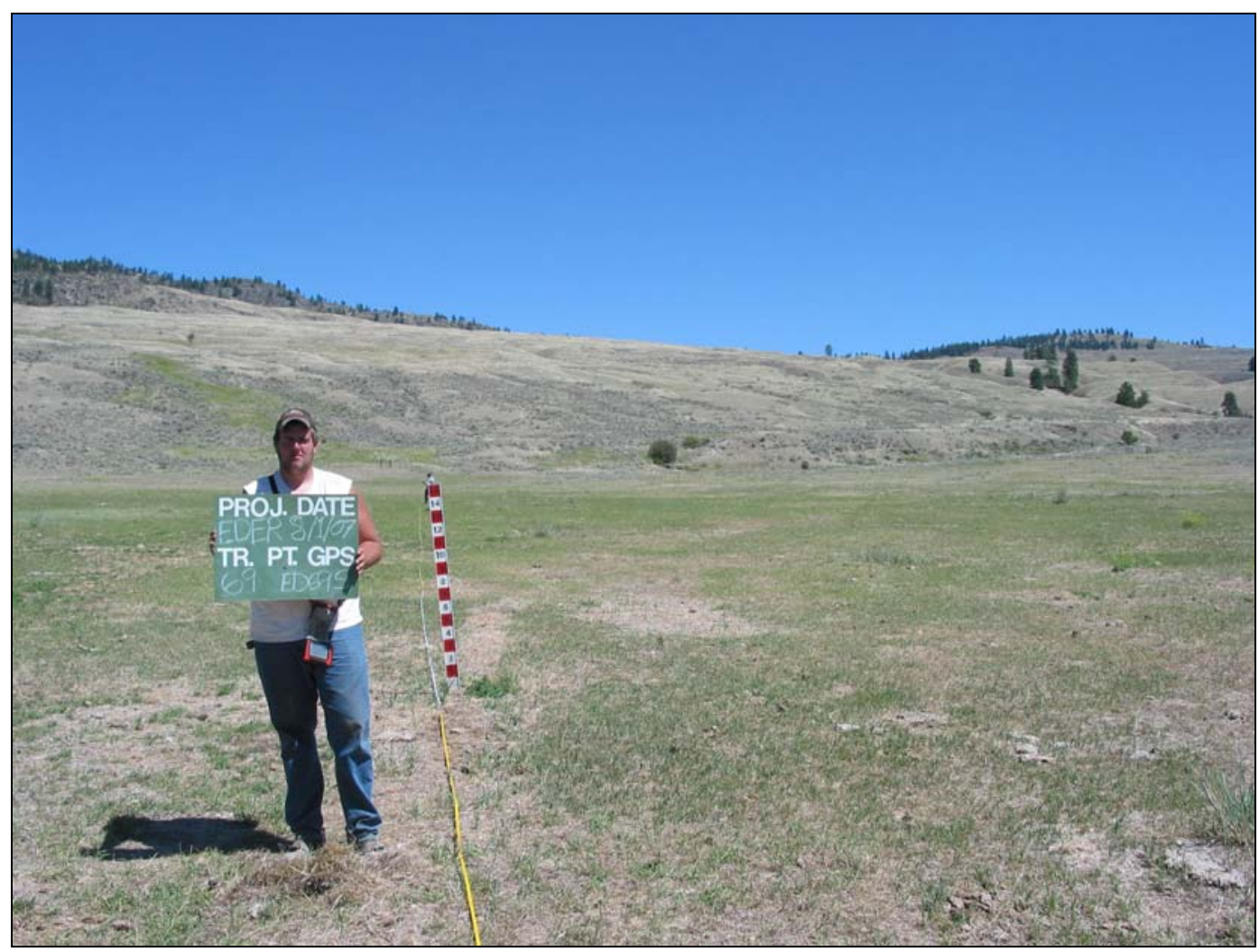

